\title{
Single-cell analysis of Foxp1-driven mechanisms essential for striatal development
}

Ashley G. Anderson ${ }^{1}$, Ashwinikumar Kulkarni ${ }^{1}$, Matthew Harper ${ }^{1}$, and Genevieve Konopka ${ }^{1 *}$

${ }^{1}$ Department of Neuroscience, UT Southwestern Medical Center, Dallas, TX, 75390-9111, USA

*Correspondence: Genevieve.Konopka@utsouthwestern.edu

Keywords: single-cell RNA-sequencing, striatum, neurodevelopment, FOXP1, autism

\section{Abstract}

The striatum is a critical forebrain structure for integrating cognitive, sensory, and motor information from diverse brain regions into meaningful behavioral output. However, the transcriptional mechanisms that underlie striatal development and organization at single-cell resolution remain unknown. Here, we show that Foxp1, a transcription factor strongly linked to autism and intellectual disability, regulates organizational features of striatal circuitry in a cell-type-dependent fashion. Using single-cell RNA-sequencing, we examine the cellular diversity of the early postnatal striatum and find that cell-type-specific deletion of Foxp1 in striatal projection neurons alters the cellular composition and neurochemical architecture of the striatum. Importantly, using this approach, we identify the noncell autonomous effects produced by disrupting Foxp1 in one cell-type and the molecular compensation that occurs in other populations. Finally, we identify Foxp1-regulated target genes within distinct cell-types and connect these molecular changes to functional and behavioral deficits relevant to phenotypes described in patients with FOXP1 loss-of-function mutations. These data reveal cell-type-specific transcriptional mechanisms underlying distinct features of striatal circuitry and identify Foxp1 as a key regulator of striatal development.

\section{Introduction}

The striatum is the major input nucleus of the basal ganglia and receives dense glutamatergic inputs from the cortex and thalamus, as well as dopaminergic innervations from the substantia nigra and other neuromodulatory circuits. The principal neurons that receive and integrate this information 
bioRxiv preprint doi: https://doi.org/10.1101/611780; this version posted April 18, 2019. The copyright holder for this preprint (which was not certified by peer review) is the author/funder, who has granted bioRxiv a license to display the preprint in perpetuity. It is made available under aCC-BY-ND 4.0 International license.

within the striatum are GABAergic spiny projection neurons (SPNs) ${ }^{1}$. Proper function of striatal circuitry is essential for coordinated motor control, action selection, and reward-based behaviors ${ }^{2,3}$. Dysfunction of this system is implicated across many neurological disorders, including Huntington's disease, Parkinson's disease, autism spectrum disorder (ASD), and obsessive-compulsive disorder ${ }^{4,5}$.

Striatal organization has two prominent features: the division of the striatum into distinct neurochemical zones, the striosome and matrix compartments, and the division of SPNs into the direct or indirect projection pathways. Striosome and matrix compartments are enriched for distinct neuropeptides and contribute differentially to striatal connectivity and behavior ${ }^{4,6-8}$. Recent evidence suggests that striosome-matrix compartmentalization is the initial organizational plan during striatal development with distinct intermediate progenitor pools in the lateral ganglionic eminence (LGE) giving rise first to striosome SPNs then matrix $\mathrm{SPNs}^{9}$. These progenitor pools then generate either direct and indirect pathway SPNs, which populate both compartments ${ }^{9}$. Direct pathway SPNs express dopamine receptor 1 (D1, dSPNs) and project to the globus pallidus internal (GPi) and substantia nigra (SN). Indirect pathway SPNs express dopamine receptor 2 (D2, iSPNs) and project to the globus pallidus external ${ }^{1}$. Ultimately, these pathways work to bidirectionally modulate excitatory inputs back onto the cortex ${ }^{1}$.

Mature dSPNs and iSPNs have distinct molecular profiles based on expression profiling studies $^{10-13}$, and several transcription factors and chromatin regulators have been identified for both pan-SPN and d/iSPN sub-specification ${ }^{14-26}$. However, a limitation of these previous studies was that non-cell autonomous changes in gene expression were unable to be detected. Moreover, the cellular diversity of the early postnatal striatum, in general, has not been characterized at single-cell resolution. This time point is an important and understudied period of striatal development before excitatory synaptic density onto SPNs markedly increases and where perturbations of cortical-striatal activity can have long lasting effects on SPN spine density and circuit activity ${ }^{27,28}$. 
Forkhead-box protein 1 (Foxp1) is a transcription factor with enriched expression in the striatum compared to the rest of the brain ${ }^{11}$. Foxp1 is highly expressed within both SPN populations and loss-of-function FOXP1 variants are strongly linked to ASD and intellectual disability in humans ${ }^{29-}$ 31. Expression of Foxp1 begins in the LGE at E12.5 with enrichment in the marginal zone and is maintained throughout striatal development ${ }^{26,32}$. While previous studies have suggested a role for Foxp1 in striatal development ${ }^{33,34,35}$, no study has examined the contribution of Foxp1 to striatal circuit organization in a cell-specific manner.

To ascertain the cell-type specific role of Foxp1, we generated mice with deletion of Foxp1 from dSPNs, iSPNs, or both populations, and used a combination of single-cell RNA-sequencing (scRNA-seq), whole brain 3D-imaging, and behavioral assays to delineate the contribution of Foxp1 to striatal development and function. We show that Foxp1 is crucial for maintaining the cellular composition of the striatum, especially iSPN specification, and proper formation of the striosomematrix compartments. We uncover downstream targets regulated by Foxp1 within iSPNs and dSPNs and connect these molecular findings to cell-type-specific deficits in motor and limbic systemassociated behaviors, including motor-learning, ultrasonic vocalizations, and fear conditioning. Moreover, we identify the non-cell autonomous effects produced by disruption of one SPN subpopulation and the molecular compensation that occurs. These findings provide an important molecular window into postnatal striatal development and further our understanding of striatal circuits mediating ASD-relevant behavioral phenotypes.

\section{Results}

\section{Early postnatal scRNA-seq of striatal cells across Foxp1 cKO mice}

To examine the contribution of Foxp1 to striatal development in a cell-type-specific manner, we generated Foxp1 conditional knockout (cKO) mice using BAC-transgenic mice driving Cre expression under the D1- or D2-receptor promoters ${ }^{36}{\text { crossed to Foxp } 1^{\text {floxflox }} \text { mice }}^{37-39}$ (Fig. 1a). Four genotypes were used for downstream analyses: Drd1-Cre ${ }^{\text {tg/+ }}$; Foxp1 ${ }^{\text {floxflox }}$ (Foxp1 ${ }^{D 1}$, deletion of Foxp1 in 
bioRxiv preprint doi: https://doi.org/10.1101/611780; this version posted April 18, 2019. The copyright holder for this preprint (which was not certified by peer review) is the author/funder, who has granted bioRxiv a license to display the preprint in perpetuity. It is made available under aCC-BY-ND 4.0 International license.

dSPNs), Drd2-Cre ${ }^{\operatorname{tg} /+}$; Foxp1 $1^{\text {floxfllox }}$ (Foxp1 ${ }^{D 2}$, deletion of Foxp1 in iSPNs), Drd1-Cre ${ }^{\operatorname{tg} /+}$; Drd2-Cre ${ }^{\operatorname{tg} /+}$; Foxp $1^{\text {floxfllox }}\left(\right.$ Foxp1 $^{\text {DD }}$, deletion of Foxp1 in both d/iSPNs), and Foxp ${ }^{\text {floxfllox }}$ (Foxp $1^{\text {CTL }}$ ). We confirmed that Foxp1 was reduced at both the transcript and protein levels within the striatum (Fig. 1b-d). Foxp1 is also reduced in lower-layer cortical neurons expressing Drd1 (Supplementary Fig. 1a).

Using 10X Genomics Chromium technology ${ }^{40}$, we profiled the transcriptome of 62,778 striatal cells across control and the three Foxp1 cKO mouse lines at postnatal day 9 ( $\mathrm{N}=4 /$ genotype; 16 samples total) (Fig. 1a). We detected 5,587 UMIs (median=3,837) and 1,794 genes (median=1,532) per cell (Fig. 1e). All cells were combined across genotype and filtered for downstream clustering, resulting in 43 clusters driven by cell-type (Fig. 1f and Supplementary Table 1). For unbiased characterization of striatal cell-types, we used a previously annotated adult striatal single-cell datase $^{13}$ to assign cell-types to each cluster using two separate methods, a previously published expression weighted cell-type enrichment (EWCE) analysis ${ }^{41}$ and an in-house correlation analysis (see methods) (Supplementary Fig. 1b). We confirmed the cell-type annotation of our dataset by examining the expression of known marker genes for each major cell-type (Supplementary Fig. 1c-f and Supplementary Table 1). The principal cell-types found within the early postnatal striatum were neurogenic progenitor cells, spiny projection neurons (SPNs), astrocytes, and oligodendrocyte precursor cells (OPCs) (Fig. 1f, g and Supplementary Table 2). Endothelial, microglia, ependymal, interneurons, and mural cells made up a smaller percentage of total cells within the postnatal striatum (Fig. 1f, g). Unexpectedly, at postnatal day 9, we found a large neurogenic progenitor population ( $30 \%$ of total cells within control samples) with clusters expressing proliferation markers (Mki67, Supplementary Fig. 1e), progenitor markers (Ascl1, D/x2, Supplementary Fig. 1e), and SPNspecification markers (Sp9, Ppp1r1b, Drd1, Drd2, Supplementary Fig. 1f). These data suggest ongoing striatal neuronal differentiation and neurogenesis into early postnatal development.

The cell-type with the largest number of unique subclusters were SPNs with 13 unique clusters (Fig. 1f). SPNs and neurogenic progenitors made up 52\% of the total cell population (Fig. 19) in line with previously published adult scRNA-seq datasets ${ }^{12,13}$. Genotype-specific variations were observed 
primarily within SPN clusters, where Foxp1 is selectively deleted (Fig. 19-j and Supplementary

Table 2). To more directly compare the composition of striatal cell-types across genotypes and better control for variations in total cells sequenced between genotypes, we down-sampled the dataset to yield equal cell numbers across genotypes and reclustered the resultant cells separately. We found analogous results in the percentage of cell-types from down-sampling experiments compared to the full dataset (Supplementary Fig. 1g). Variations within the SPN and progenitor populations in Foxp1 cKO samples compared to control were consistent across down-sampling, with more neurons (SPNs) and fewer progenitor cells within all Foxp1 cKO samples (Fig. 1g-j, Supplementary Fig. 1g, and Supplementary Table 2). Our data reveal at the single-cell level that deletion of Foxp1 reduces the population of striatal neurogenic cells, while increasing the percentage of mature SPNs. These data highlight the diversity of the cellular composition of the early-postnatal striatum and demonstrate that Foxp1 plays an important role in striatal neurogenesis and development.

\section{Diversity of early post-natal striatal projection neurons}

To further characterize early postnatal SPN subtypes and the effects of Foxp1 deletion, we next isolated all clusters identified as neuronal from the annotation analyses (see Figure 1 and methods) and reclustered them separately (18,073 cells total and 24 clusters) (Fig. 2a-e). Three interneuron clusters (Clusters-14, 15, 20) were identified by the interneuron marker Nkx2-1 (Fig. 2a,

Supplementary Fig. 2a, and Supplementary Table 1). We could clearly distinguish dSPN clusters (Clusters-0, 1, 3, 4, 5, 9) and iSPN clusters (Clusters-2, 8, 10, 16) using canonical markers (Drd1 and Tac1 for dSPNs, Drd2 and Penk for iSPNs) (Fig. 2f, Supplementary Fig. 2b, and Supplementary Table 1). Pairwise comparisons between the major dSPN and iSPN clusters confirmed enrichment of known genes within each population (Supplementary Fig. 2c). One small cluster (Cluster-19) coexpressed both Drd1 and Drd2 receptors, termed "ddSPNs" (Fig. 2f). SPNs expressing both Drd1 and Drd2 receptors were also scattered throughout other clusters and comprised $\sim 1 \%$ of the total SPN population (Supplementary Fig. 2d and Supplementary Table 2). We identified a recently 
bioRxiv preprint doi: https://doi.org/10.1101/611780; this version posted April 18, 2019. The copyright holder for this preprint (which was not certified by peer review) is the author/funder, who has granted bioRxiv a license to display the preprint in perpetuity. It is made available under aCC-BY-ND 4.0 International license.

described SPN subpopulation termed "eccentric" SPNs (eSPNs) ${ }^{13}$ within Cluster-7 that uniquely expressed markers such as Casz1 and Otof (Fig. 2f, Supplementary Fig. 2e, and Supplementary Table 1). We also found two clusters (Cluster-6, 23) that were enriched for the neurogenic transcription factors Sox4 (Fig. 2f and Supplementary Fig. 2f) and Sox11 (Supplementary Table 1). Sox4 and Sox11 function during the terminal steps of neurogenesis to promote neuronal maturation ${ }^{42,43}$; therefore, we termed these clusters "immature SPNs" (imSPNs). We confirmed the presence of Sox $4^{+}$cells within and near the subventricular zone of the lateral ventricle and populating zones in P7 ventral striatum (Supplementary Fig. 2g). Additionally, several clusters enriched for d/iSPN markers also have high expression of Sox4 (dSPN Clusters-9,11, 13, 17 and iSPN Clusters16), indicating these may be less mature SPNs (Fig. $\mathbf{2 g}$ and Supplementary Fig. 2f). Two clusters (Cluster-12, 18) were composed primarily of cells from Foxp $1^{D 1}$ and Foxp $1^{D 2}$ and could not be classified into distinct SPN subclusters (Fig. 2a-f). Foxp2, another Foxp transcription factor with high amino acid sequence similarity to Foxp $1^{44}$, is an SPN marker with enriched expression in dSPNs

(Supplementary Fig. 2c) ${ }^{29,45}$. Within our dataset, Foxp2 is highly expressed within all dSPN clusters and one iSPN cluster (Cluster-8). Surprisingly, the highest expression of Foxp2 is found within eSPN Cluster-7 and imSPN Cluster-6, where notably Foxp1 is not highly expressed (Fig. 2f and Supplementary Fig. 2h). Foxp2 expression is also maintained within adult eSPNs ${ }^{13}$. We confirmed that Foxp2 is expressed in cells other than mature dSPNs and iSPNs at postnatal day 9 using D1tdTomato ${ }^{\text {tg/- }}$ and D2-eGFP ${ }^{\text {tg/- }}$ reporter mice (Supplementary Fig. 2i).

\section{Foxp1 regulates SPN subtype composition and iSPN specification}

We next asked whether Foxp1 regulates the development of specific SPN populations by examining the percentages of SPN subtypes across genotypes (Fig. $\mathbf{2 g}$ ). Control samples have nearly double the number of dSPN relative to iSPNs $(61 \%$ dSPNs, $31 \%$ iSPNs), with imSPNs contributing $\sim 4 \%$ of the total SPN population and both eSPNs and ddSPNs contributing $\sim 2 \%$ (Fig. $\mathbf{2 g}$ ). This percentage of dSPNs to iSPNs at P9 is similar to those seen at P14 using reporter mice ${ }^{46}$. 
The percentage of SPN subtypes varied across Foxp1 cKO samples (Fig. $\mathbf{2 g}$ ). Notably, the number of eSPNs increased 2-4-fold across Foxp1 CKO samples. Strikingly, within Foxp $1^{D 2}$ and Foxp $1^{D D}$ samples, the number of iSPNs was reduced by two-thirds compared to control levels (Fig. 2g). Cells with deletion of Foxp1 were transcriptionally distinct and clustered largely separately from control cells (Fig. 2h).

To independently confirm the reduction of iSPNs in Foxp $1^{D 2}$ and Foxp $1^{D D}$ samples, we crossed all Foxp1 cKO mice to D2-eGFP reporter animals (D2-eGFP'tg/; Foxp $1^{\text {floxflox }}$ ) to label iSPNs (Fig. 2i). Within Foxp $1^{D 2}$ mice, we again found a significant two-thirds reduction of iSPNs (D2-eGFP ${ }^{+}$cells) as seen in the scRNA-seq data (Fig. 2i, j). Compared to Foxp $1^{C T L}$, Foxp $1^{D D}$ mice also showed significantly reduced iSPNs, but they also showed increased iSPNs compared to Foxp $1^{D 2}$ (Fig. 2i, j). The remaining iSPNs in the Foxp1 $1^{D 2}$ mice were not the product of D2-Cre inefficiency, as these cells did not express Foxp1 (Supplementary Fig. 2j). Only 7 iSPNs within the single-cell Foxp ${ }^{\text {CTL }}$ data did not express Foxp1 (0.2\% of total iSPNs) (Supplementary Fig. 2d), therefore, we would not expect the remaining iSPNs in Foxp $1^{D 2}$ mice to be a naturally occurring Foxp1 negative population. Taken together, these results indicate that Foxp1 is required for the specification of distinct iSPN subpopulations and may function to repress the generation of eSPNs.

\section{Deletion of Foxp1 disrupts striosomal area and iSPN localization}

We identified distinct subclusters within dSPNs and iSPNs in our scRNA-seq data (Fig. 3a). Using a pairwise differential gene expression analysis between clusters, we found that two Foxp $1^{\text {CTL }}$ dSPN clusters (Cluster-0,5) corresponded to either matrix or striosome compartments, respectively, based on the enrichment of known striosome (Oprm1, Is/1, Pdyn, Lypd1, Tac1, and Nnat) or matrix markers (Ebf1, Epha4, Mef2c) (Fig. 3b and Supplementary Table 3). Within iSPNs, Cluster-8 was enriched for striosomal markers (Nnat, Lypd1, Foxp2) and Cluster-2 for matrix markers (Penk, Chrm3, Epha4) (Fig. 3c and Supplementary Table 3). 
We next wanted to determine whether the remaining subpopulation of iSPNs within Foxp $1^{D 2}$ or Foxp $1^{D D}$ mice localized within either the striosome or matrix compartment. To do this, we stained for the canonical striosome marker MOR (Oprm1) in Foxp1 cKO mice crossed to D2-eGFP reporter mice (Fig. 3d-f). We found that few remaining iSPNs within Foxp $1^{D 2}$ and Foxp $1^{D D}$ mice localized within the striosome compartment. They clustered primarily around the border of the striosome compartments and were scattered throughout the matrix (Fig. 3d-f). Taken together, these data show that Foxp1 specifies iSPNs of both striosome and matrix compartments but may not be necessary for specification of a subpopulation of iSPNs near the striosomal border. We also found that striosomal area was significantly reduced across all Foxp1 cKO animals at P7 (Fig. $\mathbf{3 g}$ ) and that fewer striosome "patches" were observed specifically within Foxp $1^{D D}$ mice (Fig. 3h). These data indicate that Foxp1 plays a critical role within both dSPNs and iSPNs to maintain proper striosome-matrix architecture.

\section{Cell-type-specific Foxp1 regulated targets}

To better understand the molecular mechanisms regulated by Foxp1, we performed a celltype-specific "pseudobulk" differential gene expression analysis (see methods) of the scRNA-seq data across genotypes. We identified differentially expressed genes (DEGs) regulated by Foxp1 within dSPNs or iSPNs, both cell-autonomously and non-cell-autonomously (Fig. $\mathbf{4 a ,}$ b and Supplementary Table 4). Cell-autonomous DEGs are found in Cre active cells (dSPNs in Foxp $1^{D 1}$ samples or iSPNs in Foxp $1^{D 2}$ samples) and non-cell-autonomous DEGs are found in Cre inactive cells (iSPNs in Foxp $1^{D 1}$ samples or dSPNs in Foxp $1^{D 2}$ samples). We observed more total iSPN-DEGs (647) compared to dSPNs-DEGs (285) across genotypes (Fig. 4a, b). There were more cellautonomous changes than non-cell-autonomous within both dSPNs and iSPNs of Foxp $1^{D 1}$ and Foxp $1^{D 2}$ samples and no differences in the ratio of cell-autonomous to non-cell autonomous DEGs within dSPNs or iSPNs were observed (Fig. 4c). However, significantly more iSPN-DEGs were shared between Foxp $1^{D 2}$ and Foxp $1^{D D}$ samples (211 DEGs) compared to dSPN-DEGs shared between Foxp $1^{D 1}$ and Foxp $1^{D D}$ samples (47 DEGs) (Fig. 4d). The DEGs unique to Foxp $1^{D D}$ samples 
were termed "interaction-DEGs". We found significantly more interaction-DEGs in dSPNs suggesting that iSPN dysfunction exerts more transcriptional changes upon dSPNs than vice versa (Fig. 4 d).

The striking difference in total number of DEGs between iSPNs and dSPNs could be due to transcriptional compensation by Foxp2 in dSPNs. Foxp2 is enriched in dSPNs relative to iSPNs (Supplementary Fig. 2c) and we previously found that Foxp1 and Foxp2 have shared striatal targets $^{35}$. Interestingly, Foxp2 is increased in iSPNs with loss of Foxp1, suggesting that Foxp1 may function to repress Foxp2 within distinct iSPN subtypes (Fig. 4a and Supplementary Table 4). Six3 (Six homeobox 3), a transcription factor crucial for iSPN specification ${ }^{26}$, is also upregulated within the remaining iSPNs of $F o x p 1^{D 2}$ and Foxp $1^{D D}$ mice (Supplementary Table 4). We previously found that SIX3 was a direct target of FOXP1 in human neural progenitors ${ }^{35}$. Therefore, upregulation of both Foxp2 and Six3 in iSPNs may play a role in the specification of the remaining iSPNs within Foxp1 ${ }^{D 2}$ and Foxp $1^{D D}$ mice.

Gene ontology (GO) analysis of the shared iSPN-DEGs within Foxp $1^{D 2}$ and Foxp $1^{D D}$ supports a role for Foxp1 in axon guidance, neurogenesis, and neuronal differentiation of iSPNs (Supplementary Table 5). Shared upregulated dSPN-DEGs within Foxp $1^{D 1}$ and Foxp $1^{D D}$ suggest altered synaptic and voltage-gated mechanisms (Supplementary Table 5). We confirmed changes in cell-type-specific gene expression via immunohistochemistry for a subset of top DEGs (Pde1a, Calb1, and Darpp32) using dual-reporter mice labelling dSPNs with tdTomato (Drd1-tdTomato ${ }^{\text {tg/t}}$;

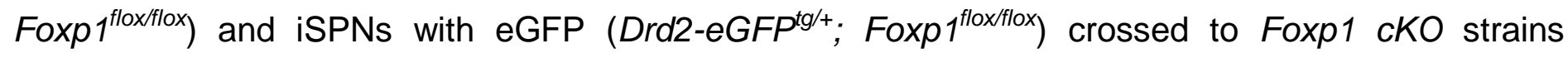
(Supplementary Fig. 3a-e). Pde1a, a gene encoding a calmodulin $/ \mathrm{Ca}^{2+}$ activated phosphodiesterase, was upregulated in both SPN subtypes within all Foxp1 cKO samples in a cell autonomous and non-cell-autonomous manner (Fig. 4a, b and Supplementary Fig. 3a, d-e). Previous in vitro work found that loss of Foxp1 reduced the expression of DARPP-32 (Ppp1r1b), a critical phosphatase in the dopamine signaling cascade ${ }^{33}$. We show this decrease in DARPP-32 is specific to iSPNs in vivo (Supplementary Fig. 3b, d-e). We also confirmed the increase of calbindin 1 (Calb1) selectively in dSPNs with deletion of Foxp1 (Supplementary Fig. 3c-e). 
Given our previous finding that striatal targets of Foxp1 overlapped significantly with ASDassociated genes ${ }^{35}$, we examined the cell-type-specificity of this overlap (Supplementary Fig. $\mathbf{3 f}$ ). Using the SFARI ASD gene list, we found a significant overlap with high-confidence ASD-risk genes (SFARI gene score 1-4) with iSPNs-DEGs with cell-autonomous deletion of Foxp1. These genes included three members of the contactin-family of axon-associated cell adhesion molecules: Cntn4, Cntn5, Cntn6 (Fig. 4a, Supplementary Fig. 3f). There was no significant overlap with ASD-risk genes and cell-autonomous DEGs in dSPNs (Supplementary Fig. 3f). Surprisingly, we found a significant overlap with upregulated, non-cell autonomous iSPN-DEGs within Foxp ${ }^{D 1}$ samples (Kirrel3, Nlgn1) (Supplementary Fig. 3f). Both iSPN- and dSPN-DEGs within Foxp1 ${ }^{D D}$ samples overlapped with ASD-risk genes (Supplementary Fig. 3f). These data demonstrate that cell-typespecific deletion of Foxp1 specifically within iSPNs modulates ASD-associated molecular pathways both cell-autonomously and non-cell-autonomously.

Two ASD-risk genes that were upregulated with deletion of Foxp1 in dSPNs were Cntnap2 (contactin-associated protein like 2) and Dpp10 (dipeptidyl peptidase like 10) (Fig. 4b and Supplementary Table 4). Cntnap2 is a known repressed downstream target of both Foxp1 and Foxp2 ${ }^{47,48}$ and we previously found upregulation of Dpp10 within Foxp1 ${ }^{+/}$striatal tissue using bulk RNA-sequencing ${ }^{35}$. Here, using scRNA-seq, we show this regulation is specific to dSPNs.

\section{Upregulation of eSPN molecular markers with deletion of Foxp1}

To determine whether deletion of Foxp1 within SPNs altered cell identity, we overlapped the top 50 enriched gene markers of distinct SPN subpopulations (eSPNs, imSPNs, and matrix and striosome dSPNs and iSPNs) (Supplementary Table 1) with upregulated or downregulated iSPNDEGs (Fig. 4e) or dSPN-DEGs (Fig. 4f) found within each Foxp1 cKO group. The upregulated DEGs in both iSPNs and dSPNs with cell-autonomous deletion of Foxp1 were significantly enriched for molecular markers of eSPNs. Upregulated iSPN-DEGs were specifically enriched for the top four enriched eSPNs markers: Adarb2, Ntng1, Asic2, and Foxp2 (Fig. 4a, e-f). iSPN and dSPN subtype 
enriched genes significantly overlapped with downregulated DEGs in both Foxp $1^{D 1}$ and Foxp $1^{D 2}$ samples (Fig. 4a, e-f). Taken together, these results indicate that Foxp1 is important for maintaining the molecular identity of dSPNs and iSPNs within both matrix and striosome compartments and repressing eSPN molecular identity within these cell-types.

\section{Altered direct and indirect pathway projections in Foxp $1^{D 2}$ mice}

Many DEGs regulated by Foxp1 within SPNs are involved in axonogenesis and neuron projection (Supplementary Tables 4 and 5). We therefore examined SPN projection patterns impacted by celltype-specific deletion of Foxp1 in adult mice using serial two-photon tomography combined with a machine-learning-based quantification algorithm ${ }^{49,50}$. We crossed Foxp1 ${ }^{D 1}$ and Foxp1 ${ }^{D 2}$ mice to D1tdTomato and/or D2eGFP reporter mice (described above) to visualize projection patterns of both the direct (dSPN) and indirect (iSPN) pathway, respectively. We first quantified total striatal area across genotypes and found a significant decrease in striatal area in Foxp $1^{D 2}$ mice, while no changes were found in Foxp $1^{D 1}$ animals (Fig. 5a). We next found a significant reduction of iSPN terminals onto the GPe in Foxp $1^{D 2}$ mice, which was not unexpected given the significant decrease in iSPNs (Fig. $5 \mathbf{b}$, de). iSPN terminals onto the GPe were unaltered in Foxp $1^{D 1}$ mice (Fig. 5 b-c, e). Moreover, there were no changes in dSPN projection patterns in Foxp1 ${ }^{D 1}$ mice; however, Foxp $1^{D 2}$ mice had significant deficits in dSPN projections onto the GPi, supporting a non-cell-autonomous role for Foxp1 in iSPNs (Fig.5b-d, f). These findings indicate that Foxp1 regulates both iSPN and dSPN projection patterns through its role in iSPNs (Fig. $\mathbf{5 g}$ ). Within our scRNA-seq data, non-cell-autonomous dSPN-DEGs in Foxp $1^{D 2}$ samples were enriched for GO categories such as neuron projection (Supplementary Table 5). Since projections onto the GPi were not altered in Foxp $1^{D 1}$ mice, dSPN-DEGs unique to Foxp $1^{D 2}$ samples are most likely responsible for the altered dSPN projection patterns found within Foxp $1^{D 2}$ animals. We therefore examined the overlap of dSPN-DEGs within Foxp1 ${ }^{D 1}$ (cell-autonomous) and Foxp $1^{D 2}$ samples (non-cell-autonomous) (Fig. 5h). dSPN-DEGs unique to Foxp $1^{D 2}$ samples involved in neuron projection include Akap5, Asic2, Kirre/3, Cdh8, and Cntn4 (Fig. 5h). Interestingly, Kirre/3, 
Cdh8, and Cntn4 are also ASD-risk genes (Fig. 5h). These findings suggest deletion of Foxp1 within

iSPNs alters the gene expression profiles within both iSPNs and dSPNs important for proper striatal projection patterning.

\section{Distinct behavioral deficits with cell-type-specific deletion of Foxp1}

We hypothesized that severe reduction of iSPNs and altered projection patterns with deletion of Foxp1 from iSPNs would result in altered motor behaviors. We therefore first tested behaviors classically characterized as being governed by striatal circuits, such as motor learning and activity levels. To test motor learning, we used the accelerating rotarod assay and found that Foxp $1^{D 2}$ and Foxp $1^{D D}$ mice had significant deficits at remaining on the accelerating beam compared to control and Foxp $1^{D 1}$ mice (Fig. 6a). This phenotype was not due to differences in grip strength (Supplementary Fig. 4a, b) or gait abnormalities (Supplementary Fig. 4c-f). Foxp $1^{D 2}$ and $F o x p 1^{D D}$ mice were also hyperactive in the open field behavioral paradigm compared to control mice (Fig. 6b); however, no difference was observed in novel cage activity between genotypes (Supplementary Fig. $\mathbf{4 g}$ ). There was no difference in time spent in the periphery versus the center of the open field between genotypes (Fig. 6c), suggesting no changes in anxiety-like behavior.

Since genetic variants in FOXP1 are strongly associated with ASD, we next examined ASDrelevant social communication behaviors. Using a maternal separation paradigm, we recorded pup ultrasonic vocalizations (USVs) at three postnatal time points (P4, P7, and P10). We found that Foxp $1^{D 1}$ mice produced significantly fewer calls with altered call slope compared to control pups (Fig. 6d-e). In addition, Foxp $1^{D 1}$ pups had significantly lower pitch at P4, while Foxp $1^{D D}$ mice exhibited deficits in pitch across all developmental time points (Fig. 6f). No significant USV changes were measured solely in Foxp $1^{D 2}$ pups. We also tested nest building behavior, an important communal behavior in rodents ${ }^{51,52}$, and found that $F o x p 1^{D 1}$ and Foxp $1^{D D}$ mice produced low-quality nests compared to control and Foxp2 ${ }^{D 2}$ nests (Fig. $\mathbf{6 g}-\mathbf{h}$ ). 
bioRxiv preprint doi: https://doi.org/101101/611780; this version posted April 18,2019 . The copyright holder for this preprint (which was not certified by peer review) is the author/funder, who has granted bioRxiv a license to display the preprint in perpetuity. It is made available under aCC-BY-ND 4.0 International license.

Because individuals with FOXP1 mutations are frequently comorbid for intellectual disability $^{30,31}$, we next assessed whether learning and memory circuits were altered using the cued and contextual fear conditioning (FC) paradigm (Fig. 6i-j). All Foxp1 cKO mice had significantly reduced freezing behavior during cued-evoked fear memory recall (Fig. 6i); however, only Foxp1 ${ }^{D 1}$ and Foxp $1^{D D}$ mice showed significant deficits in context-evoked fear memory (Fig. 6j). While hippocampal and amygdala circuits are classically associated with fear conditioning, striatal D1 receptors are also important for mediating proper contextual FC in mice ${ }^{53}$. We also found that striosome-matrix architecture was more severely disrupted over postnatal development in Foxp $1^{D 1}$ and Foxp $1^{D D}$ adult animals compared to control and Foxp $1^{D 2}$ mice (Supplementary Fig. 4h).

\section{Discussion}

In this study, we use single-cell transcriptomics to examine the molecular mechanisms underlying striatal neuronal specification by sequencing thousands of striatal cells across control and cell-type-specific Foxp1 conditional mouse models. We show that Foxp1 influences striatal development through cell-type-specific molecular pathways and describe the molecular, functional, and behavioral consequences of Foxp1 deletion within distinct striatal circuits (Fig. 7).

The first weeks of postnatal striatal development is an important period of excitatory synaptogenesis onto SPNs ${ }^{27,28,54}$ and the cellular composition of the striatum during this time has been understudied. We surprisingly found that neurogenic progenitors make up a large component of the early postnatal striatum and that deletion of Foxp1 decreases the ratio of these neurogenic progenitors to mature SPNs. These findings suggest that Foxp1 regulates intermediate progenitor pools and the differentiation of SPNs within the developing striatum. Furthermore, we found that Foxp1 is required for the specification of iSPNs that localize to the matrix and striosome compartments. iSPNs that remain with deletion of Foxp1 localize to the striosome-matrix border and significantly upregulate top marker genes of a recently identified eSPN population, including Foxp2 ${ }^{13}$. Future work will help resolve the functional contribution of eSPNs to striatal development. 
bioRxiv preprint doi: https://doi.org/10.1101/611780; this version posted April 18,2019 . The copyright holder for this preprint (which was not

certified by peer review) is the author/funder, who has granted bioRxiv a license to display the preprint in perpetuity. It is made available under aCC-BY-ND 4.0 International license.

Deletion of Foxp1 specifically within iSPNs leads to both cell-autonomous and non-cellautonomous changes in SPN projection patterns. Fewer iSPN terminals onto the globus pallidus external and fewer dSPN terminals onto the globus pallidus internal were observed. dSPNs and iSPNs are known to form inhibitory axon collaterals onto neighboring SPNs and modulate their excitability ${ }^{55,56,57}$. iSPNs and dSPNs also cooperate together to intermix within the striosome and matrix compartments ${ }^{58}$. We not only found that manipulation of iSPNs led to functional changes of dSPNs, but we captured a molecular snapshot of this inter-SPN communication, including differentially expressed ASD-risk genes involved in neuron projection such as Cntn4, Cdh8, and Kirre/3.

FOXP1 is among a subset of genes repeatedly and significantly linked to $A S D^{59,60}$. Thus, connecting our molecular findings to behavioral deficits is particularly relevant to a behaviorally diagnosed disorder that hinges upon two key behavioral phenotypes, impairments in language and social interactions and restrictive or repetitive behaviors. The majority of individuals with FOXP1 mutations are diagnosed with ASD and all reported cases are comorbid with intellectual disability, gross motor delays, and/or selective language impairments ${ }^{30,31}$. We found that mice with iSPNdeletion of Foxp1 caused significant motor disruptions, as measured by increased hyperactivity and motor-learning deficits on the rotarod. Concordant with our data, mice with ablated iSPNs or mice with Darpp32 deletion from iSPNs were also hyperactive in the open field ${ }^{61,62}$. Adult mice with induced ablation of D2-receptors displayed severe motor learning impairments on the accelerating rotarod $^{63}$. These data indicate that loss of iSPNs with deletion of Foxp1 lead to significant motorlearning and activity deficits.

Pup USVs are an important measure of affective state and social behavior in mice ${ }^{51,64}$ and peak between postnatal days 4 and $10^{35}$. Disruption of neonatal call number and structure with deletion of Foxp1 within dSPNs is particularly interesting given the high co-expression of both Foxp1 and Foxp2 within this cell-type and the ability of Foxp1 and Foxp2 to heterodimerize to regulate gene expression $^{65}$. Foxp2 plays a critical role in the vocal behavior across many species, including 
humans, mice and songbirds ${ }^{66}$. We show that Cntnap2, a known shared target of Foxp1 and Foxp2 ${ }^{47,48}$, is significantly upregulated within dSPNs. Variants in CNTNAP2 are also associated with ASD and Cntnap2 KO mice have altered pup USVs ${ }^{48}$. We previously found that Foxp1 heterozygous mice display altered USV phenotypes, including deficits in call number, call structure, and pitch ${ }^{35}$. Additionally, mice with cortical and hippocampal deletion of Foxp1 also produced fewer USVs, though no changes were observed in call structure or pitch $^{39}$. Here, we observed changes in all three parameters within Foxp $1^{D 1}$ and Foxp $1^{D D}$ mice suggesting that Foxp1 regulates distinct aspects of mouse vocal behavior largely through cortical-striatonigral circuitry.

Striosome compartments are smaller and architecturally disorganized with deletion of Foxp1 in iSPNs and/or dSPNs in the early postnatal striatum. Loss of striosome-matrix compartmentalization is particularly striking in adulthood with dSPN-specific deletion of Foxp1. These findings indicate that dSPN-targets regulated by Foxp1 exert a stronger influence over maintaining striatal neurochemical organization. Behaviors specific to Foxp $1^{D 1}$ mice include deficits in contextual fear memory recall, a known limbic-circuitry associated behavior. Striosomes receive preferential inputs from limbic subcortical regions, including the amygdala and bed nucleus of the stria terminalis ${ }^{8}$; thus, inputs from these limbic regions targeting striosomes may be disrupted and contribute to the limbic-associated behavioral deficits seen in Foxp $1^{D 1}$ and Foxp1 ${ }^{D D}$ mice. Additionally, mice with cortical and hippocampal deletion of Foxp1 did not show deficits in cued or contextual fear conditioning ${ }^{38}$. Therefore, Foxp1 is likely mediating fear conditioned behaviors via disruption of striatal circuits.

While ASD is a genetically complex disorder, several studies have shown that striatal SPNs may be particularly vulnerable to ASD-linked mutations ${ }^{67-71}$. Our study uncovers the molecular targets of Foxp1 in SPN subtypes and finds that Foxp1 regulates ASD-relevant behaviors via distinct striatal circuits. We show that iSPNs are particularly vulnerable with loss of Foxp1 and that Foxp1 regulated iSPN-targets are enriched for high-confidence ASD risk-genes, suggesting that striatopallidal circuitry might be particularly at risk with loss-of-function FOXP1 mutations. Our data provide important molecular insights for the development of future therapies targeting striatal circuits. 


\section{Figure Legends}

Fig. 1. Early postnatal scRNA-seq of striatal cells across Foxp1 cKOs. a) Schematic of the scRNA-seq experiment using striatal tissue from P9 mice ( $\mathrm{N}=4 /$ genotype) with cell-type-specific conditional deletion of Foxp1 within the dopamine receptor-1 $\left(F o x p 1^{D 1}\right)$, dopamine receptor-2 $\left(F_{o x p 1}{ }^{D 2}\right)$, or both $\left(F_{o x p}{ }^{D D}\right)$ cell-types. b-d) Foxp1 is reduced in the striatum via immunohistochemistry (P56) (b) and quantitative RT-PCR (P7) (c) within each cKO line, with near complete reduction in Foxp1 $1^{D D}$ striatal tissue via immunoblot (P56) (d) (100um scale bar). e) Violin plots of median and mean number of UMls or genes per cell across all genotypes. f) Non-linear dimensionality reduction with UMAP of all 62,778 post-filtered cells combined across genotype and used for downstream analyses. Cell-type annotation is overlaid to identify the major cell-type represented by each cluster (43 total clusters). $\mathbf{g}$-j) UMAP plot of cells from (f) color-coded to identify each cell by genotype. Pie charts using colors from (f) show the striatal cell-type composition as a percentage of total cells within each genotype.

Fig. 2. Foxp1 specifies distinct SPN subpopulations. a) UMAP plot showing each neuronal subcluster by color with overlay colors showing neuronal subpopulation identity. b-e) UMAP plots of cells from (a) color-coded to identify each cell by genotype. f) Violin plots of the normalized UMI expression of markers of SPN subpopulations: dSPNs (Drd1, Tac1, Foxp2), iSPNs (Drd2, Penk), ddSPNs (Drd2, Drd1, Tac1), eSPNs (Casz1), and imSPNs (Sox4). g) Pie charts showing altered composition of SPN subtypes within Foxp1 $c K O$ mice (using colors from a). h) Heatmap showing the percentage of cells contributing to each cluster across genotype (using colors from a). i-j) Foxp1 cKO mice were crossed to $D 2^{\text {EGFP }}$ reporter lines to label dopamine receptor-2 (D2R) iSPNs in green (coronal section, $500 \mu \mathrm{m}$ scale bar). Foxp $1^{D 2}$ and Foxp1 ${ }^{D D}$ mice had significantly fewer iSPNs 
compared to Foxp $1^{C T L}$ mice at P7, while Foxp $1^{D D}$ mice had significantly more iSPNs compared to Foxp $1^{D 2}$ animals. Data are represented as a box plot, $\mathrm{n}=3-6$ mice/genotype. ${ }^{* * *} \mathrm{p}<0.0001$, *** $<0.005$, one-way ANOVA with Tukey's multiple comparisons test.

Fig. 3. Foxp1 regulates striosome-matrix organization. a) Within Foxp $1^{C T L}$ samples, dSPN and iSPNs have large sub-clusters (Clusters- 0 and -5 for dSPNs and Clusters- 2 and -8 for iSPNs). Cells with deletion of Foxp1 cluster largely separately from control cells and subclusters within iSPNs and dSPNs are more intermixed (Foxp $1^{D 1}$ dSPNs) or lost completely (Foxp $1^{D 2}$ iSPNs). b-c) Scatter plots showing the percent expression of enriched transcripts between Clusters- 0 and-5 (b) or Clusters-2 and-8 (c). Striosome markers are enriched in dSPN Cluster-5 and iSPN Cluster-8, while matrix markers are enriched in dSPN Cluster-0 and iSPN Cluster-2 (p.adj<0.05). d-f) iSPNs within Foxp1 $1^{D 2}$ and Foxp $1^{D D}$ mice localized primarily along the striosomal border marked by $\mathrm{IHC}$ for Mu-Opiod Receptor (MOR) in P7 animals crossed to D2-eGFP reporter mice $(500 \mu \mathrm{m}$ scale bar in D-E, $100 \mu \mathrm{m}$ scale bar in $\mathbf{f})$. $\mathbf{g}$-h) The striosome compartment was significantly reduced across all Foxp1 cKO mice as a percent of total striatal area (measuring only dorsal striosomes) and the number of striosome "patches" was significantly reduced in Foxp $1^{D D}$ animals. Data are represented as mean \pm SEM, $\mathrm{n}=4$ mice/genotype. ${ }^{*} p<0.05, p^{* *}<0.005,{ }^{* \star *} p<0.0001$, one-way ANOVA with Tukey's multiple comparisons test.

Fig. 4. Foxp1 regulates cell-type-specific molecular pathways. a-b) SPN cell-type-specific differential gene expression between genotypes. Upset plot showing the overlap of upregulated or downregulated DEGs across genotypes within iSPNs (a) or dSPNs (b). Genes shown within boxes are color-coded by categories indicated. c) No significant difference between the number of DEGs within iSPNs and dSPNs that are cell-autonomous vs non-cell-autonomous ( $p=0.0975$, two-sided Fisher's exact test). d) There is a significant difference in the number of DEGs within Foxp $1^{D D}$ mice 
that overlap with Foxp $1^{D 2}$ or Foxp $1^{D 1}$ DEGs to unique Foxp1 ${ }^{D D}$ DEGs (interaction DEGs) $(p<0.0001$, two-sided Fisher's exact test). e-f) Enrichment of upregulated or downregulated iSPN-DEGs (e) or dSPN-DEGs (f) across Foxp1 cKO samples in distinct SPN subtypes (top 50 most enriched genes/cluster) using a hypergeometric overlap test (8,000 genes used as background).

\section{Fig. 5. Deletion of Foxp1 in iSPNs alters projection patterns of both dSPNs and iSPNs. a)}

Striatal area quantification of four serial slices from anterior to posterior at $400 \mathrm{um}$ increments within Foxp $1^{C T L}$, Foxp $1^{D 1}$, and Foxp $1^{D 2}$ adult mice. Data are represented as mean \pm SEM, $\mathrm{n}=3-4$ mice/genotype. ${ }^{* * *} \mathrm{p}<0.001$, one-way ANOVA with Tukey's multiple comparisons test. b-d) Representative Tissuecyte 1000 coronal section showing the projections of dSPNs and iSPNs using $D 1^{\text {tdTom }}$ and $D 2^{\text {eGFP }}$ reporter mice, respectively, crossed to $F o x p 1^{C T L}(\mathbf{b}), F_{o x p} 1^{D 1}(\mathbf{c})$, or Foxp $1^{D 2}(\mathbf{d})$.

e) Quantification of the normalized probability maps of iSPN (eGFP) projections within Foxp ${ }^{\text {CTL }}$, Foxp $1^{D 1}$, and Foxp $1^{D 2}$ mice showing reduced GPe projections from iSPNs within Foxp $1^{D 2}$ mice. No significant changes were seen in projection patterns onto the SNc or SNr. Data are represented as mean \pm SEM, $n=3-4$ mice/genotype. ${ }^{* *} \mathrm{p}<0.0001$, two-way ANOVA with Dunnett's multiple comparisons test. f) Quantification of the normalized probability maps of dSPN (tdTomato) projections within Foxp $1^{C T L}$, Foxp $1^{D 1}$, and Foxp $1^{D 2}$ mice showing reduced GPi projections from dSPNs within Foxp $1^{D 2}$ mice. Data are represented as mean \pm SEM, $n=2-4$ mice/genotype. ${ }^{* *} \mathrm{p}<0.01$, two-way ANOVA with Dunnett's multiple comparisons test. g) Schematic of cell-autonomous and non-cellautonomous projection deficits found in the Foxp $1^{D 2}$ animals. h) Overlap of dSPN-DEGs within Foxp $1^{D 1}$ or Foxp $1^{D 2}$ cells. Unique Foxp $1^{D 2}$ dSPN-DEGs that are involved in neuron projection are shown, with ASD-risk genes highlighted in purple. $\mathrm{GPi}=$ globus pallidus internal, $\mathrm{GPe}=$ globus pallidus external, STR= striatum, $\mathrm{SNr}=$ substantia nigra pars reticulata, $\mathrm{SNc}=$ substantia nigra pars compacta. 
Fig. 6. Foxp1 regulates behaviors via distinct striatal circuits. a) Latency to fall was measured on the accelerating rotarod. Foxp $1^{D 2}$ and Foxp $1^{D D}$ mice exhibit significant deficits. Data are represented as mean \pm SEM, $n=11$ Foxp $1^{C T L} ; \mathrm{n}=17$ Foxp ${ }^{D 1} ; \mathrm{n}=18$ Foxp $^{D 2} ; \mathrm{n}=12$ Foxp $1^{D D} .{ }^{*} \mathrm{p}<0.05,{ }^{* *} \mathrm{p}<0.005$, ${ }^{* * *} \mathrm{p}<0.0001$, two-way ANOVA with Sidak's multiple comparisons test. $\left.\mathbf{b}-\mathbf{c}\right)$ Mice were tested within the open field paradigm with velocity (b) and percent time spent in the periphery vs center (c) plotted. Foxp $1^{D 2}$ and $\operatorname{Foxp}^{D D}$ mice had significant increase in activity with no difference in percent time spent in the periphery and center. Data are represented as mean \pm SEM. $n=4$ Foxp $1^{D D} ; n=14$ Foxp $1^{D 1}$; $\mathrm{n}=17$ Foxp $1^{D 2} ; \mathrm{n}=22$ Foxp ${ }^{C T L} .{ }^{* * *} \mathrm{p}<0.0001$, one-way ANOVA with Sidak's multiple comparisons test. d-f) Neonatal isolation vocalizations were measured at P4, P7, and P10. (d) The number of isolation calls were significantly reduced in Foxp $1^{D 1}$ mice. (e) Mean frequency $(\mathrm{kHz})$ of the isolation calls was significantly altered in Foxp $1^{D D}$ mice and at P4 within Foxp $1^{D 1}$ animals. (f) The call slope or "structure" of the call was significantly altered over postnatal development in Foxp $1^{D 1}$ pups and specifically at $\mathrm{P} 10$ within Foxp $1^{D D}$ pups. Data are represented as mean \pm SEM. $\mathrm{n}=11$ Foxp $^{D D} ; \mathrm{n}=47$ Foxp $^{D 1} ; \mathrm{n}=36$ Foxp $1^{D 2} ; \mathrm{n}=71$ Foxp $1^{C T L}$. ${ }^{*} \mathrm{p}<0.05,{ }^{* *} \mathrm{p}<0.005,{ }^{* *} \mathrm{p}<0.0001$, two-way ANOVA with Sidak's multiple comparisons test. g) Representative images of nests. h) Foxp $1^{D 1}$ and $F o x p 1^{D D}$ mice produced nests with significantly lower quality scores compared to Foxp $1^{D 2}$ and $F o x p 1^{D D}$ mice. Data are represented as mean \pm SEM. $\mathrm{n}=5$ Foxp ${ }^{D D} ; \mathrm{n}=4$ Foxp ${ }^{D 1} ; \mathrm{n}=5$ Foxp $1^{D 2} ; \mathrm{n}=7$ Foxp $1^{C T L}$. ${ }^{* *} \mathrm{p}<0.005$, one-way ANOVA with Sidak's multiple comparisons test. i-j) Associative fear memory was assessed using the fear conditioning (FC) paradigm. All Foxp1 cKO mice displays deficits in cued FC (h) shown as the percent of time spent freezing. Only Foxp $1^{D 1}$ and $F o x p 1^{D D}$ mice displayed deficits in contextual FC (i). Data are represented as mean \pm SEM. $n=15$ Foxp $1^{D D} ; n=22$ Foxp $1^{D 1} ; n=11$ Foxp $1^{D 2} ; n=23$ Foxp $1^{C T L}$. ${ }^{*} p<0.05,{ }^{* *} p<0.005,{ }^{* * *} p<0.0001$, two-way ANOVA with Dunnett's multiple comparisons test.

Figure 7. Summary of cellular, structural, functional, and behavioral findings within cell-typespecific Foxp1 conditional knockout mice. Foxp1 ${ }^{D 1}$ mice have an increase in eSPN 
subpopulations, reduced striosomal area, no gross SPN projection deficits, and distinct behavioral deficits relevant to social communication behavior and contextual fear conditioning. Foxp $1^{D 2}$ mice have a marked decrease in iSPN and increase in eSPN subpopulations, reduced striosomal area with few striosomal iSPNs, dSPN and iSPN projection deficits, and distinct behavioral deficits relevant to motor learning and cued fear conditioning.

Supplementary Figure 1. Cell-type annotation of early postnatal striatal scRNA-seq. a) Confocal imaging of the somatosensory cortex of Foxp $1^{C T L}$ or Foxp $1^{D 1}$ adult mice showing reduction of Foxp1 expression within cortical layers V-VI (scale bar is $100 u m, C T X=$ cortex, STR=striatum). b) Heatmap showing the enrichment of genes within each cluster that correlate to a previously annotated dataset (Saunders et al., 2018) using the hypergeometric overlap test. c-d) Expression plots showing the normalized UMI (In) for known marker genes of distinct cell-types: (c) Aqp4 for astrocytes, Olig1 for OPCs, Cx3cr1 for microglia, Flt2 for endothelial, (d) S/c17a7 for glutamatergic cortical neurons, interneuron populations (Chat, Npy), neurogenic and neural differentiation marker (Sox4). (e) Expression plots of markers identifying neurogenic populations: proliferating cells (Mki67), neural progenitors (Asc/1), neural progenitors derived from the lateral ganglionic eminence (D/x2), neurogenic and neural differentiation marker (Sox11). f) Expression plots of genes important for iSPN specification (Sp9), mature SPN marker (Ppp1r1b), and major SPN subtypes (Drd1, Drd2). g) No changes in cell-type composition were observed between the average down-sampled datasets (10 iterations with 9,898 cells within each genotype) compared to the actual dataset.

\section{Supplementary Figure 2. Neuronal subclusters and the intersection of Foxp1 and Foxp2} expressing striatal neurons. a-b) Expression plots with the normalized UMI counts for interneuron marker Nkx2-1 (a) or SPN markers Drd1 (dSPNs) or Drd2 (iSPNs) (b). c) Scatter plots showing the percent expression of enriched transcripts between the largest iSPN (Cluster-2) and dSPN (Cluster-0) 
clusters. d) Upset plot showing the number of cells that overlap in expression of Drd1, Drd2, Foxp1, or Foxp2 transcripts within neurons of control samples. Pie chart inlet shows the percent composition of this overlap (percentages $<1 \%$ not visualized). e-f) Expression plots with the normalized UMI counts for eSPN marker Casz1 (e) and imSPN marker Sox4 (f). g) Coronal striatal image of control animals crossed to both Drd1-tdTomato and Drd2-eGFP reporter mice to label dSPNs or iSPNs,

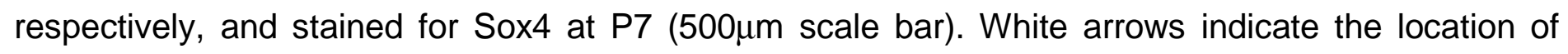

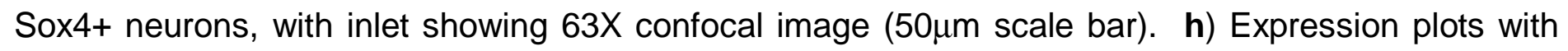
the normalized UMI counts for Foxp2 and Foxp1. i) The same mice from (g) stained for Foxp2. White arrows indicate example cells where Foxp2 does not co-localized with either dSPNs or iSPNs $(50 \mu \mathrm{m}$ scale bar). j) Foxp1 is not expressed within remaining iSPNs within Foxp1 $1^{D 2}$ mice crossed to Drd2eGFP reporter mice at P1, P7, or P56 (adult) timepoints (50 $\mu \mathrm{m}$ scale bar).

\section{Supplementary Figure 3. Confirmation of cell-type-specific targets regulated by Foxp1 and} overlap with ASD-associated genes. a-c) Expression plots of significant DEGs regulated by Foxp1 in both iSPNs and dSPNs (Pde1a), iSPNs (Ppp1r1b), or dSPNs (Calb1). d) Violin plots showing the average normalized UMI (In) of significant DEGs across genotype within all neuronal clusters of Pde1a Ppp1r1b, and Calb1. e) 63X confocal images of coronal, striatal sections stained for Pde1a, Calb1, and Darpp32 in Foxp1 ${ }^{C T L}$ and Foxp1 $1^{D D}$ mice crossed to reporter mice labelling dSPNs with

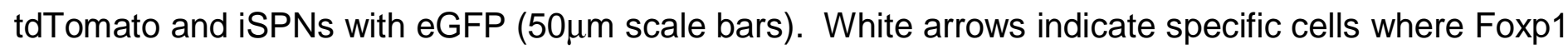
is either 1) upregulating a target (Pde1a) in both dSPNs and iSPNs, 2) upregulating a target (Calb1) in dSPNs only, or 3) downregulating a target (Darpp32) in iSPNs only. f) Enrichment of ASD-risk genes SFARI score 1-4 with upregulated or downregulated iSPN-DEGs (blue) or dSPN-DEGs (red) across Foxp1 cKO samples using a hypergeometric overlap test (8,000 genes used as background).

Supplementary Figure 4. Supplemental behavioral testing of Foxp1 cKO mice. a-b) No change in forelimb (a) or hindlimb (b) grip strength was detected across Foxp1 cKO mice. Data are 
represented as mean \pm SEM. $n=12$ Foxp $^{D D} ; n=17$ Foxp $^{D 1} ; n=16$ Foxp $^{D 2} ; n=11$ Foxp $1^{\text {CTL }}$. Forelimb: $\mathrm{p}=0.8520 \quad\left(\right.$ Foxp $\left.^{D 1}\right), \mathrm{p}=0.6477 \quad\left(\right.$ Foxp $\left.^{12}\right), \mathrm{p}=0.999 \quad\left(\right.$ Foxp $\left.^{D D}\right)$; Hindlimb: $\mathrm{p}=0.7225 \quad\left(\right.$ Foxp $\left.^{D 1}\right)$, $\mathrm{p}=0.6786\left(F_{o x p} 1^{D 2}\right), \mathrm{p}=0.999\left(F o x p 1^{D D}\right)$, one-way ANOVA with Dunnett's multiple comparisons test. c-f) Digigait analysis examining propel stance (c), shared stance (d), propel stride (e), or brake stance (f) across Foxp1 cKO mice. Only Foxp $1^{D 1}$ mice exhibited a significant increase in left forelimb propel stance, propel stride, shared stance, and decrease in left forelimb break stance compared to control animals. Data are represented as mean \pm SEM. $n=7$ Foxp $1^{D D} ; n=9$ Foxp $1^{D 1} ; n=10$ Foxp $1^{D 2}$; $\mathrm{n}=10$ Foxp ${ }^{C T L} \cdot{ }^{*} \mathrm{p}<0.05$, two-way ANOVA with Dunnett's multiple comparisons test. g) Activity levels within a novel-cage environment were unaltered in Foxp1 $c K O$ mice. Data are represented as mean \pm SEM. $\mathrm{n}=7$ Foxp $1^{D D} ; \mathrm{n}=9$ Foxp $1^{D 1} ; \mathrm{n}=10$ Foxp $1^{D 2} ; \mathrm{n}=10$ Foxp $1^{C T L} \cdot \mathrm{p}=0.6834\left(\right.$ Foxp $\left.^{D 1}\right), \mathrm{p}=0.8145$ $\left(F_{o x p 1} 1^{D 2}\right), \mathrm{p}=0.9374$ (Foxp1 $\left.{ }^{D D}\right)$, one-way ANOVA with Dunnett's multiple comparisons test. $\mathbf{h}$ ) Confocal images of striatal sections stained for Mu-Opiod Receptor (MOR) across adult Foxp1 cKO

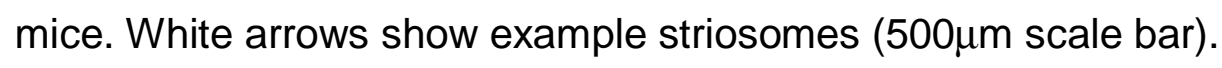

Supplementary Table 1. Differentially expressed genes within clusters. Significantly enriched genes within each cluster identified within the total scRNA-seq dataset (all-cells) and the neuron specific re-clustering dataset. Columns show the gene name, average log fold change, cluster affiliation, percent expressed within that cluster, adjusted p-value.

\section{Supplementary Table 2. Cluster cell-type affiliation and number of cells within each cluster}

across genotype. The total number of clusters, total number of cells within each cluster across genotype, and cluster-cell-type affiliation are shown within respective columns for all-cells and neuron specific datasets. 


\section{Supplementary Table 3. Pairwise differential gene expression analysis between dSPN and}

iSPN subclusters. Differential gene expression analysis was performed between each main subcluster within control dSPNs (Clusters-0, 5, 9) or iSPNs (Clusters-2, 8).

\section{Supplementary Table 4. Pseudobulk differential gene expression analysis between individual}

Foxp1 cKO dSPNs or iSPNs relative to control dSPNs or iSPNs. All significant, differentially expressed genes uncovered in the pseudobulk analysis within iSPNs or dSPNs across Foxp1 cKO samples relative to the same cell-type in control samples. D1cKOs $=F o x p 1^{D 1}$ samples, D2cKOs= Foxp $1^{D 2}$ samples, DDcKOs $=$ Foxp $1^{D D}$ samples.

\section{Supplementary Table 5. Gene ontology analyses of DEGs within dSPNs or iSPNs found in}

Foxp1 cKO samples. GO analysis of Foxp1 regulated DEGs using Toppgene

(https://toppgene.cchmc.org).

\section{Acknowledgments}

Our sincerest thanks to Dr. Helen Lai, Dr. Jane Johnson, Dr. Said Kourrich, Marissa Co, and Dr. Fatma Ayhan for providing critical feedback on the manuscript. G.K. is a Jon Heighten Scholar in Autism Research at UT Southwestern. This work was supported by NIH/NIMH (T32-MH076690) to A.A, the Simons Foundation (SFARI 573689 and 401220), the James S. McDonnell Foundation $21^{\text {st }}$ Century Science Initiative in Understanding Human Cognition - Scholar Award (220020467), the Chan Zuckerberg Initiative, an advised fund of Silicon Valley Community Foundation (HCA-A-170401747), and grants from the NIH (DC014702, DC016340, MH102603) to G. K. We thank Dr. Denise Ramirez, Dr. Julian Meeks, and Apoorva Ajay from the UT Southwestern Whole Brain Microscopy Facility (WBMF) for assistance with volumetric whole brain imaging and automated image analysis. The WBMF is supported by the Texas Institute of Brain Injury and Repair and the UTSW Peter O'Donnell, Jr. Brain Institute. We thank the Neuroscience Microscopy Facility, supported by the UT 
Southwestern Neuroscience Department and the UTSW Peter O'Donnell, Jr. Brain Institute. We would also like to thank Dr. Shari Birnbaum at the UTSW Rodent Behavior Core for help performing the fear conditioning, novel-cage activity, and digigait analyses.

\section{Author contributions}

A.A and G.K designed the study and wrote the paper. A.A performed all single-cell sequencing experiments and library preparations. A.A performed all RNA/protein quantification analyses, immunohistochemistry experiments, and mouse behavior. A.K developed the manual pipeline for scRNA-seq analysis. A.A and A.K analyzed the data. M.H contributed to mouse husbandry and genotyping.

\section{Methods}

\section{Mice}

All experiments were performed according to procedures approved by the UT Southwestern Institutional Animal Care and Use Committee. Foxp $1^{\text {floxfllox }}$ mice ${ }^{72}$ were provided by Dr. Haley Tucker and backcrossed to $\mathrm{C} 57 \mathrm{BL} / 6 \mathrm{~J}$ for at least 10 generations to obtain congenic animals as previously described $^{38,39}$. Drd1a-Cre (262Gsat, 030989-UCD) and Drd2-Cre (ER44Gsat, 032108-UCD) mice were obtained from MMRC. Drd2-eGFP ${ }^{36}$ and Drd1-tdTomato ${ }^{73}$ mice were provided by Dr. Craig Powell. We bred individual Cre or reporter lines to Foxp $1^{\text {floxflox }}$ mice to obtain all Foxp1 cKO mice in one litter that were heterozygous for Cre or reporter transgene. Mice used for single-cell RNAsequencing and behavior experiments were not crossed with Drd1- or Drd2-reporter mice. Reporter mice were crossed with Foxp1 cKO lines for immunohistochemistry experiments and neuronal projection quantification. Mice were maintained on a 12-hr light on/off schedule.

\section{Protein isolation and immunoblotting}


Striatal tissue was dissected, flash frozen, and stored at $-80 \mathrm{C}$ before protein extraction. Protein was extracted from tissue using 1X RIPA Buffer $(750 \mathrm{mM} \mathrm{NaCl}, 250 \mathrm{mM}$ Tris- $\mathrm{HCl} \mathrm{pH} 7.4,0.5 \%$ SDS, $5 \%$ Igepal, 2.5\% Sodium deoxycholate, 5mM EDTA, 5mM NaVO4) with fresh protease inhibitor cocktail $(10 \mathrm{ul} / \mathrm{ml}), 10 \mathrm{ul} / \mathrm{ml}$ of $100 \mathrm{mM} \mathrm{PMSF}$, and $25 \mathrm{ul} / \mathrm{ml}$ of $200 \mathrm{mM}$ sodium orthovanadate. Tissue was homogenized in RIPA buffer using the TissueLyser LT (Qiagen) with a sterile, stainless-steel bead for $1 \mathrm{~min}$ at $50 \mathrm{~Hz}$. Samples were agitated for $1 \mathrm{hr}$ at $4 \mathrm{C}$, spun down at 12,000 rpm for $15 \mathrm{~min}$, and supernatant was transfer to a fresh tube. Protein was quantified using a standard Bradford assay (Bio-Rad) and 20ug of protein per sample were run on 10\% SDS-Page gels. PVDF membranes (BioRad, 162-0177) were incubated in blocking solution (1\% Skim milk in TBS with $0.1 \%$ Tween-20) for $30 \mathrm{~min}$ at room temperature (RT) and probed with primary antibodies overnight at $4 \mathrm{C}$. Membranes were washed with TBS-T (TBS with $0.1 \%$ Tween-20) and incubated with appropriate, species-specific fluorescent secondary antibodies in blocking solution for $1 \mathrm{hr}$ at RT, and washed in TBS-T. Images were collected using the Odyssey infrared imaging system (LI-COR Biosciences).

\section{RNA isolation and quantitative real-time PCR}

RNA from fresh or flash frozen tissue was harvested using miRNAeasy kit guidelines. RNA was converted to cDNA using recommended guidelines from SSIII Superscript Kit (Invitrogen) and qRTPCR was performed using the CFX384 Real-Time System (Bio-Rad).

\section{Immunohistochemistry}

For P7 or P9 mice, rapid decapitation was performed. Brains were extracted and dropped into icecold PBS for $1 \mathrm{~min}$ before transfer into 4\% PFA overnight. Brains were then transferred to $30 \%$ sucrose for 48 hours. 35um coronal slices were made using a SM2000 R sliding microtome (Leica) and free-floating sections were stored in PBS with $0.01 \%$ sodium azide. Slices were washed with TBS and incubated for 30min in 3\% hydrogen peroxide in PBS, washed, then incubated in 30min in $3 \mathrm{M}$ glycine in $0.4 \%$ Triton-X, TBS. Slices were incubated in primary antibodies overnight at $4 \mathrm{C}$, 
washed, and incubated in secondary antibodies for $1 \mathrm{hr}$ at room temperature. Slices were washed then mounted onto slides and allowed to dry overnight. Sections were incubated in DAPI solution (600nM in PBS) on the slide for 5 minutes and washed 3X with PBS. Sections were allowed to dry before mounting coverslips using Prolong Diamond Antifade Mountant.

\section{Imaging and Analysis}

Images were collected using a Zeiss Confocal laser scanning microscope (LSM880) and all image quantification was performed using Fiji image processing package. For iSPN quantification, 20X zstack images of dorsolateral, dorsomedial, and ventral striatum were taken within one hemisphere of four separate striatal sections from anterior to posterior per animal (3 images/section, 4 sections/animal, at least 3 animals/genotype). All images were taken within approximately similar sections across samples. Maximum projection images were quantified within a $1024 \times 1024$ pixel field of view across all images and averaged per section. For striosome quantification, 10X z-stack images were taken from one hemisphere of four separate striatal section from anterior to posterior per animal (4 sections/animals, at least 3 animals/genotype). Individual MOR+ patches were numbered, and area measurements summed for the total striosomal area measurement per section. Total striatal area was also measured per section to calculate the percentage of striosome area to total area per section. Differences between genotypes were assessed using a one-way ANOVA with multiple comparisons.

\section{Antibodies}

The following primary antibodies were used for either immunoblots (IB) or immunohistochemistry (IHC) experiments: chicken anti-GFP (1:1,000, Aves Labs, GFP-1010), rabbit polyclonal anti-MOR (1:350, Millipore, AB5511), rabbit polyclonal anti-PDE1A (1:500, Proteintech, 12442-2-AP), rabbit polyclonal anti-DARPP32 (1:1,000, Millipore, AB1778), goat anti-tdTomato (1:500, LifeSpan Biosciences, LS-C340696), mouse monoclonal anti-FOXP1 (1:500, Abcam, ab32010), rabbit 
polyclonal anti-FOXP1 (IHC:1:1,000, IB: 1:5,000 ${ }^{74}$ ), rabbit polyclonal anti-Calbindin (1:500, Millipore AB1778), goat anti-FOXP2 (N-terminal) (1:500, Santa Cruz 21069), rabbit polyclonal anti- $\beta$-Tubulin (IB: 1:10,000, Abcam, ab243041), and mouse monoclonal anti-SOX4 (1:500, Abcam, ab243041). All IHC following secondary antibodies were used at a 1:1,000 dilutions Alexa Fluor 488 Donkey AntiChicken IgG (Thermo Fisher, 703-545-155), Alexa Fluor 555 Donkey Anti-Goat IgG (Thermo Fisher, A-21432), Alexa Fluor 647 Donkey Anti-Rabbit IgG (Thermo Fisher, 711-605-152), Alexa Fluor 647 Donkey Anti-Mouse IgG (Thermo Fisher, A-31571). For IB, the following secondary antibodies were used at a 1:10,000 dilution: IRDye 800CW Donkey anti-Rabbit IgG (Licor, 925-32213) and IRDye 680RD Donkey anti-Rabbit IgG (Licor, 925-68071).

\section{Tissue processing for single-cell RNA-sequencing (scRNA-seq)}

Mice (P9) were sacrificed by rapid decapitation and brains were quickly removed and placed in ACSF

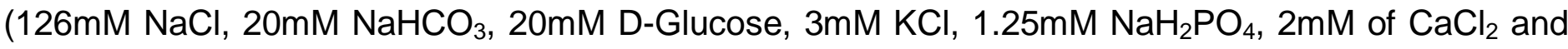
$\mathrm{MgCL}_{2}$ freshly added) bubbled with $95 \% \mathrm{O}_{2}$ and $5 \% \mathrm{CO}_{2}$. Coronal slices at 500 um were made using a VF-200 Compresstome in ACSF and transferred to a recovery chamber at room temperature in ACSF with 50uM AP5, 20uM DNQX, and 100nM TTX $(A C S F+c b)^{75}$. Striatal punches were taken from these slices and incubated in $1 \mathrm{mg} / \mathrm{ml}$ of pronase in $\mathrm{ACSF}+\mathrm{cb}$ for $5 \mathrm{~min}$. Punches were washed with ACSF+ 0.04\% BSA twice and gently dissociated into single-cell suspension using polished Pasteur pipettes with 600um, 300um, and 150um opening diameters, sequentially. Cells were centrifuged and washed twice, filtered through Flowmi Tip 40uM strainers, and resuspended with ACSF+ 0.04\% BSA. Cell viability was quantified using the trypan blue exclusion method and cell concentration was adjusted for targeted sequencing of 10,000 cells/sample using the 10X Genomics Single Cell 3' Reagent Kits v2 protocol to prepare libraries ${ }^{40}$. A total of 16 mice (4 mice/genotype, 2 males and 2 females per genotype) were processed for single-cell sequencing. Libraries were sequenced using the McDermott Sequencing Core at UT Southwestern. 


\section{Pre-processing of Sequencing Data}

Raw sequencing data was acquired from the McDermott Sequencing Core at UT Southwestern in the form of binary base call (BCL) files. BCL files were then de-multiplexed with the 10X Genomics i7 index (used during library preparation) using Illumina's bcl2fastq v2.17.1.14 ${ }^{76}$ and mkfastq command from 10X Genomics CellRanger v2.1.1 tools ${ }^{40}$. Extracted paired-end fastq files (26 bp long R1 - cell barcode and UMI sequence information, 124 bp long R2 - transcript sequence information) were checked for read quality using FASTQC v0.11.5. R1 reads were then used to estimate and identify real cells using whitelist command from UMI-tools v0.5.4 $4^{77}$ program. A whitelist of cell-barcodes (putative real cells) and $\mathrm{R} 2$ fastq files were later used to extract reads corresponding to real cells only (excluding sequence information representing empty beads, doublets, low quality/degrading cells, etc.) using extract command from UMI-tools v0.5. $4^{77}$. This step also appends the cell-barcode and UMI sequence information from $\mathrm{R} 1$ to read names in $\mathrm{R} 2$ fastq file. Extracted $\mathrm{R} 2$ reads were then aligned to reference mouse genome (MM10/GRCm38p6) from UCSC genome browser ${ }^{78}$ and reference mouse annotation (Gencode vM17) using STAR aligner v2.5.2 $\mathrm{b}^{79}$ allowing up to 5 mismatches. Uniquely mapped reads were then assigned to exons using featureCounts program from Subread package $(\mathrm{v} 1.6 .2)^{80}$. Assigned reads sorted and indexed using Samtools v1.6 $6^{81}$ were then used to generate raw expression UMI count tables using count command from UMI-tools v0.5.4 $4^{77,82}$ program. This raw expression matrix contains cells as rows and genes as columns and can be further used for downstream analysis such as normalization, clustering, differentially expressed genes, etc.

\section{Clustering Analysis}

Raw single-cell RNA-seq UMI count data was used for clustering analysis using Seurat R analysis pipeline $^{83}$. First, cells with more than 50,000 molecules (nUMI per cell) and cells with more than $10 \%$ mitochondrial content were filtered out to discard potential doublets and degrading cells. Also, genes from mitochondrial chromosome and chromosomes $X$ and $Y$ were removed as samples were from mixed genders. This dataset is referred to as primary filtered dataset. Post filtering, the raw UMI 
counts from primary filtered dataset were used for log-normalization and scaled using a factor of 10,000 and regressed to covariates such as number of UMI per cells and percent mitochondrial content per cell as described in Seurat analysis pipeline ${ }^{83}$. To further identify the top variable genes, the data were used to calculate principal components (PCs). Using Jackstraw analysis, statistically significant PCs were used to identify clusters within the data using original Louvain algorithm as described in Seurat analysis pipeline followed by visualizing the clusters with uniform manifold approximation and projection (UMAP) in two dimensions ${ }^{84}$. Genes enriched in each cluster compared to the remainder of the cells (adj. $p$-value $<=0.05$ and log fold change $>=0.3$ ) were identified as described in Seurat analysis pipeline. Genes corresponding to each cluster were used to identify the cell-type by correlating to genes expressed in previously published adult mouse striatal single cell data $^{13}$. Cell-types were assigned to clusters based on (i) statistically significant enrichment of gene sets using the hypergeometric test (with a background of 7,500 genes, the number of expressed genes within our dataset) and (ii) expression weighted cell-type enrichment (EWCE) analysis ${ }^{41}$ (https://github.com/NathanSkene/EWCE). Clusters that overlapped significantly with multiple celltypes were called for the most significant overlap (smallest Adj. P-value) and analyzed for expression of top marker genes of known cell-types. Cells from clusters that fell into neuronal categories (referred to as secondary neuronal dataset) were used to re-cluster the cells to define specific spiny projection neuronal sub-types using a similar approach as described above. Note that two small clusters (Clusters-21, 22) that corresponded to excitatory cortical neurons and a cluster with less than 30 cells total (Cluster-24) were excluded from the secondary neuronal dataset UMAP plots to focus on striatal cell-types.

\section{Differential Gene Expression (DEG) Analyses}

\section{Pairwise DEG analysis SPNs}

For the spiny projection neuronal sub-type clusters identified using secondary neuronal dataset, pairwise differential gene expression analysis tests were performed within each cluster-pair using a 
Poisson likelihood ratio test from the Seurat $R$ analysis pipeline ${ }^{83}$ to identify genes enriched (adj. $p$ value $<=0.05, \log _{2} \mathrm{FC}>|0.25|$ ) in SPN sub-types.

\section{Pseudobulk DEG analysis}

Within the secondary neuronal dataset, neurons identified as either dSPNs (Drd1+) or iSPNs (Drd2+) were combined into pools of cells segregated by genotypes. Differential expression within pools of dSPN or iSPNs of Foxp1 cKO samples were then compared to control samples using Poisson likelihood ratio test from the Seurat $R$ analysis pipeline accounting for averaged expression differences in either dSPNs or iSPNs across genotypes irrespective of the identified clusters. Significant expression changes (adj. p-value $<=0.05, \log _{2} \mathrm{FC}>|0.3|$ ) reflected the differences in expression of genes in one specific cell population (dSPNs or iSPNs) across genotypes instead of detected clusters.

\section{Down-sampled Dataset Analysis}

Cells from the primary filtered dataset were used to randomly select the cells from each genotype matching the number of cells present in each genotype with the lowest representation of the cells $\left(\right.$ Foxp $^{C T L}=14466$ cells, Foxp $1^{D 1}=16,961$ cells, Foxp $1^{D 2}=9,898$ cells, Foxp $1^{D D}=21,453$ cells, using random sampling, the same number of cells from Foxp $1^{C T L}$, Foxp $1^{D 1}$ and $F o x p 1^{D D}$ were matched to Foxp $1^{D 2}$ ). This is referred to as the primary down-sampled dataset. This dataset was further used to separate the cells into clusters and identify cell-types as described in the clustering analysis section above. Clusters corresponding to SPNs from the primary down-sampled dataset (referred to as the secondary down-sampled neuronal dataset) were re-clustered to identify SPN subtypes in a similar manner as described in the clustering section above.

\section{Availability of Data and Code}

The sequencing data reported in this paper can be access at NCBI GEO with accession number GSE125290. Code that was used to perform data pre-processing, clustering and differential gene 
expression analysis is available at GitHub repository (https://github.com/konopkalab/early-postnatalstriatal-single-cell-rna-seq).

\section{TissueCyte Imaging and Quantification}

\section{STPT and image acquisition}

Serial two-photon tomography (STPT) ${ }^{49}$, in which automated block face imaging of the brain is repetitively alternated with vibratome sectioning, was conducted on the TissueCyte 1000 platform using the manufacturer's custom software for operation (Orchestrator). Mouse brains were perfusionfixed in $4 \%$ paraformaldehyde and embedded in low-melting point oxidized agarose $(4.5 \% \mathrm{w} / \mathrm{v}$; Sigma \#A0169). Vibratome sections were prepared at $75 \mu \mathrm{m}$ thickness using a frequency of $70 \mathrm{~Hz}$ and a speed of $0.5 \mathrm{~mm} / \mathrm{sec}$. $185-190$ total sections were collected of each brain. A 9 by 13 mosaic of tile images was collected at each level using lateral resolution of $0.875 \mu \mathrm{m} /$ pixel. Optical sectioning was used to collect three z-planes within each $75 \mu \mathrm{m}$ physical section to obtain $25 \mu \mathrm{m}$ axial resolution. The two-photon excitation laser (Spectra Physics MaiTai DeepSee) was tuned to $920 \mathrm{~nm}$ to excite both eGFP and tdTomato. The emission fluorescence from the red, green and blue channels was independently collected using photomultiplier tube detectors. The tile images were saved to network attached servers and automatically processed to perform flat field correction and then stitched into single-channel 2D coronal sections in 16-bit .tif format using the manufacturer's custom software (AutoStitcher).

\section{Sample preparation and details}

Mice ( 8 weeks) were perfused with PBS followed by 4\% PFA. Brains were removed and post-fixed overnight in $4 \%$ PFA at $4 \mathrm{C}$. Samples were transferred to PBS $+0.1 \%$ sodium azide and stored at $4 \mathrm{C}$ until imaging. A total of 19 whole mouse brain images were collected in three cohorts for machine learning analysis according to their patterns of fluorophore expression. The first cohort consisted of 8 samples expressing tdTomato (detected predominantly in the red channel), the second cohort had 8 
samples that expressed eGFP (detected predominantly in the green channel) and the third cohort consisted of 3 dual-labeled (eGFP + tdTomato) samples.

\section{TissueCyte image processing and registration}

STPT image processing was performed via BioHPC, an advanced computing cluster at UT Southwestern. All channels of the coronal sections were downsampled to $10 \mu \mathrm{m}$ lateral resolution, intensity adjusted to fill the 16-bit range, and combined to form 3D image stacks using custom MATLAB software. The image stacks were then processed through a 3D median filter to remove high-contrast noise. The 3D image stacks were registered to Allen Institute for Brain Science Common Coordinate Framework (version 3, CCFv3) at $10 \mu \mathrm{m} \times 10 \mu \mathrm{m} \times 100 \mu \mathrm{m}$ resolution using NiftyReg software ${ }^{85}$. Briefly, registration involved three steps: (i) Affine transformation (reg-aladin) for global registration (ii) Cubic B-spline transformation (reg-f3d) to achieve local transformation and (iii) Resampling the transformed brains to Atlas coordinates (reg-resample). Registration transformations were established based on the red channel, then applied equally to all other data channels, including the probability maps (described below).

\section{Interactive Image training for classifying signals of interest}

The three raw channels of the $2 \mathrm{D}$ stitched coronal sections were downsampled to $1.5 \mu \mathrm{m}$ lateral resolution. A maximum intensity projection of the three optical sections was produced for each physical section across all 3 color channels, creating an RGB image stack with the same number of 2D frames as physical sections (e.g. 185 or 190). llastik (Interactive learning and segmentation toolkit ${ }^{50}$ software was deployed on BioHPC and used to train a pixel-wise random forest classifier to identify features of interest (e.g. fluorescent neuronal cell bodies and axonal projections). Three or four representative sections were chosen from the 185-190 image stack for model training. A supervised random forest model was trained by users to classify fluorescent features of interest (e.g. eGFP and/or tdTomato), and to distinguish them from other image features (e.g., bright microbubbles, empty space, autofluorescence) using the interactive features in llastik. An independent random forest model was trained for each of the image batches described above. The random forest 
classifiers were used to detect features of interest in all image sections, creating a "probability map" for each voxel in each 3D whole brain image. In these probability map images, the value of each voxel in each virtual channel (corresponding to each image feature, e.g. eGFP) represents the probability that the voxel includes information for the desired feature. These exported probability maps were registered to the CCFv3.0 using the transformation parameters using NiftyReg (regaladin).

\section{Quantification and visualization}

The features of interest in the registered probability maps were quantified by automatically segmenting brain regions of interest based upon CCFv3.0 volumetric annotations. Custom MATLAB software aggregated brain regions of interest (e.i., nucleus accumbens, caudate putamen, globus pallidus external and internal, substantia nigra pars compacta and pars reticulata), calculated the cumulative probabilities of all voxels in each region, and normalized these values by the volume of each structure. This exported data matrix thus included normalized probability intensity values for each machine learning feature, each brain region of interest, and each brain. For visualization, the combined probability map stacks were rendered in 3D using the ClearVolume plugin for Fiji/lmage $J^{86}$.

\section{Behavior tests}

\section{Open Field}

Mice age 8-12 weeks were allowed to acclimate to the testing room for $1 \mathrm{hr}$ before being placed in a $55 \mathrm{~cm} \times 55 \mathrm{~cm} \times 36 \mathrm{~cm}$ matrix (Phenome Technologies) and recorded for $30 \mathrm{~min}$. Total distance and velocity measurements were analyzed using Actimetrics LimeLight software.

\section{Novel-cage activity}

As previously described ${ }^{38}$, mice were moved into individual cages $(18 \times 28 \mathrm{~cm})$ with minimal bedding. Cage was placed into a dark Plexiglas box and the movements were measured using a Photobeam Activity System-Home Cage software for two hours. The number of beam breaks was recorded every 5 min and averaged over two hours for statistical analyses. 


\section{Rotarod}

Following previously published methods ${ }^{35}$, mice (8-12 weeks) were acclimated to the testing room for $30 \mathrm{~min}$ before placed in one lane of a 5-lane accelerating rotarod (Series 8 ITCC Life Science rotarod). The textured drum within the individual lanes was programed to accelerate from acceleration from 4-40 rpm within a maximum time frame of $300 \mathrm{sec}$. Each mouse was positioned facing away from the experimenter. Latency to fall was recorded once the trial was initiated. Manual activation of the sensors occurred when an animal made a full rotation holding onto the drum. Animals received four trials per day (20min intervals) with lanes cleaned between animals with NPD over the course of three consecutive days.

\section{Grip strength test}

Grip strength was tested following previously published methods ${ }^{35}$. Briefly, following rotorad experiments, the forelimb and hindlimb grip strength mice were measured using Chatillon Force Measurement equipment. The forelimbs, followed by the hindlimbs, for each animal were tested first by placing forelimb paws on a mesh wire meter and pulling them away from the wire at constant force. Five consecutive measurements were recorded for both hindlimbs and forelimbs and averaged for a final grip strength measurement.

\section{Nestlet behavior}

Nesting behavior was analyzed using a previously published approach ${ }^{38,52}$. Mice (8-12 weeks) were isolated into clean cages overnight with $3 \mathrm{~g}$ of intact nestlet. After 16-18 hrs, the amount of unused nestlet was measured and images of the nests were taken to assess the quality and given a score.

\section{Neonatal ultrasonic vocalization measurements}

USVs were recorded as described previously ${ }^{35,38}$. Briefly, pups were isolated from dams at P4, P7, and P10 and placed into a soundproof container. USVs were recorded for 3min with an UltraSoundGate condenser microphone using Avisoft Bioacoustic software. Analysis of sound spectrograms was automatically performed using MATLAB $\operatorname{codes}^{87}$.

\section{Digigait}


Mice (8-12 weeks) were placed onto the transparent treadmill using the DigiGait Imaging System (Mouse Specifics, Inc) at $10 \mathrm{~cm} / \mathrm{sec}$. The speed was quickly increased to $20 \mathrm{~cm} / \mathrm{sec}$ with a highspeed video camera mounted under the clear treadmill to capture images of all four paws at the 20 $\mathrm{cm} / \mathrm{sec}$ speed. A section of video with at least 6-10 steps is analyzed and the paw placement is automatically detected and quantified by the software system. Right and left forelimb and hindlimb paw measurements were analyzed separately.

\section{Fear Conditioning}

Fear conditioning was measured using boxes with metal grid floors connected to a scrambled shock generator (Med Associates Inc., St. Albans). Mice were trained by placing them individually in the chamber for $2 \mathrm{~min}$ before they received 3 tone-shock pairings (30sec white noise, $80 \mathrm{~dB}$ tone, coterminated with a $2 \mathrm{sec}, 0.5 \mathrm{~mA}$ footshock, $1 \mathrm{~min}$ intertrial interval). Twenty-four hours later, contextual memory was measured by placing the mice into the same chamber and measuring freezing behavior using the Med Associates software. Forty-eight hours post training, memory of the white noise cue was measured by placing mice in new environment, with altered floors, walls, different lighting, and a vanilla smell. Freezing was measured for $3 \mathrm{~min}$ and then noise cue was turned on for an additional 3 min and freezing was measured.

\section{Statistics and reproducibility}

Statistical methods and code used for scRNA-seq and analysis are provided in the above methods sections. All statistical test used (and p-values obtained) for SPN projection analysis, behavior, and immunohistochemistry are described in figure legends. No statistical methods were used to estimate sample size, but behavior cohorts were based on previously published papers ${ }^{35,38,39}$. Sample size for each experiment is indicated in figure legends.

\section{References}


bioRxiv preprint doi: https://doi.org/10.1101/611780; this version posted April 18 2019. The copyright holder for this preprint (which was not

certified by peer review) is the author/funder, who has granted bioRxiv a license to display the preprint in perpetuity. It is made available under aCC-BY-ND 4.0 International license.

1. Gerfen, C. R. \& Surmeier, D. J. Modulation of Striatal Projection Systems by Dopamine. Annu. Rev. Neurosci. 34, 441-466 (2011).

2. Cui, G. et al. Concurrent activation of striatal direct and indirect pathways during action initiation. Nature 494, 238-242 (2013).

3. Tecuapetla, F., Jin, X., Lima, S. Q. \& Costa, R. M. Complementary Contributions of Striatal Projection Pathways to Action Initiation and Execution. Cell 166, 703-715 (2016).

4. Crittenden, J. R. \& Graybiel, A. M. Basal Ganglia disorders associated with imbalances in the striatal striosome and matrix compartments. Front Neuroanat 5, 59 (2011).

5. Fuccillo, M. V. Striatal Circuits as a Common Node for Autism Pathophysiology. Front Neurosci 10, 27 (2016).

6. Crittenden, J. R. et al. Striosome-dendron bouquets highlight a unique striatonigral circuit targeting dopamine-containing neurons. Proc. Natl. Acad. Sci. U.S.A. 113, 11318-11323 (2016).

7. Friedman, A. et al. A Corticostriatal Path Targeting Striosomes Controls Decision-Making under Conflict. Cell 161, 1320-1333 (2015).

8. Smith, J. B. et al. Genetic-Based Dissection Unveils the Inputs and Outputs of Striatal Patch and Matrix Compartments. Neuron 91, 1069-1084 (2016).

9. Kelly, S. M. et al. Radial Glial Lineage Progression and Differential Intermediate Progenitor Amplification Underlie Striatal Compartments and Circuit Organization. Neuron 99, 345-361.e4 (2018).

10. Lobo, M. K., Karsten, S. L., Gray, M., Geschwind, D. H. \& Yang, X. W. FACS-array profiling of striatal projection neuron subtypes in juvenile and adult mouse brains. Nat Neurosci 9, 443-452 (2006).

11. Heiman, M. et al. A Translational Profiling Approach for the Molecular Characterization of CNS Cell Types. Cell 135, 738-748 (2008).

12. Gokce, O. et al. Cellular Taxonomy of the Mouse Striatum as Revealed by Single-Cell RNASeq. CellReports 16, 1126-1137 (2016).

13. Saunders, A. et al. Molecular Diversity and Specializations among the Cells of the Adult Mouse Brain. Cell 174, 1015-1030.e16 (2018).

14. Anderson, S. A. et al. Mutations of the homeobox genes Dlx-1 and DIx-2 disrupt the striatal subventricular zone and differentiation of late born striatal neurons. Neuron 19, 27-37 (1997).

15. Garel, S., Marín, F., Grosschedl, R. \& Charnay, P. Ebf1 controls early cell differentiation in the embryonic striatum. Development 126, 5285-5294 (1999).

16. Corbin, J. G., Gaiano, N., Machold, R. P., Langston, A. \& Fishell, G. The Gsh2 homeodomain gene controls multiple aspects of telencephalic development. Development 127, 5007-5020 (2000).

17. Arlotta, P., Molyneaux, B. J., Jabaudon, D., Yoshida, Y. \& Macklis, J. D. Ctip2 Controls the Differentiation of Medium Spiny Neurons and the Establishment of the Cellular Architecture of the Striatum. Journal of Neuroscience 28, 622-632 (2008).

18. Kim, E. J., Battiste, J., Nakagawa, Y. \& Johnson, J. E. Ascl1 (Mash1) lineage cells contribute to discrete cell populations in CNS architecture. Molecular and Cellular Neuroscience 38, 595606 (2008).

19. Lobo, M. K., Yeh, C. \& Yang, X. W. Pivotal role of early B-cell factor 1 in development of striatonigral medium spiny neurons in the matrix compartment. J. Neurosci. Res. 86, 21342146 (2008).

20. Long, J. E. et al. Dlx1\&2 and Mash1 transcription factors control striatal patterning and differentiation through parallel and overlapping pathways. J. Comp. Neurol. 512, 556-572 (2009).

21. Ehrman, L. A. et al. The LIM homeobox gene Isl1 is required for the correct development of the striatonigral pathway in the mouse. Proc. Natl. Acad. Sci. U.S.A. 110, E4026-35 (2013). 
bioRxiv preprint doi: https://doi org/10.1101/611780; this version posted April 18 2019. The copyright holder for this preprint (which was not

certified by peer review) is the author/funder, who has granted bioRxiv a license to display the preprint in perpetuity. It is made available under aCC-BY-ND 4.0 International license.

22. Maze, I. et al. G9a influences neuronal subtype specification in striatum. Nat Neurosci 17, 533539 (2014).

23. Lu, K.-M., Evans, S. M., Hirano, S. \& Liu, F.-C. Dual role for Islet-1 in promoting striatonigral and repressing striatopallidal genetic programs to specify striatonigral cell identity. Proc. Natl. Acad. Sci. U.S.A. 111, E168-77 (2014).

24. Martín-lbáñez, R. et al. Helios expression coordinates the development of a subset of striatopallidal medium spiny neurons. Development 144, 1566-1577 (2017).

25. Waclaw, R. R. et al. Foxo1 is a downstream effector of Isl1 in direct pathway striatal projection neuron development within the embryonic mouse telencephalon. Mol. Cell. Neurosci. 80, 44-51 (2017).

26. Xu, Z. et al. SP8 and SP9 coordinately promote D2-type medium spiny neuron production by activating Six3 expression. Development 145, dev165456 (2018).

27. Kozorovitskiy, Y., Saunders, A., Johnson, C. A., Lowell, B. B. \& Sabatini, B. L. Recurrent network activity drives striatal synaptogenesis. Nature 485, 646-650 (2012).

28. Peixoto, R. T., Wang, W., Croney, D. M., Kozorovitskiy, Y. \& Sabatini, B. L. Early hyperactivity and precocious maturation of corticostriatal circuits in Shank3B(-/-) mice. Nat Neurosci 19, 716-724 (2016).

29. Fong, W. L., Kuo, H.-Y., Wu, H.-L., Chen, S.-Y. \& Liu, F.-C. Differential and Overlapping Pattern of Foxp1 and Foxp2 Expression in the Striatum of Adult Mouse Brain. Neuroscience 388, 214-223 (2018).

30. Meerschaut, I. et al. FOXP1-related intellectual disability syndrome: a recognisable entity. J. Med. Genet. 54, 613-623 (2017).

31. Siper, P. M. et al. Prospective investigation of FOXP1 syndrome. Molecular Autism 8, 57 (2017).

32. Ferland, R. J., Cherry, T. J., Preware, P. O., Morrisey, E. E. \& Walsh, C. A. Characterization of Foxp2 and Foxp1 mRNA and protein in the developing and mature brain. J. Comp. Neurol. 460, 266-279 (2003).

33. Precious, S. V. et al. FoxP1 marks medium spiny neurons from precursors to maturity and is required for their differentiation. Exp. Neurol. 282, 9-18 (2016).

34. Bacon, C. et al. Brain-specific Foxp1 deletion impairs neuronal development and causes autistic-like behaviour. Molecular Psychiatry 20, 632-639 (2015).

35. Araujo, D. J. et al. FoxP1 orchestration of ASD-relevant signaling pathways in the striatum. Genes \& Development 29, 2081-2096 (2015).

36. Gong, S. et al. Targeting Cre recombinase to specific neuron populations with bacterial artificial chromosome constructs. J. Neurosci. 27, 9817-9823 (2007).

37. Feng, X. et al. Foxp1 is an essential transcriptional regulator for the generation of quiescent naive T cells during thymocyte development. Blood 115, 510-518 (2010).

38. Araujo, D. J. et al. Foxp1 in Forebrain Pyramidal Neurons Controls Gene Expression Required for Spatial Learning and Synaptic Plasticity. J. Neurosci. 37, 10917-10931 (2017).

39. Usui, N. et al. Foxp1 regulation of neonatal vocalizations via cortical development. Genes \& Development 31, 2039-2055 (2017).

40. Zheng, G. X. Y. et al. Massively parallel digital transcriptional profiling of single cells. Nature Communications 8, 14049 (2017).

41. Skene, N. G. \& Grant, S. G. N. Identification of Vulnerable Cell Types in Major Brain Disorders Using Single Cell Transcriptomes and Expression Weighted Cell Type Enrichment. Front Neurosci 10, 16 (2016).

42. Bergsland, M., Werme, M., Malewicz, M., Perlmann, T. \& Muhr, J. The establishment of neuronal properties is controlled by Sox4 and Sox11. Genes \& Development 20, 3475-3486 (2006). 
bioRxiv preprint doi: https://doi.org/10.1101/611780; this version posted April 18 2019. The copyright holder for this preprint (which was not

certified by peer review) is the author/funder, who has granted bioRxiv a license to display the preprint in perpetuity. It is made available under aCC-BY-ND 4.0 International license.

43. Chen, C., Lee, G. A., Pourmorady, A., Sock, E. \& Donoghue, M. J. Orchestration of Neuronal Differentiation and Progenitor Pool Expansion in the Developing Cortex by SoxC Genes. J. Neurosci. 35, 10629-10642 (2015).

44. Shu, W., Yang, H., Zhang, L., Lu, M. M. \& Morrisey, E. E. Characterization of a new subfamily of winged-helix/forkhead (Fox) genes that are expressed in the lung and act as transcriptional repressors. Journal of Biological Chemistry 276, 27488-27497 (2001).

45. Vernes, S. C. et al. Foxp2 regulates gene networks implicated in neurite outgrowth in the developing brain. PLoS Genet 7, e1002145 (2011).

46. Thibault, D., Loustalot, F., Fortin, G. M., Bourque, M.-J. \& Trudeau, L.-É. Evaluation of D1 and D2 dopamine receptor segregation in the developing striatum using BAC transgenic mice. PLOS ONE 8, e67219 (2013).

47. Vernes, S. C. et al. A functional genetic link between distinct developmental language disorders. N. Engl. J. Med. 359, 2337-2345 (2008).

48. O'Roak, B. J. et al. Exome sequencing in sporadic autism spectrum disorders identifies severe de novo mutations. Nat Genet 43, 585-589 (2011).

49. Ragan, T. et al. Serial two-photon tomography for automated ex vivo mouse brain imaging. Nat. Methods 9, 255-258 (2012).

50. Sommer, C., Straehle, C., Koethe, U. \& Hamprecht, F. A. Ilastik: Interactive learning and segmentation toolkit. in 230-233 (2011).

51. Silverman, J. L., Yang, M., Lord, C. \& Crawley, J. N. Behavioural phenotyping assays for mouse models of autism. Nature Publishing Group 11, 490-502 (2010).

52. Deacon, R. M. J. Assessing nest building in mice. Nat Protoc 1, 1117-1119 (2006).

53. Ikegami, M., Uemura, T., Kishioka, A., Sakimura, K. \& Mishina, M. Striatal dopamine D1 receptor is essential for contextual fear conditioning. Sci. Rep. 4, 1-10 (2014).

54. Tepper, J. M., Sharpe, N. A., Koós, T. Z. \& Trent, F. Postnatal development of the rat neostriatum: electrophysiological, light- and electron-microscopic studies. Dev. Neurosci. 20, 125-145 (1998).

55. Tunstall, M. J., Oorschot, D. E., Kean, A. \& Wickens, J. R. Inhibitory interactions between spiny projection neurons in the rat striatum. J. Neurophysiol. 88, 1263-1269 (2002).

56. Taverna, S., Ilijic, E. \& Surmeier, D. J. Recurrent collateral connections of striatal medium spiny neurons are disrupted in models of Parkinson's disease. J. Neurosci. 28, 5504-5512 (2008).

57. Tecuapetla, F., Koós, T., Tepper, J. M., Kabbani, N. \& Yeckel, M. F. Differential dopaminergic modulation of neostriatal synaptic connections of striatopallidal axon collaterals. J. Neurosci. 29, 8977-8990 (2009).

58. Tinterri, A. et al. Active intermixing of indirect and direct neurons builds the striatal mosaic. Nature Communications 9, 4725 (2018).

59. Iossifov, I. et al. The contribution of de novo coding mutations to autism spectrum disorder. Nature 515, 216-221 (2016).

60. Stessman, H. A. F. et al. Targeted sequencing identifies 91 neurodevelopmental-disorder risk genes with autism and developmental-disability biases. Nat Genet 49, 515-526 (2017).

61. Durieux, P. F. et al. D2R striatopallidal neurons inhibit both locomotor and drug reward processes. Nat Neurosci 12, 393-395 (2009).

62. Bateup, H. S. et al. Distinct subclasses of medium spiny neurons differentially regulate striatal motor behaviors. Proc. Natl. Acad. Sci. U.S.A. 107, 14845-14850 (2010).

63. Bello, E. P. et al. Inducible ablation of dopamine D2 receptors in adult mice impairs locomotion, motor skill learning and leads to severe parkinsonism. Molecular Psychiatry 22, 595-604 (2016).

64. Boulanger-Bertolus, J., Rincón-Cortés, M., Sullivan, R. M. \& Mouly, A.-M. Understanding pup affective state through ethologically significant ultrasonic vocalization frequency. Sci. Rep. 7, 13483 (2017). 
bioRxiv preprint doi: https://doi org/10.1101/611780; this version posted April 18, 2019. The copyright holder for this preprint (which was not

certified by peer review) is the author/funder, who has granted bioRxiv a license to display the preprint in perpetuity. It is made available under aCC-BY-ND 4.0 International license.

65. Li, S., Weidenfeld, J. \& Morrisey, E. E. Transcriptional and DNA binding activity of the

Foxp1/2/4 family is modulated by heterotypic and homotypic protein interactions. Molecular and Cellular Biology 24, 809-822 (2004).

66. Konopka, G. \& Roberts, T. F. Insights into the Neural and Genetic Basis of Vocal Communication. Cell 164, 1269-1276 (2016).

67. Chang, J., Gilman, S. R., Chiang, A. H., Sanders, S. J. \& Vitkup, D. Genotype to phenotype relationships in autism spectrum disorders. Nat Neurosci 18, 191-198 (2014).

68. Turner, T. N. et al. Genomic Patterns of De Novo Mutation in Simplex Autism. Cell 171, 710722.e12 (2017).

69. Xu, X., Wells, A. B., O'Brien, D. R., Nehorai, A. \& Dougherty, J. D. Cell Type-Specific Expression Analysis to Identify Putative Cellular Mechanisms for Neurogenetic Disorders. Journal of Neuroscience 34, 1420-1431 (2014).

70. Takata, A. et al. Integrative Analyses of De Novo Mutations Provide Deeper Biological Insights into Autism Spectrum Disorder. CellReports 22, 734-747 (2018).

71. Coe, B. P. et al. Neurodevelopmental disease genes implicated by de novo mutation and copy number variation morbidity. Nat Genet 51, 106-116 (2019).

72. Zhang, Y. et al. Foxp1 coordinates cardiomyocyte proliferation through both cell-autonomous and nonautonomous mechanisms. Genes \& Development 24, 1746-1757 (2010).

73. Ade, K. K., Wan, Y., Chen, M., Gloss, B. \& Calakos, N. An Improved BAC Transgenic

Fluorescent Reporter Line for Sensitive and Specific Identification of Striatonigral Medium Spiny Neurons. Front Syst Neurosci 5, 32 (2011).

74. Spiteri, E. et al. Identification of the transcriptional targets of FOXP2, a gene linked to speech and language, in developing human brain. Am. J. Hum. Genet. 81, 1144-1157 (2007).

75. Tasic, B. et al. Adult mouse cortical cell taxonomy revealed by single cell transcriptomics. Nat Neurosci 19, 335-346 (2016).

76. Andrews, S. FastQC: a quality control tool for high throughput sequence data. (2010).

77. Smith, T. S., Heger, A. \& Sudbery, I. UMI-tools: Modelling sequencing errors in Unique Molecular Identifiers to improve quantification accuracy. Genome Res. 27, gr.209601.116-499 (2017).

78. Kent, W. J. et al. The human genome browser at UCSC. Genome Res. 12, 996-1006 (2002).

79. Dobin, A. et al. STAR: ultrafast universal RNA-seq aligner. Bioinformatics 29, 15-21 (2013).

80. Liao, Y., Smyth, G. K. \& Shi, W. featureCounts: an efficient general purpose program for assigning sequence reads to genomic features. Bioinformatics 30, 923-930 (2014).

81. Li, H. et al. The Sequence Alignment/Map format and SAMtools. Bioinformatics 25, 2078-2079 (2009).

82. Smith, T. S., Heger, A. \& Sudbery, I. UMI-tools: Modelling sequencing errors in Unique Molecular Identifiers to improve quantification accuracy. Genome Res. 27, gr.209601.116-499 (2017).

83. Butler, A., Hoffman, P., Smibert, P., Papalexi, E. \& Satija, R. Integrating single-cell transcriptomic data across different conditions, technologies, and species. Nat. Biotechnol. 36, 411-420 (2018).

84. Becht, E. et al. Dimensionality reduction for visualizing single-cell data using UMAP. Nat. Biotechnol. 37, 38-44 (2018).

85. Modat, M. et al. Global image registration using a symmetric block-matching approach. $J$ Med Imaging (Bellingham) 1, 024003 (2014).

86. Royer, L. A. et al. ClearVolume: open-source live 3D visualization for light-sheet microscopy. Nat. Methods 12, 480-481 (2015).

87. Rieger, M. A. \& Dougherty, J. D. Analysis of within Subjects Variability in Mouse Ultrasonic Vocalization: Pups Exhibit Inconsistent, State-Like Patterns of Call Production. Front Behav Neurosci 10, 182 (2016). 
bioRxiv preprint doi: https://doi.org/10.1101/611780; this version posted April 18, 2019. The copyright holder for this preprint (which was not Fig. 1 certified by peer review) is the author/funder, who has granted bioRxiv a license to display the preprint in perpetuity. It is made available under

a

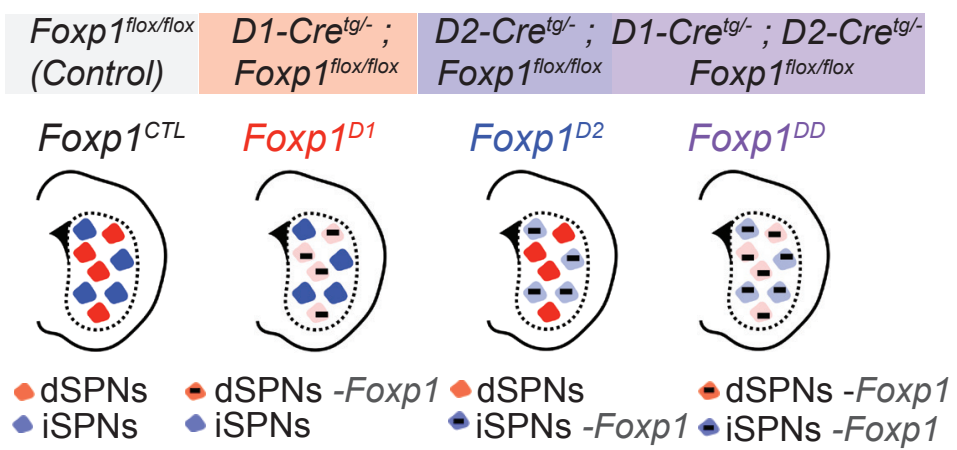

$\begin{aligned} & \text { P9 striatal single-cell } \\ & \text { dissocation }\end{aligned} \mid \mathrm{N}=4 /$ genotype

scRNA-seq prep (10X Genomics) 62,778 cells

e

Median: 3,837 Mean: 5,587

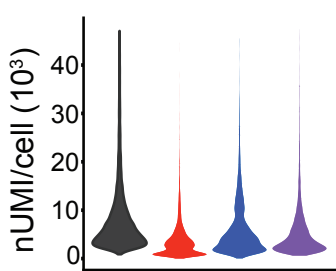

$\square$ Foxp1 $1^{\text {CTL }}$ Foxp1 $1^{D 1}$ Foxp1 $1^{D 2}$ Goxp1DD

f

SPNs

$$
6 \quad 28
$$

Ctx. excitatory

neurons

Inter-
neurons 36
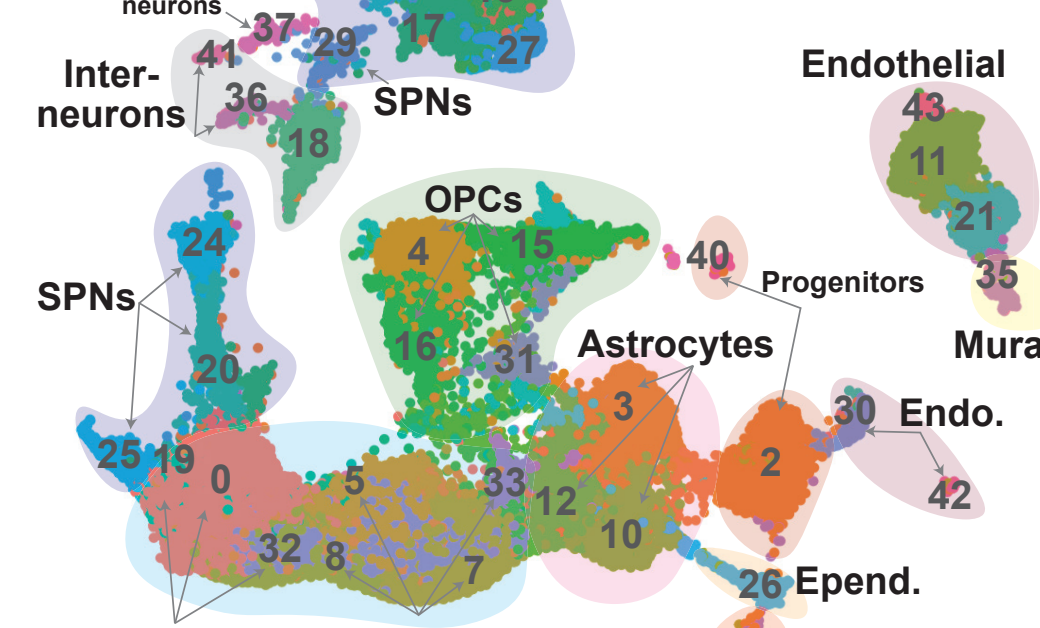

Neurogenic progenitors

Microglia

\section{Endothelial}

Median: 1,532 Mean: 1,794
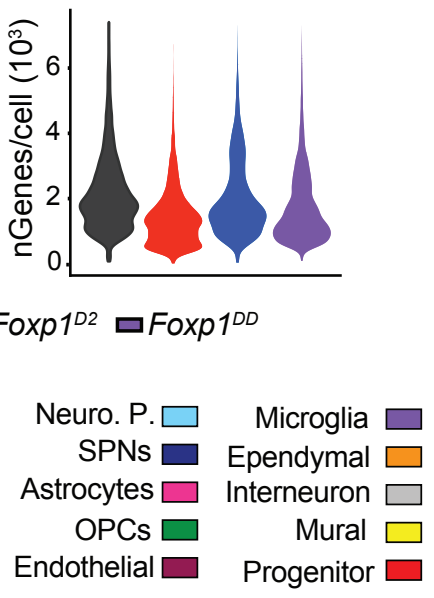

C

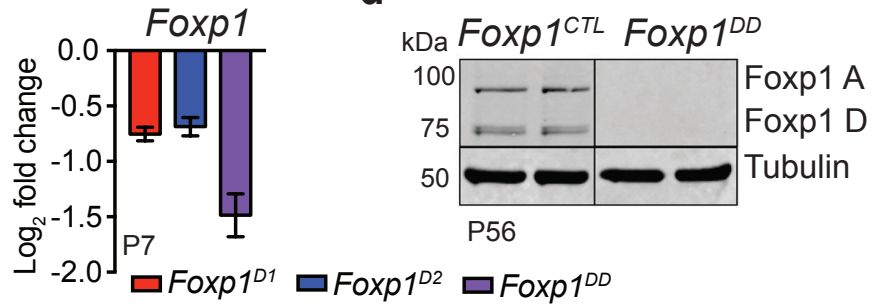

d

g

- Foxp1 $1^{\text {CTL }}$ cell $(14,466)$

h

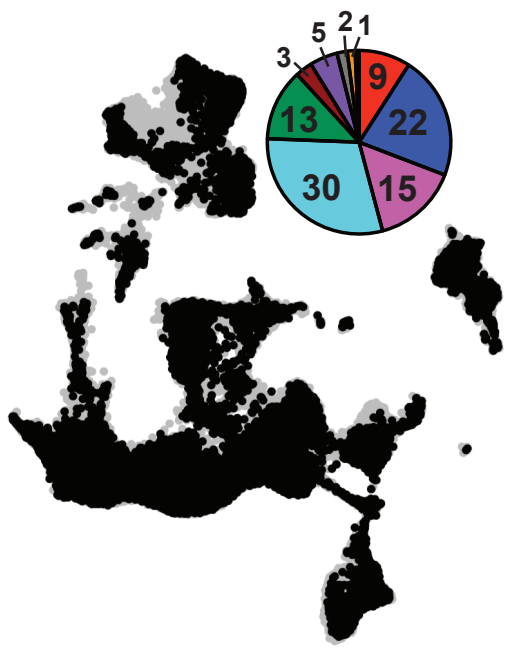

i - Foxp $1^{\text {D2 cell }}(9,898)$

j b

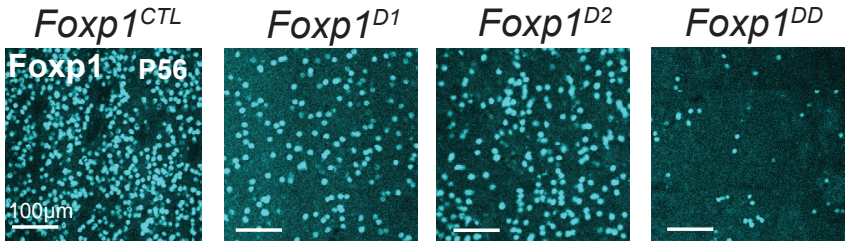
- Foxp1 $1^{D 1}$ cell $(16,961)$

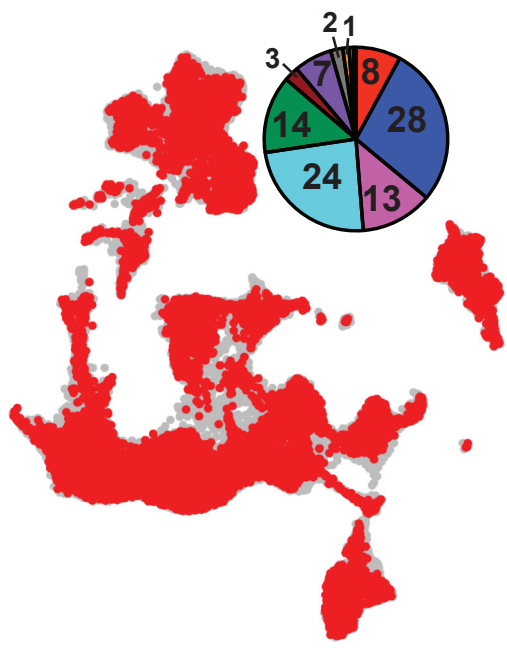

-Foxp $1^{D D}$ cell $(21,453)$
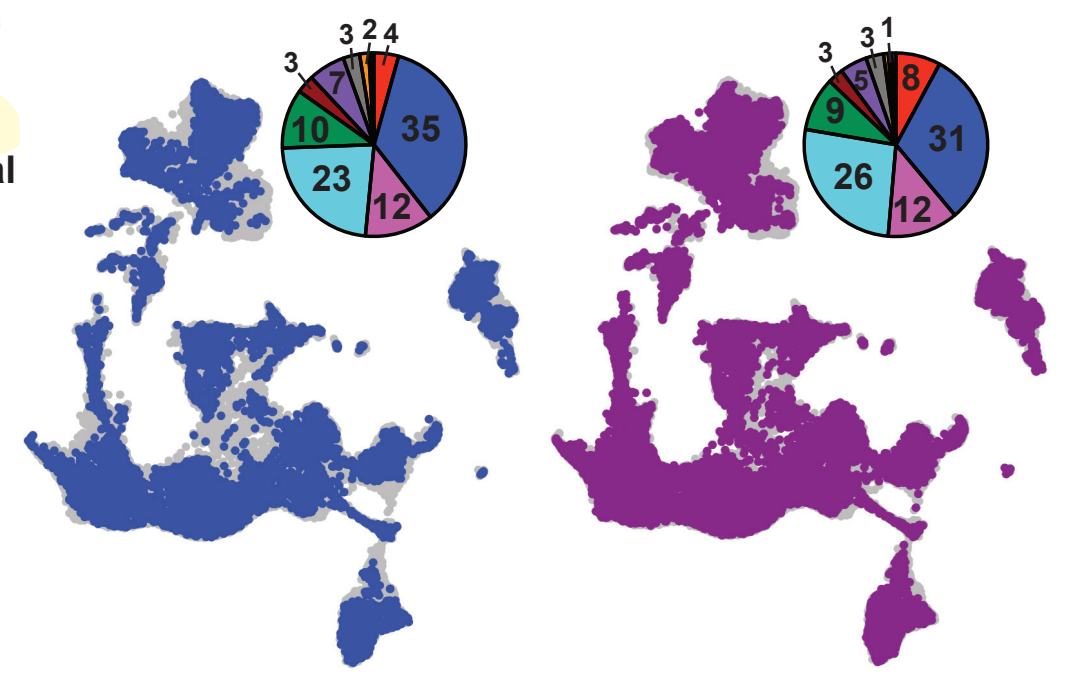
SupplemedipRxixprepxintegi:łttps://doi.org/10.1101/611780; this version posted April 18, 2019. The copyright holder for this preprint (which was not Supplemeftitfed by poetreview) is the author/funder, who has granted bioRxiv a license to display the preprint in perpetuity. It is made available under
aCC-BY-ND 4.0 International license.

a

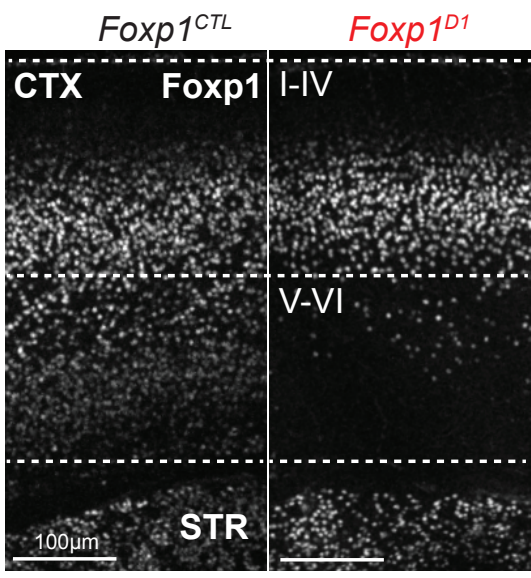

b

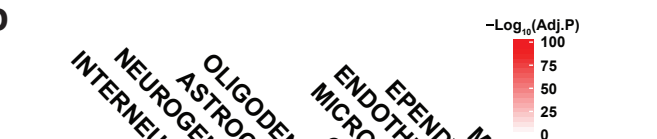

C

d
Aqp4

f

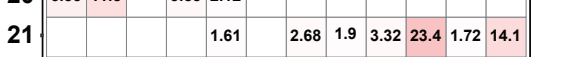

\begin{tabular}{|l|l|l|l|l|l|l|l|l|l|l|l|l|l|}
\hline 22 & 31.5 & 7.39 & 19.9 & 1.59 & & & & 1.9 & & & & & \\
\hline 23 & & & & & 42.8 & 2.13 & 5.52 & 2.71 & 43.7 & 18.2 & 8.01 & 6.99 \\
\hline
\end{tabular}

$24 \cdot 20.414 .59 .683 .26$

\begin{tabular}{l|l|l|l|l|l|l|}
\hline 25 & 1.49 & 3.2 & & 1.78 & 1.95 \\
\hline
\end{tabular}

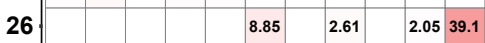

$27 \cdot 71.217 .158 .52 .62$

$28 \cdot 23.5 \quad 10.5 \quad 11.52 .63$

$29 \cdot$\begin{tabular}{ll|l|l|l|l|l}
14.7 & 6.51 & 4.61 & 6
\end{tabular}

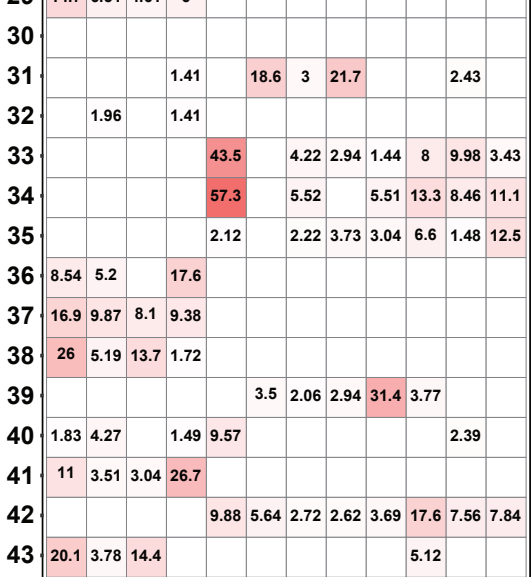
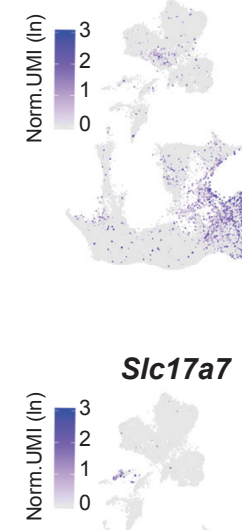

e
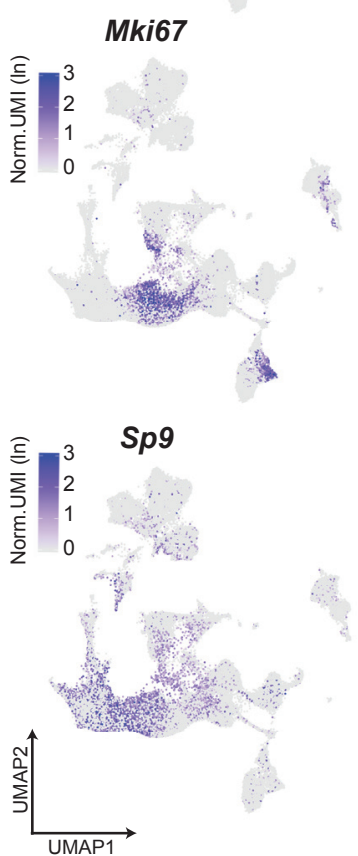

g

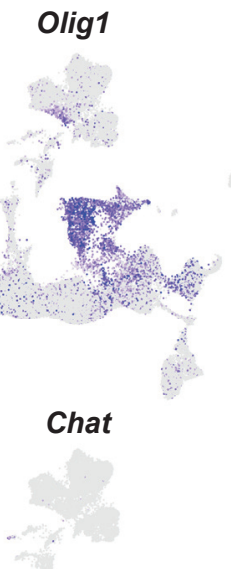

Ascl1

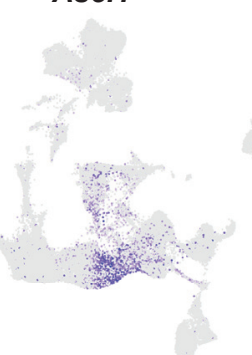

Ppp1r1b
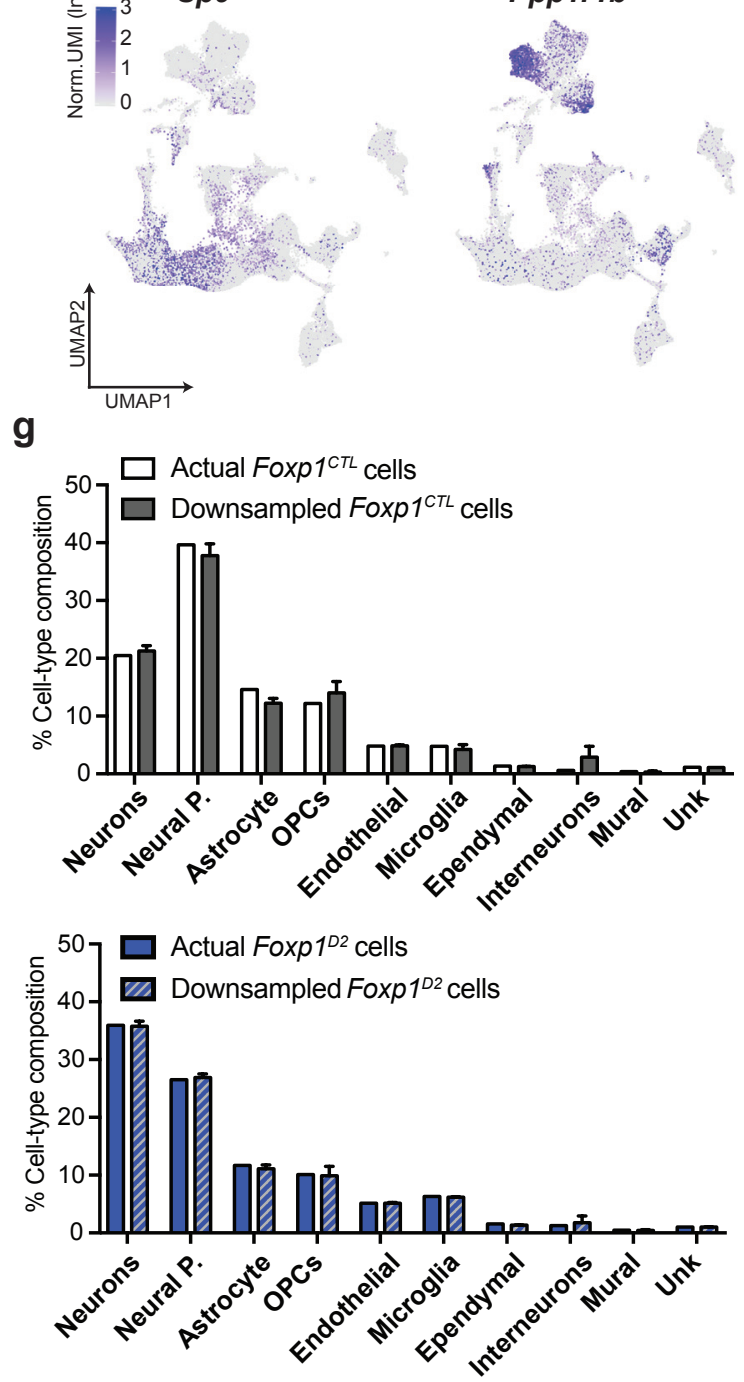

Flt1

Cx3cr1
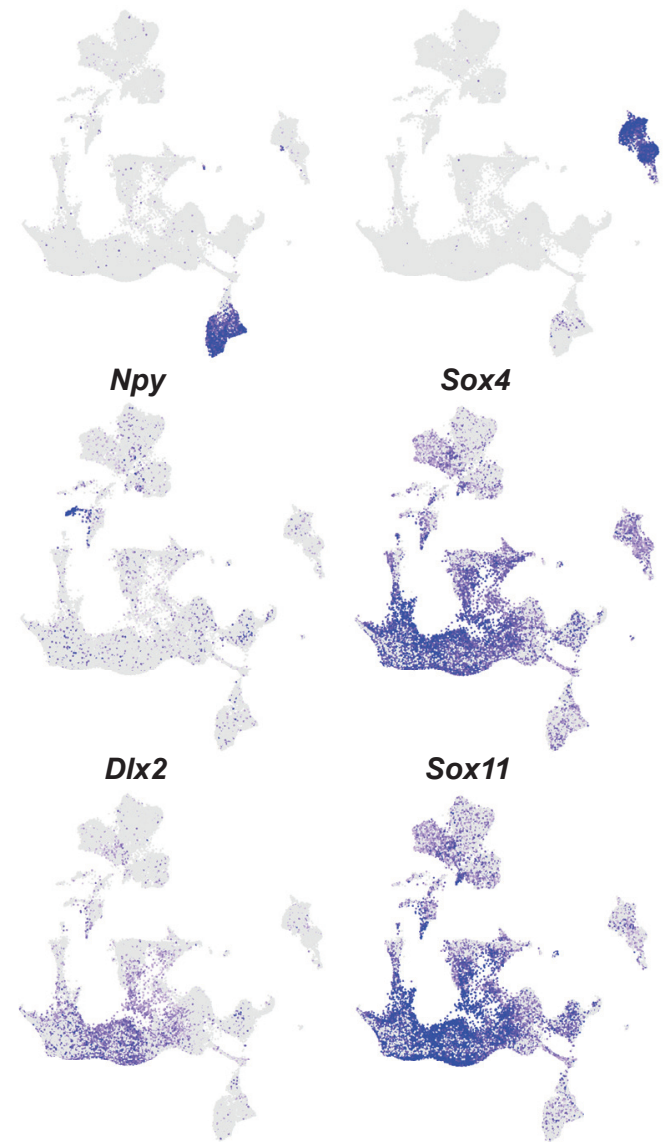

Drd1

Drd2
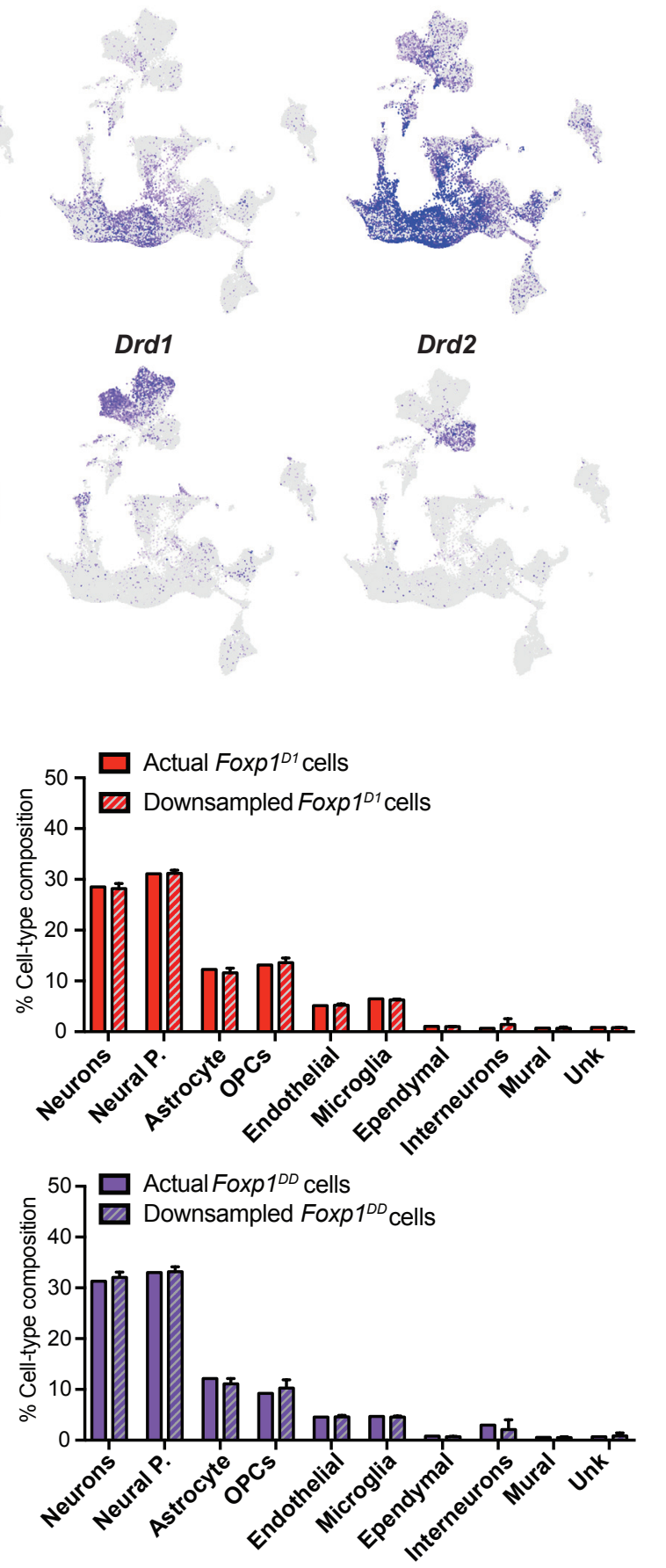
Fig 2 bioRxiv preprint doi: https://doi.org/10.1101/611780; this version posted April 18, 2019. The copyright holder for this preprint (which was not 1 certified by peer review) is the author/funder, who has granted bioRxiv a license to display the preprint in perpetuity. It is made available under

a

\section{8,073 neurons} Nkx2-1+

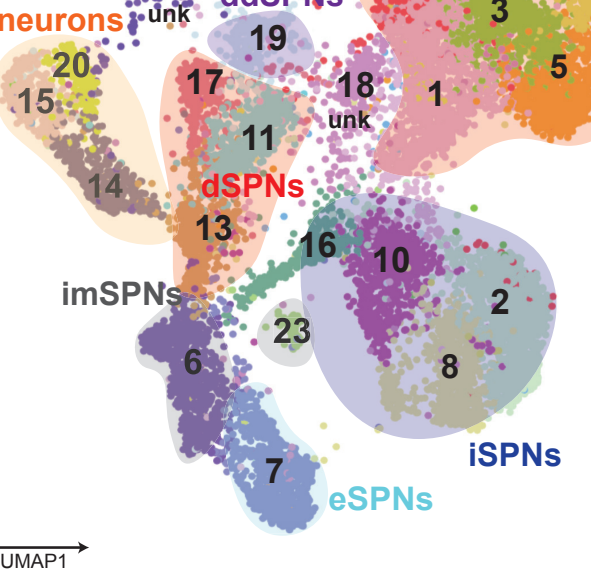

\section{dSPNs}

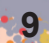

4

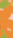
$\mathrm{aCC}_{\mathbf{f}} \mathrm{BY}-\mathrm{ND} 4.0$ International license.

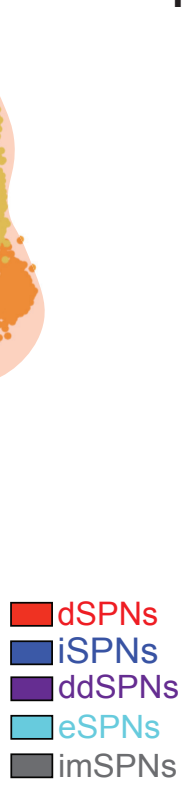

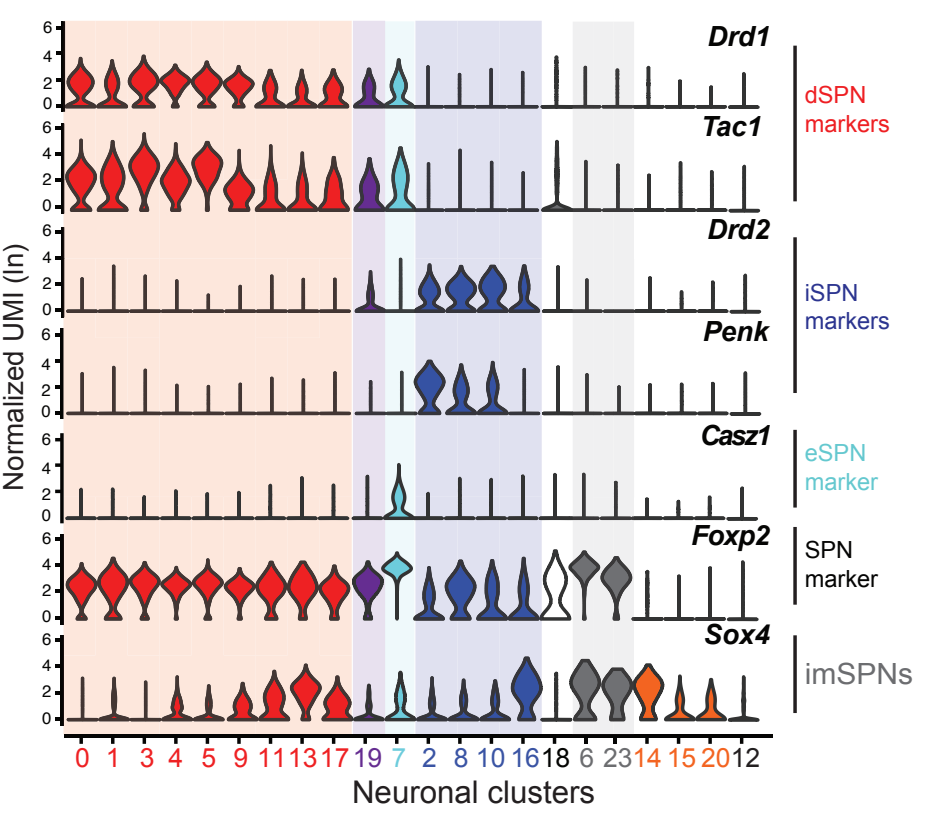

b

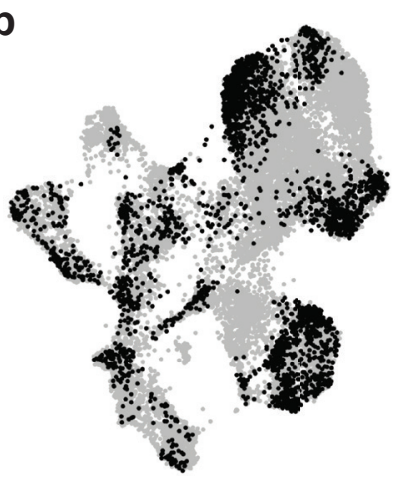

- Foxp $1^{\text {CTL }}$ cell

d

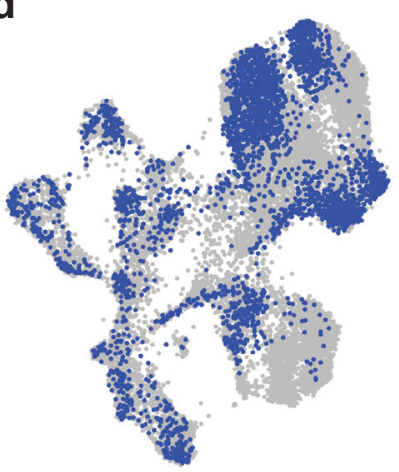

- Foxp $1^{D 2}$ cell
C

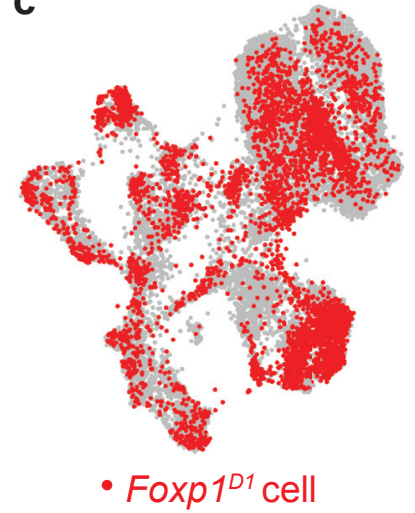

e

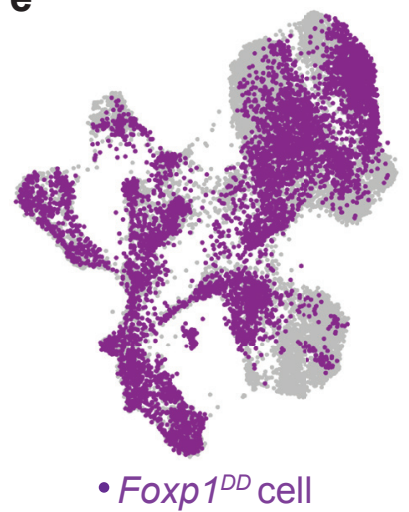

g

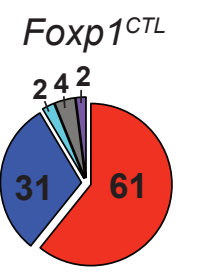

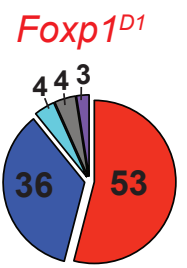

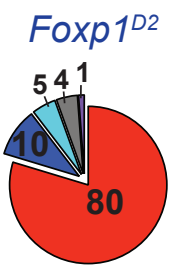

Foxp $1^{D D}$

h
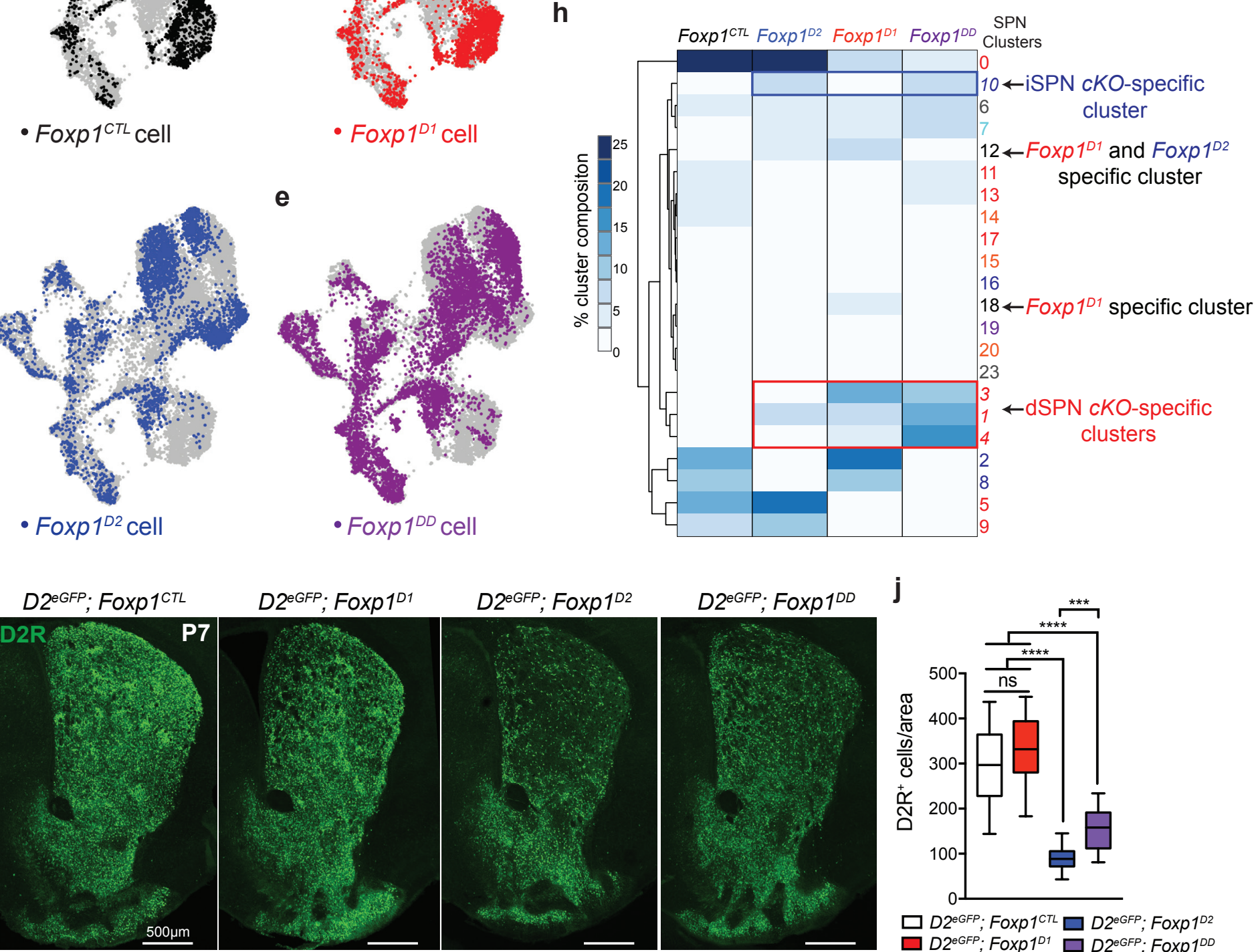

$D 2^{\text {EGFP }} ;$ Foxp $1^{D 1}$

$D 2^{\mathrm{EGFP}} ;$ Foxp1 ${ }^{D 2}$

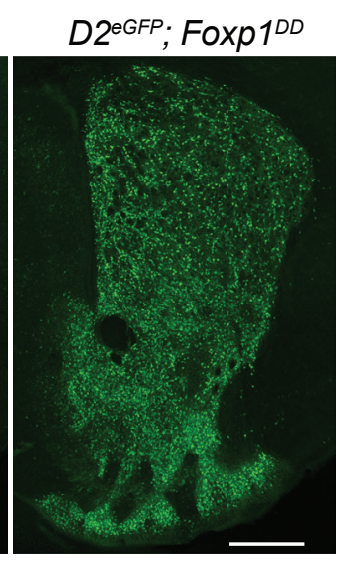

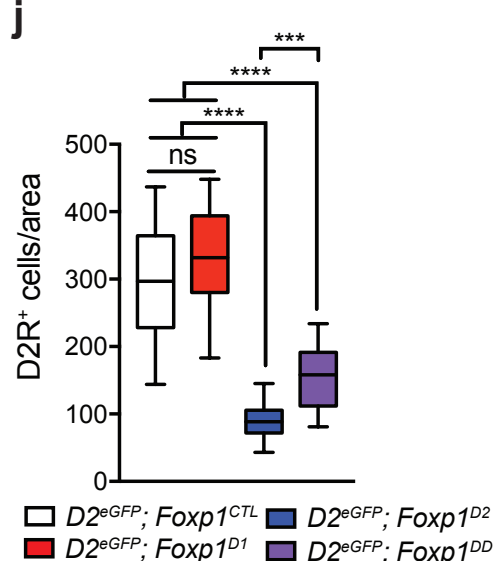

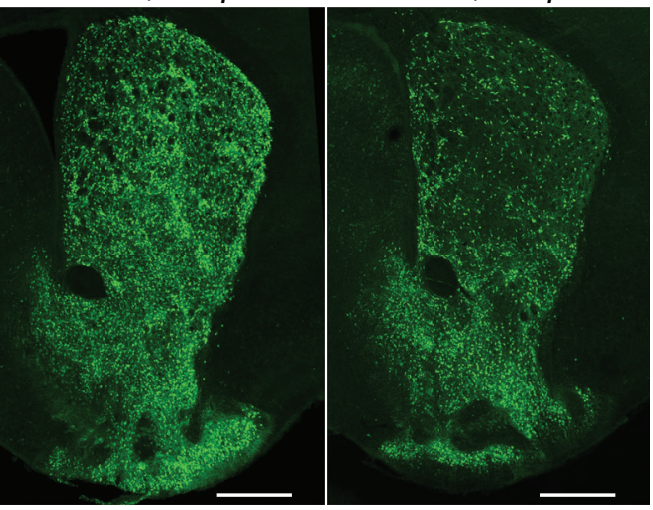

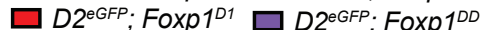




\section{Nkx2-1 Drd1}

I

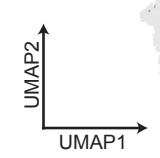

d

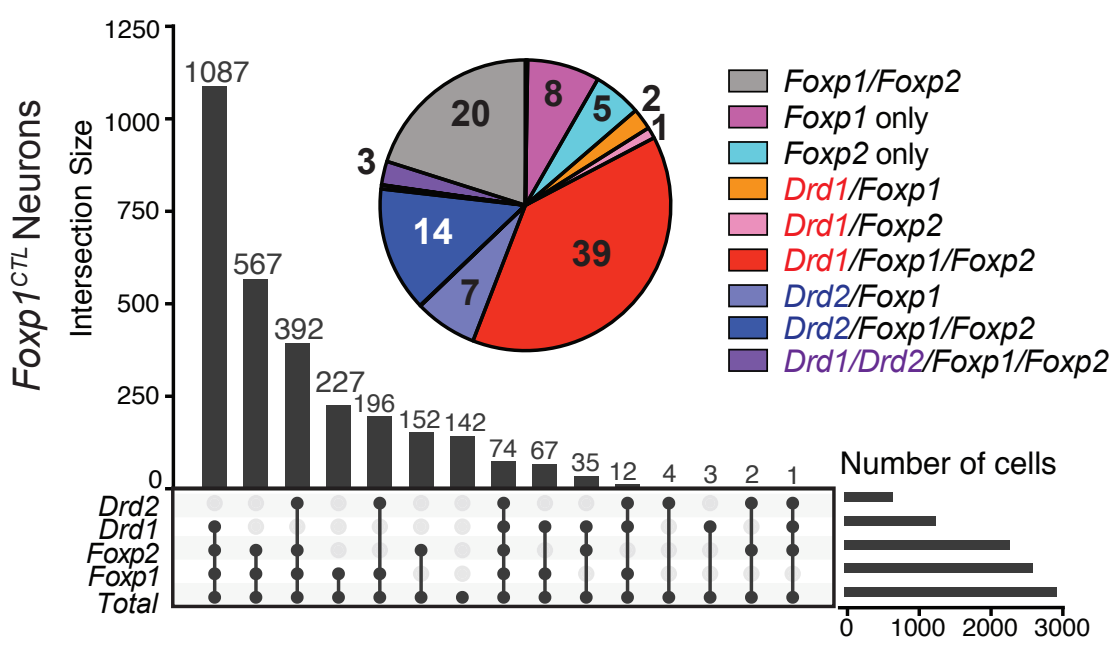

g

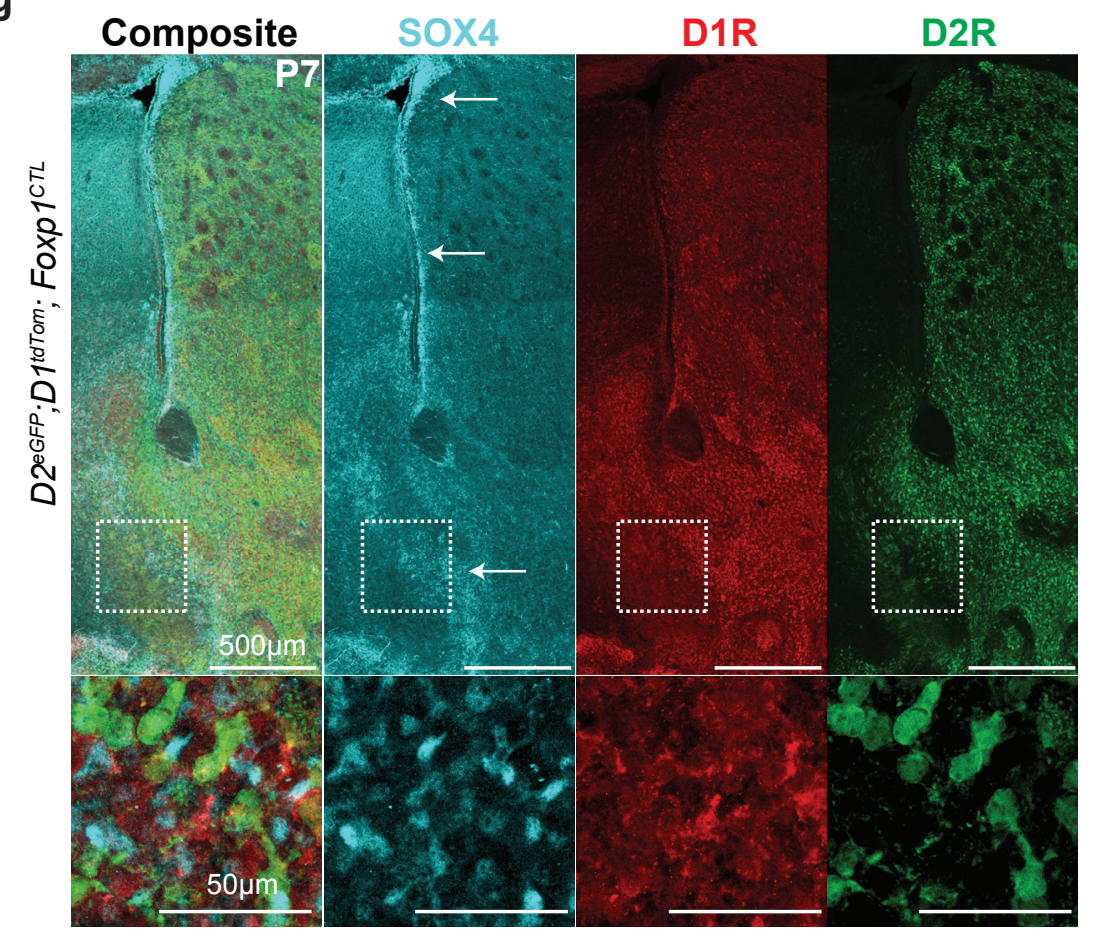

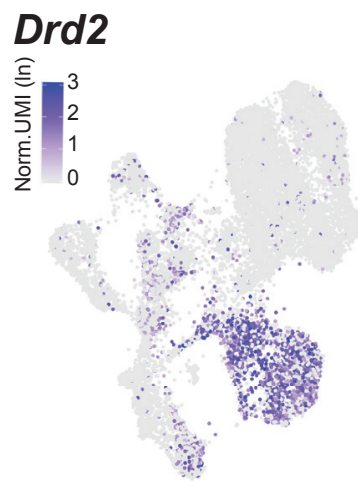

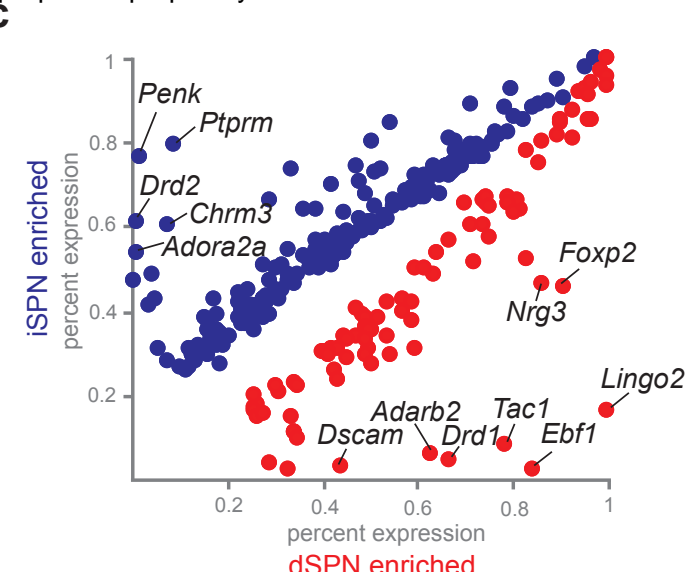

e

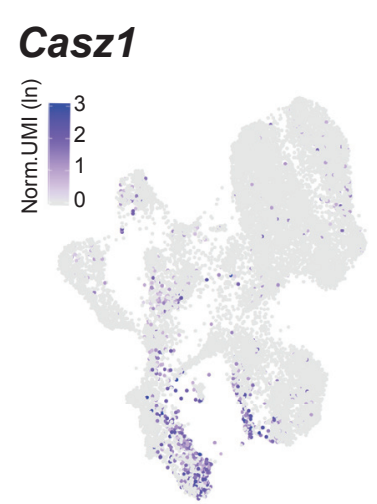

f

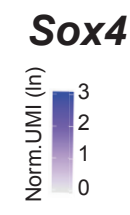

h

Foxp2

ll:
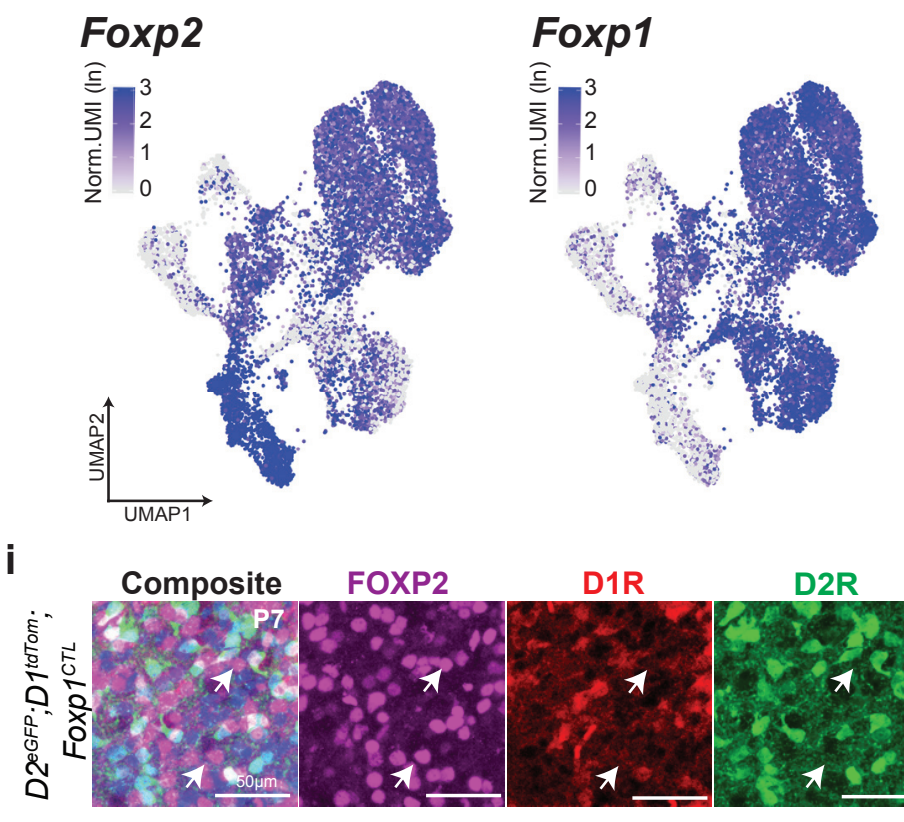

j
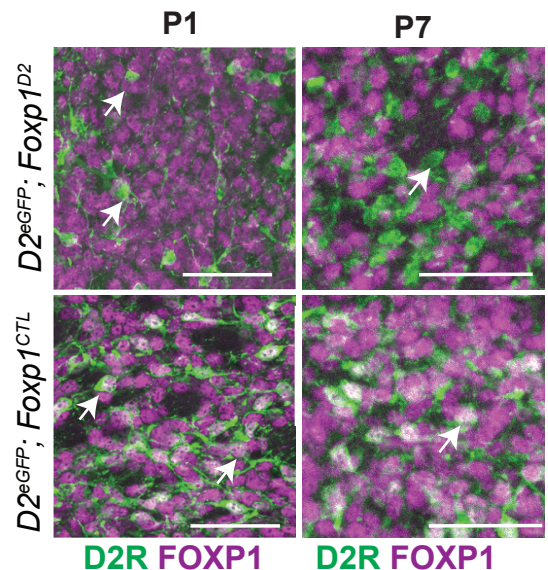

D1R

D2R

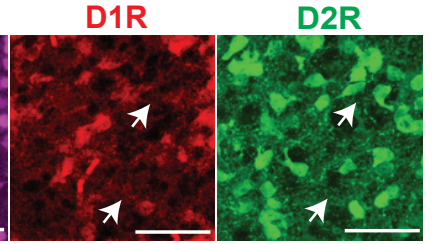

P56

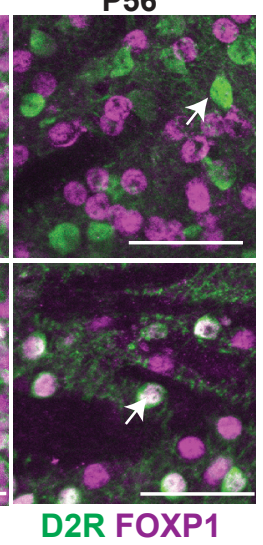


bioRxiv preprint doi: https://doi.org/10.1101/611780; this version posted April 18,2019 . The copyright holder for this preprint (which was not certified by peer review) is the author/funder, who has granted bioRxiv a license to display the preprint in perpetuity. It is made available under aCC-BY-ND 4.0 International license.

a

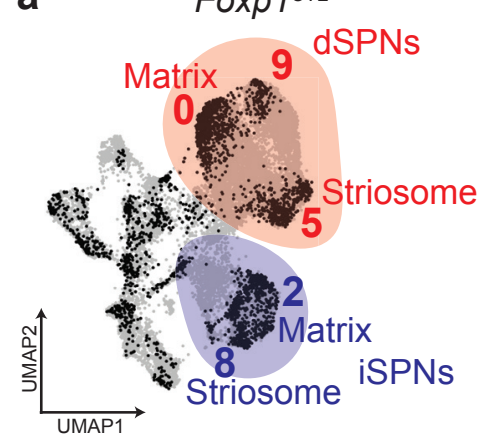

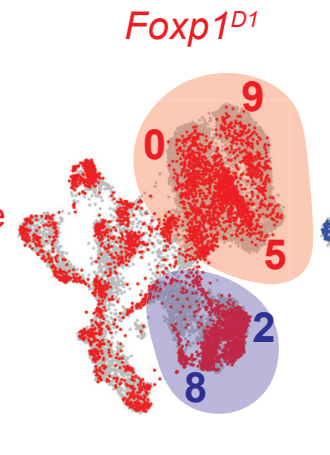

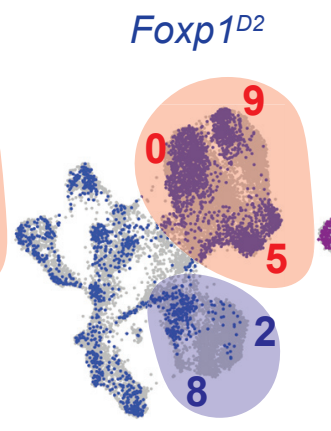

d

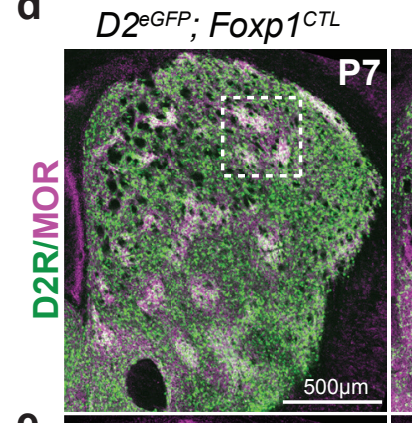

$D 2^{\mathrm{eGFP}} ;$ Foxp $1^{D 1}$
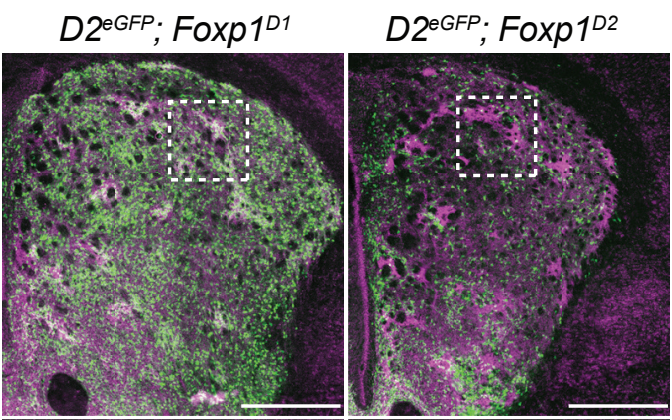

e
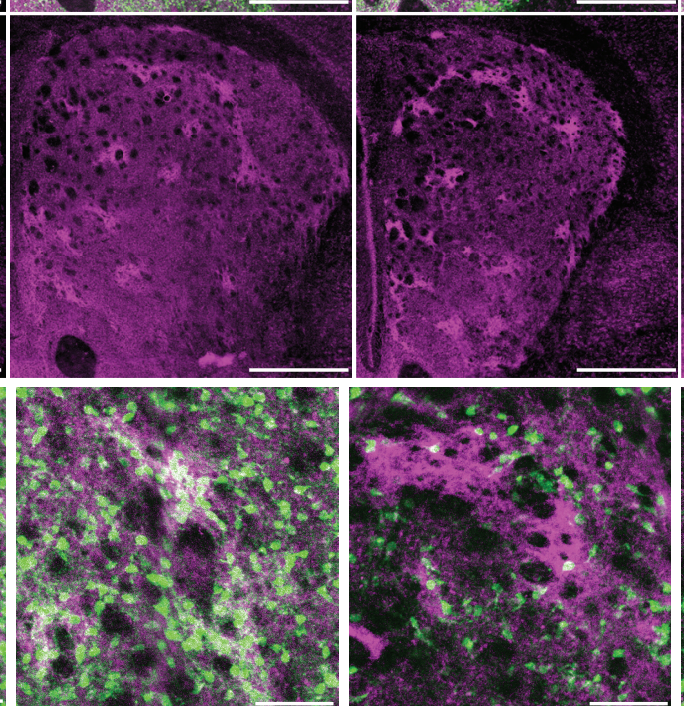

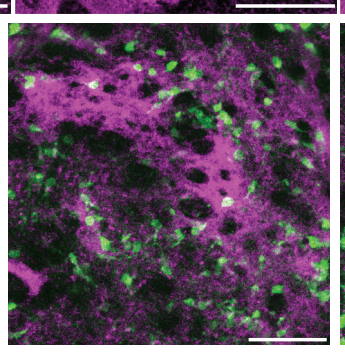

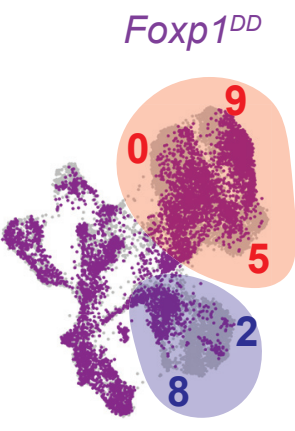

$D 2^{\mathrm{eGFP}} ;$ Foxp $1^{D D}$

b

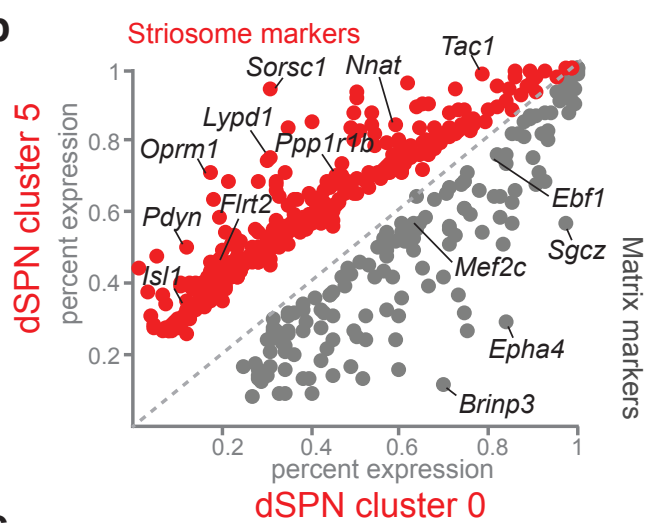

C
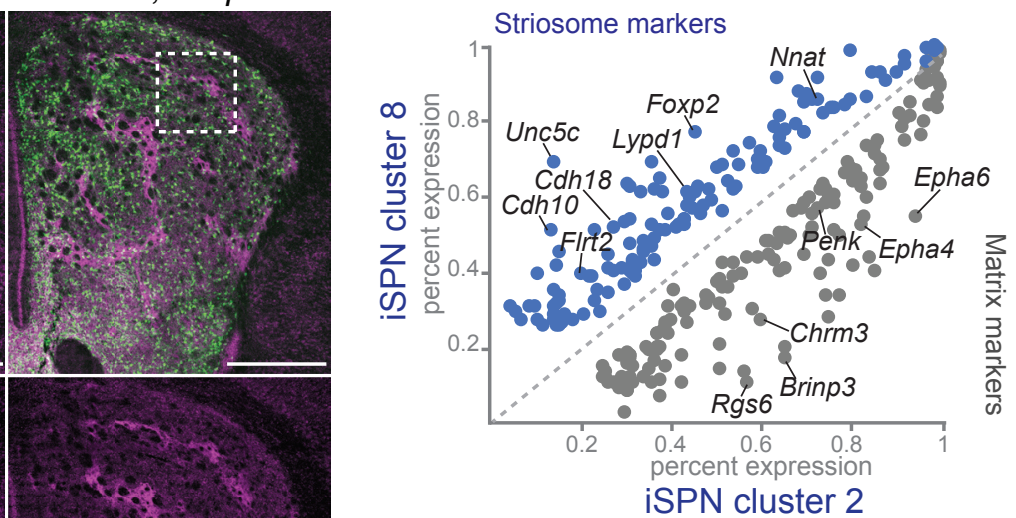

$\mathrm{g}$ h

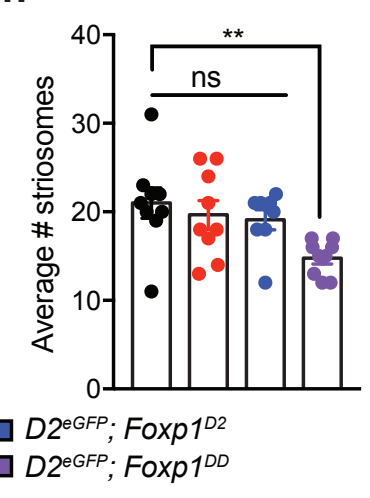


Fig 4 bioRxiv preprint doi: https://doi.org/10.1101/611780; this version posted April 18, 2019. The copyright holder for this preprint (which was not certified by peer review) is the author/funder, who has granted bioRxiv a license to display the preprint in perpetuity. It is made available under aCC-BY-ND 4.0 International license.

a

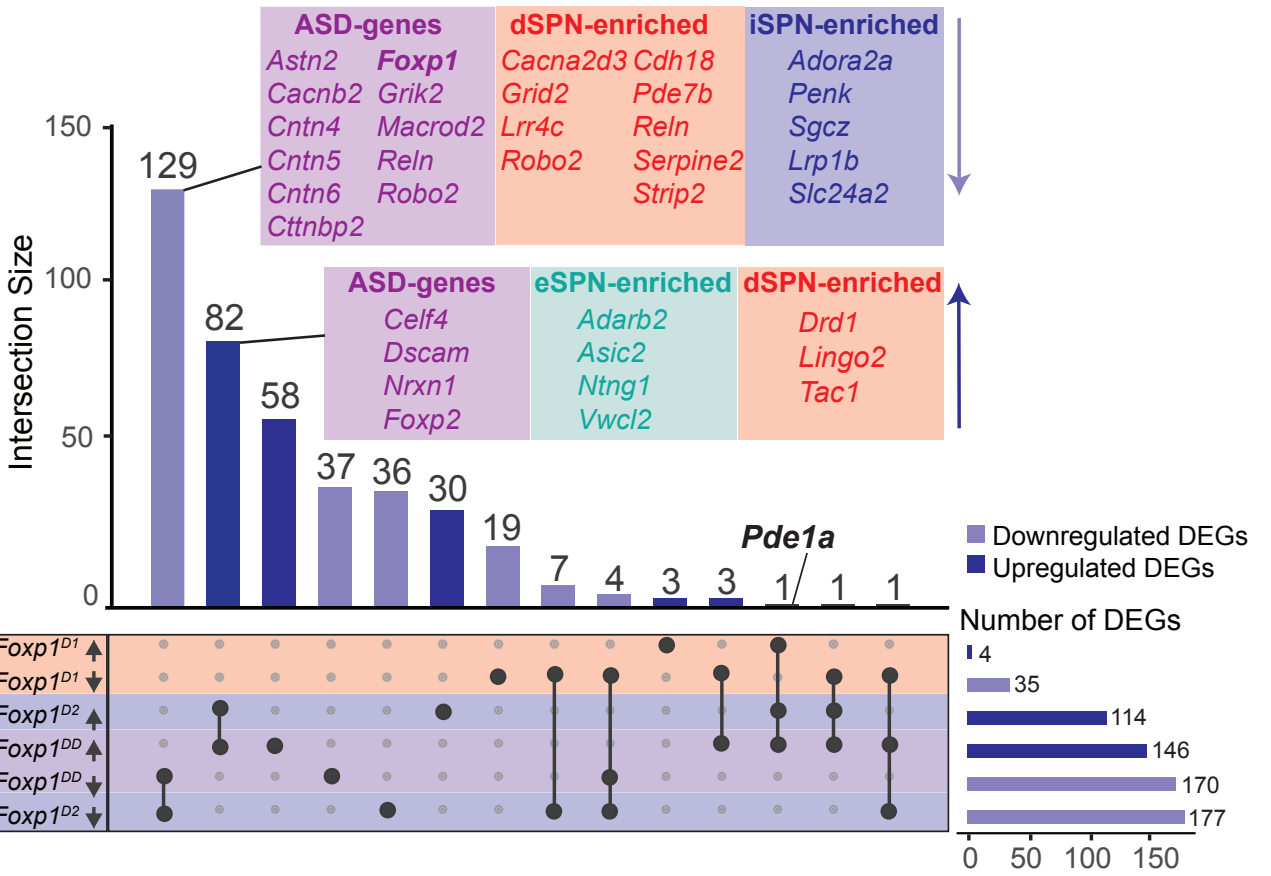

b
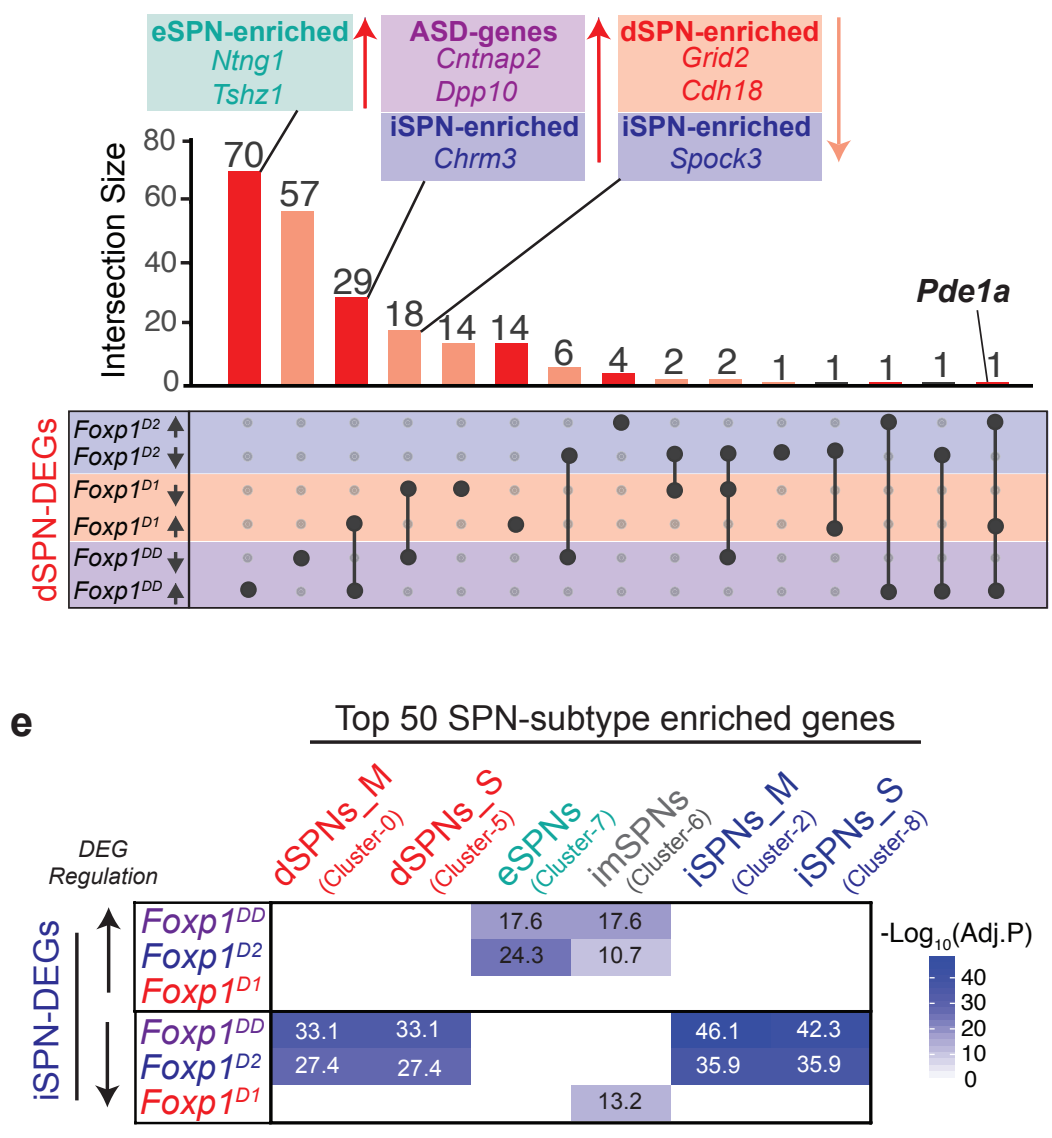

f
C

$\square$ Non-cell-autonomous DEGs

$\square$ Cell-autonomous Foxp $1^{D 2}$ DEGs

$\square$ Cell-autonomous Foxp $1^{D 1}$ DEGs

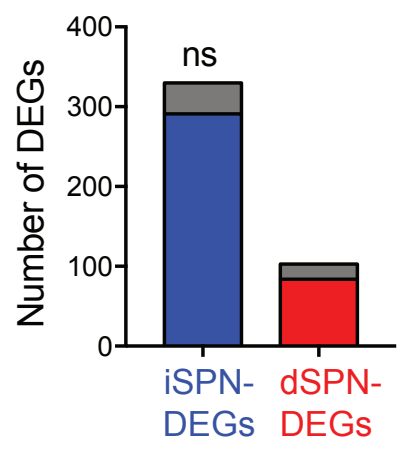

d

Downregulated DEGs

- Upregulated DEGs

Number of DEGs

$=6$

13

36

45
$\square$

$\begin{array}{lllll}0 & 25 & 50 & 7 \dot{5} & 1020\end{array}$
Interaction: Foxp $1^{D D}$ only DEGs

Shared with Foxp $1^{D 2}$ DEGs

$\square$ Shared with Foxp $1^{D 1}$ DEGs

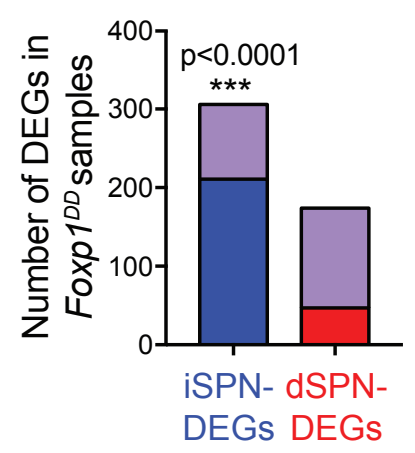

Top 50 SPN-subtype enriched genes

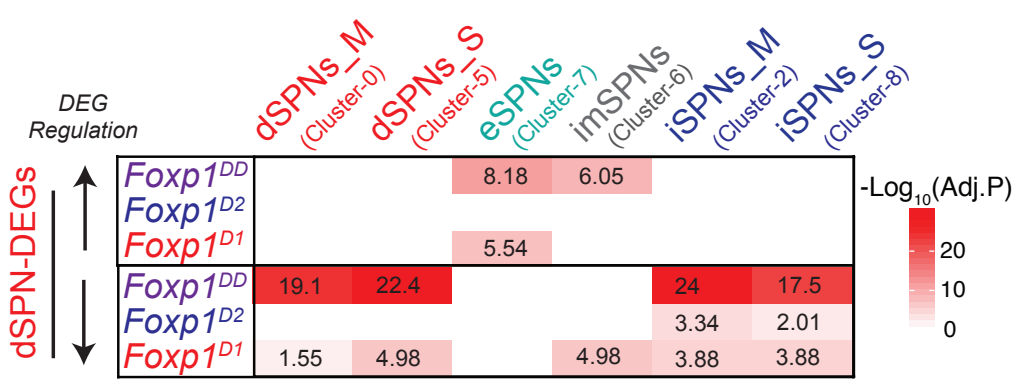


SubolemepiథBxiv queprintroizhttps://doi.org/10.1101/611780; this version posted April 18, 2019. The copyright holder for this preprint (which was not

a

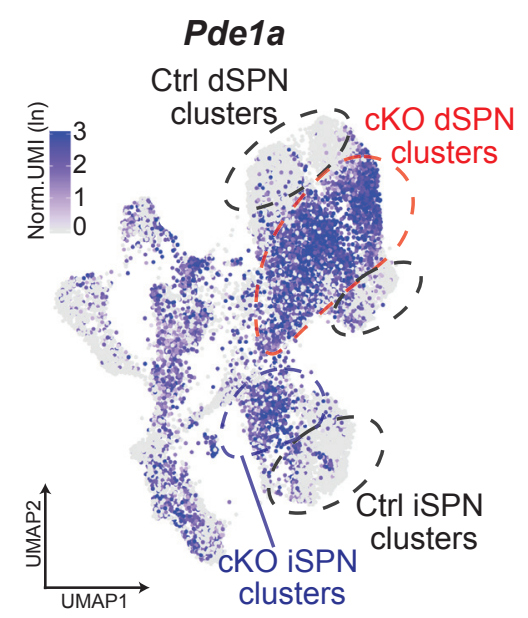

b

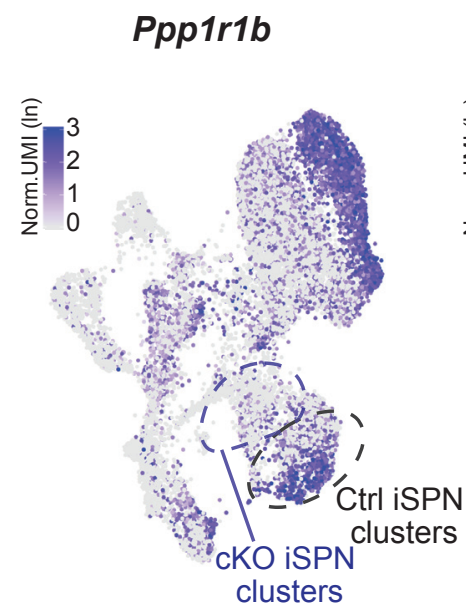
4.0 International license.

C

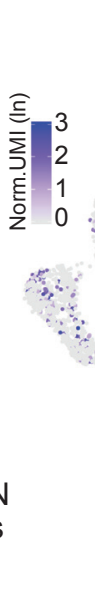

\section{Calb1}

Ctrl dSPN

clusters, - cKO dSPN

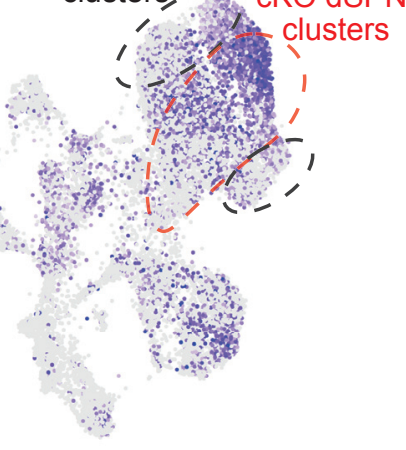

d
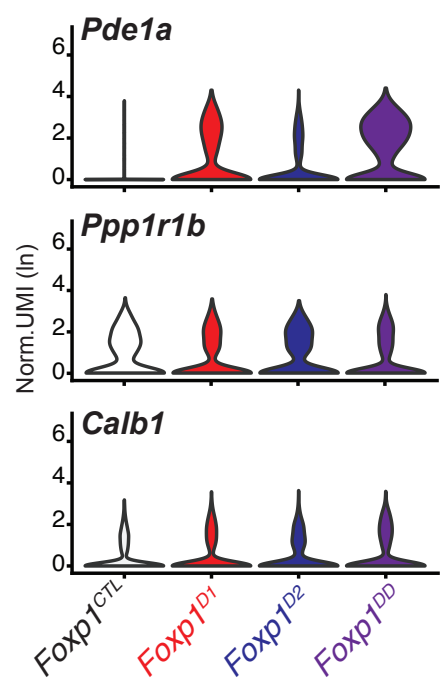

e

\begin{tabular}{cccc}
\multicolumn{4}{c}{ Foxp1 $^{\text {CTL }}$} \\
\hline DEG Target & D1R & D2R & Combined
\end{tabular}

\begin{tabular}{lrrr}
\multicolumn{4}{c}{ Foxp1 $^{D D}$} \\
\hline DEG Target & D1R & D2R & Combined
\end{tabular}
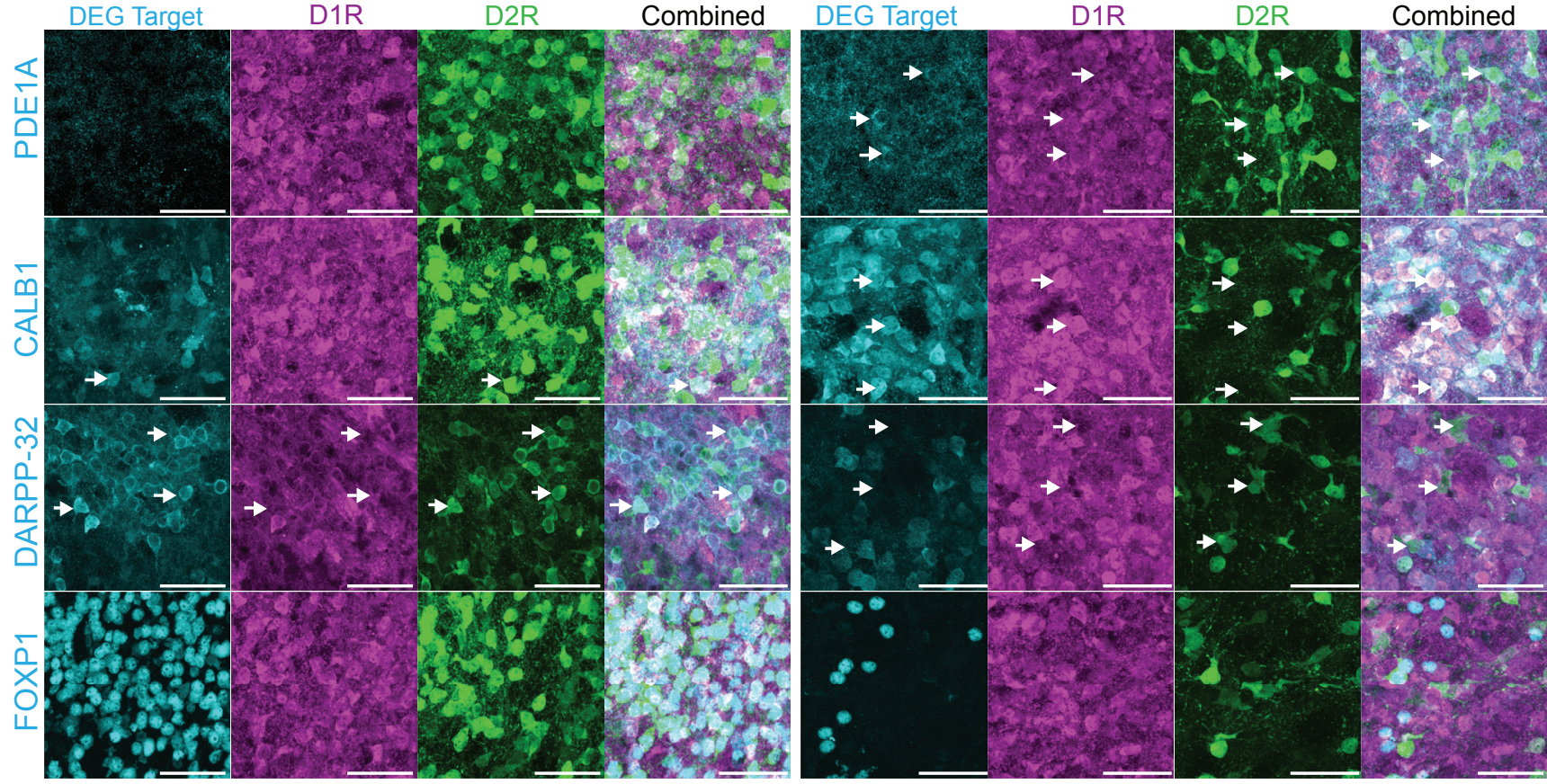

dSPNs

iSPNs
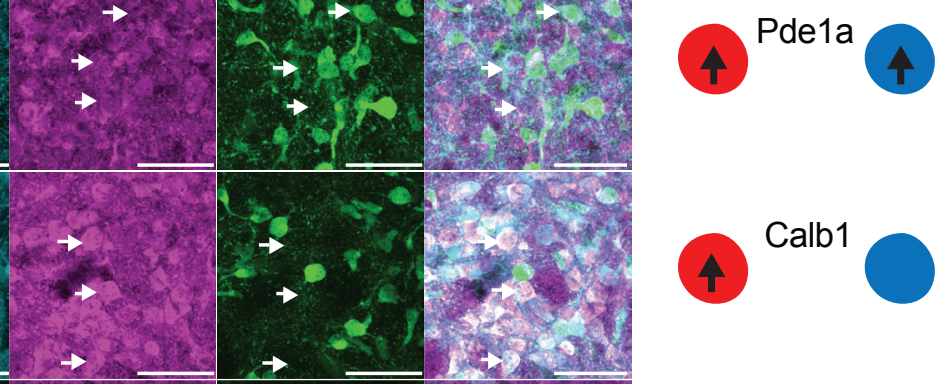

(4)

Calb1
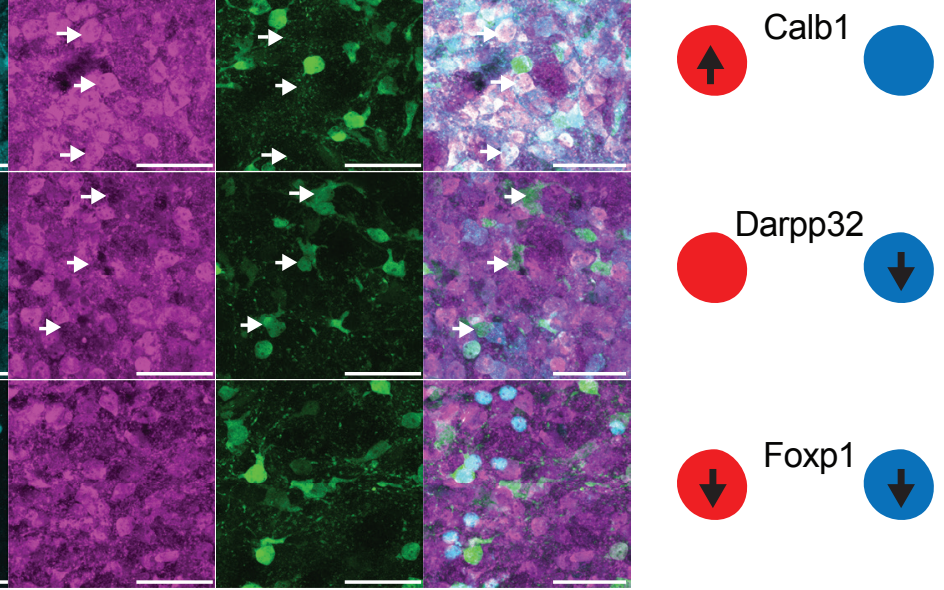

Foxp1

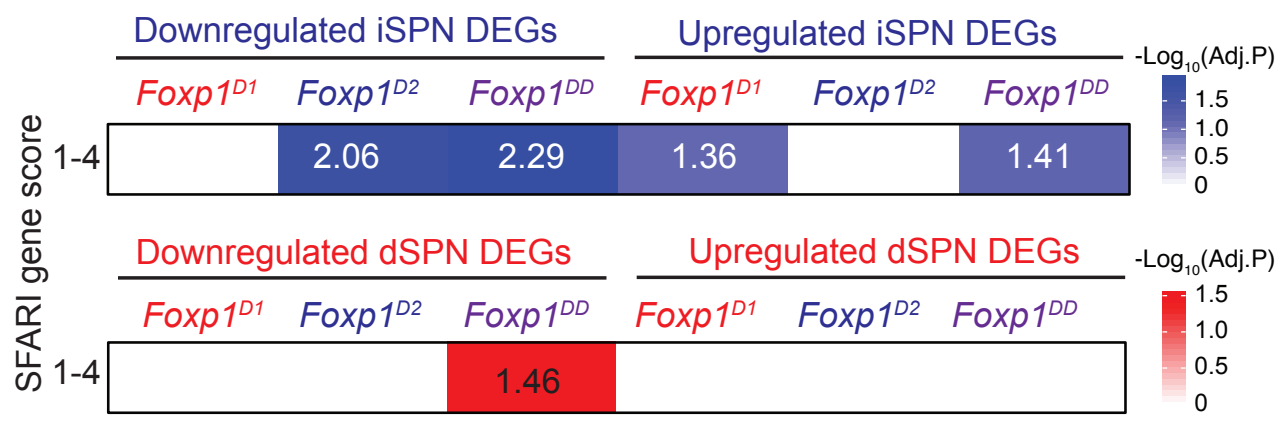


Finure 5 bioRxiv preprint doi: https://doi.org/10.1101/611780; this version posted April 18, 2019. The copyright holder for this preprint (which was not Figure 5 certified by peer review) is the author/funder, who has granted bioRxiv a license to display the preprint in perpetuity. It is made available under

a

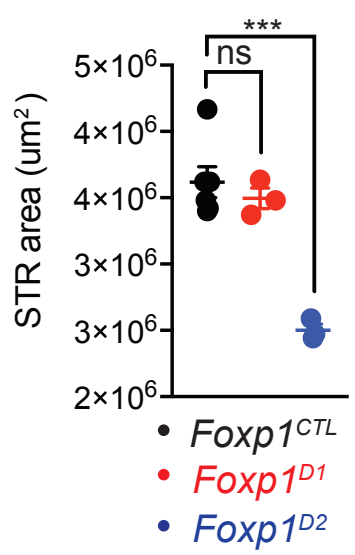
aCC-BY-ND 4.0 International license.

b $D 2^{\text {EGFP }} ; D 1^{\text {ddTom }} ;$ Foxp1 ${ }^{\text {CTL }}$
C

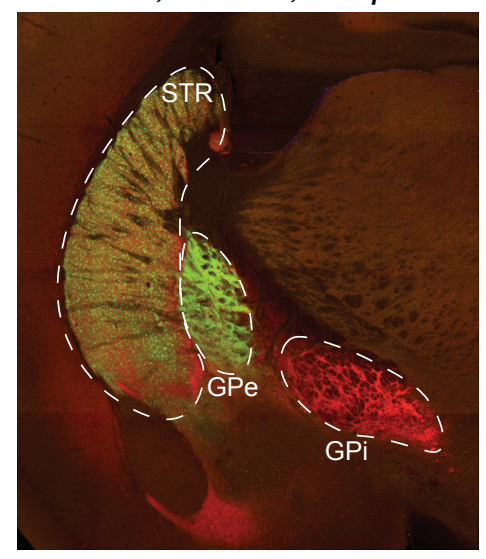

e

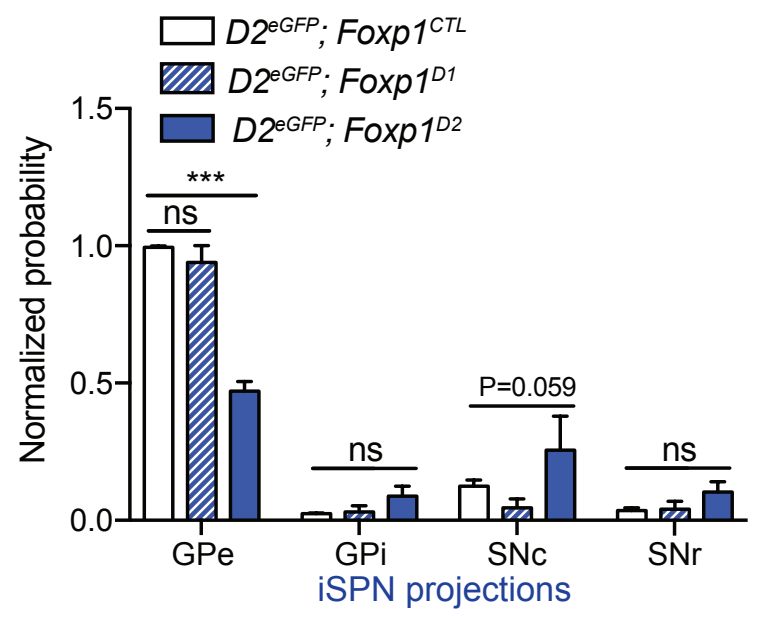

g
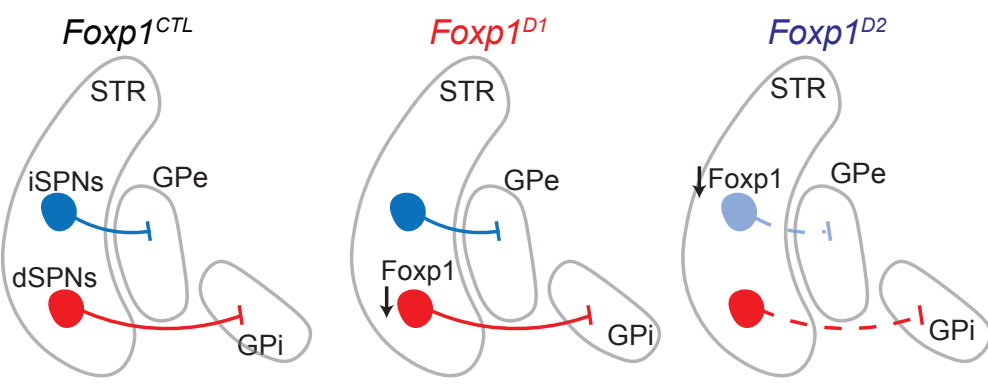

iSPN projections dSPN projections

f

\section{d}

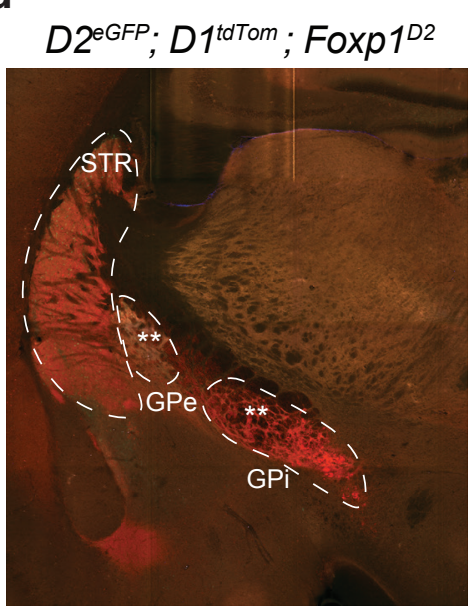

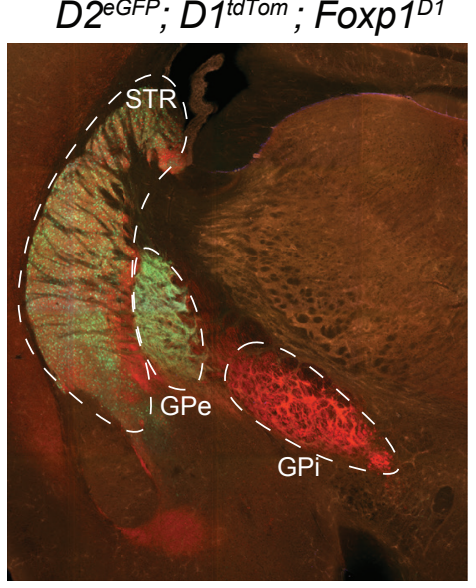

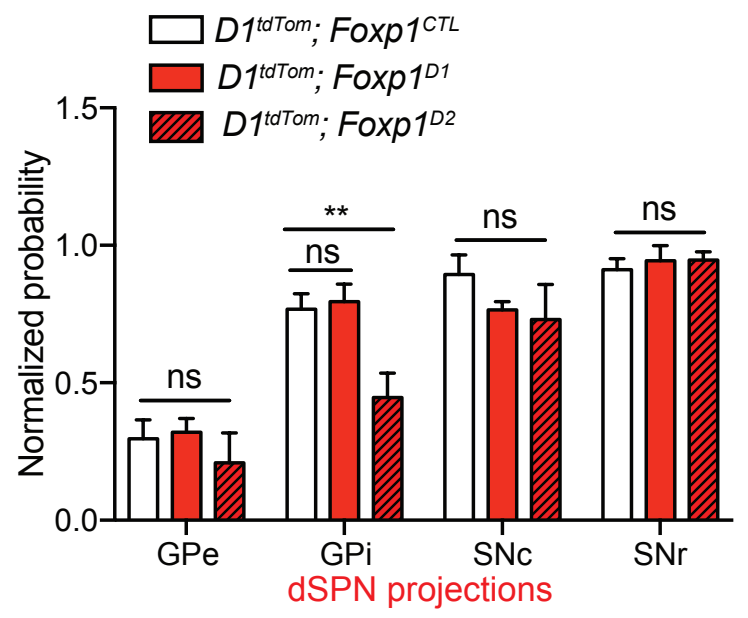

h dSPN-DEGs

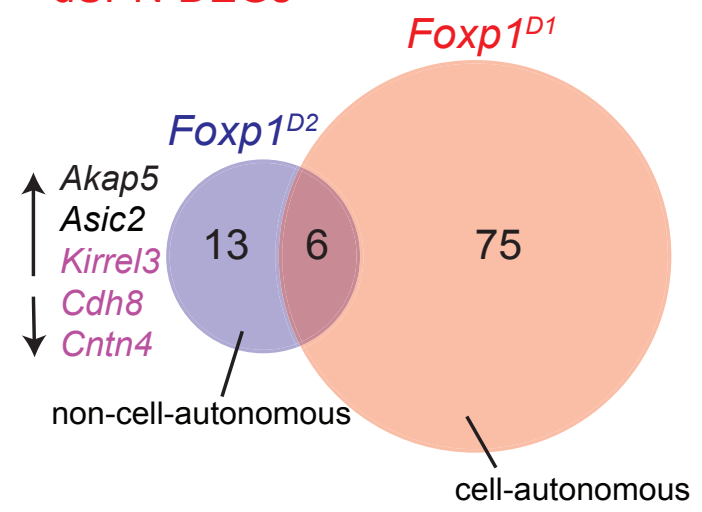


bioRxiv preprint doi: https://doi.org/10.1101/611780; this version posted April 18, 2019. The copyright holder for this preprint (which was not Fig. 6 certified by peer review) is the author/funder, who has granted bioRxiv a license to display the preprint in perpetuity. It is made available under

a

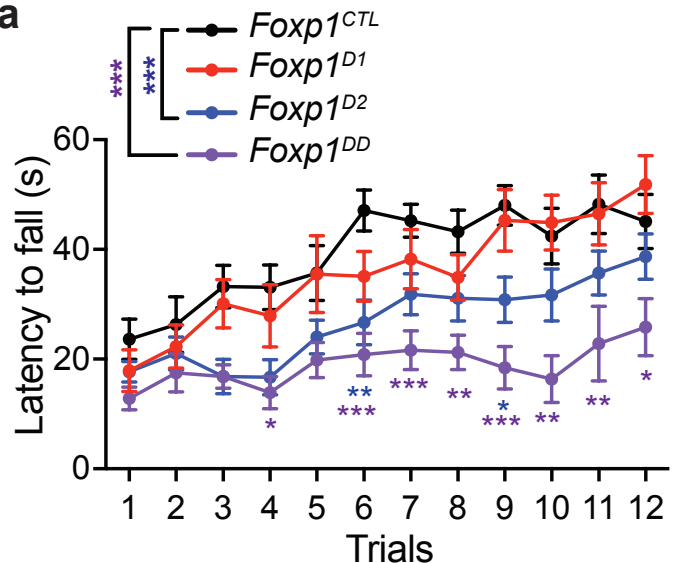

b
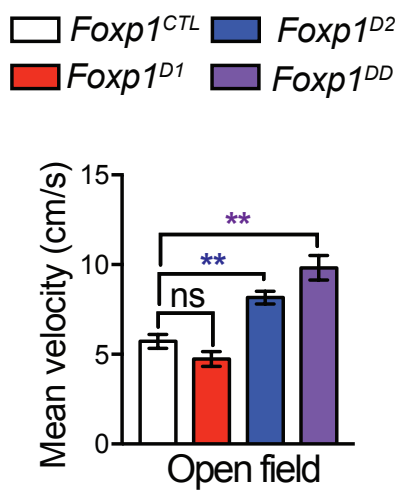

e

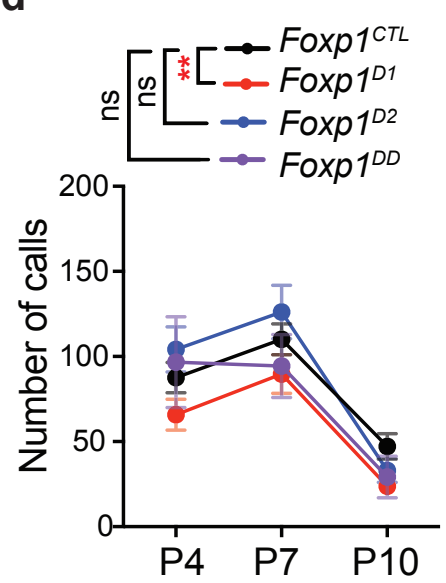

g
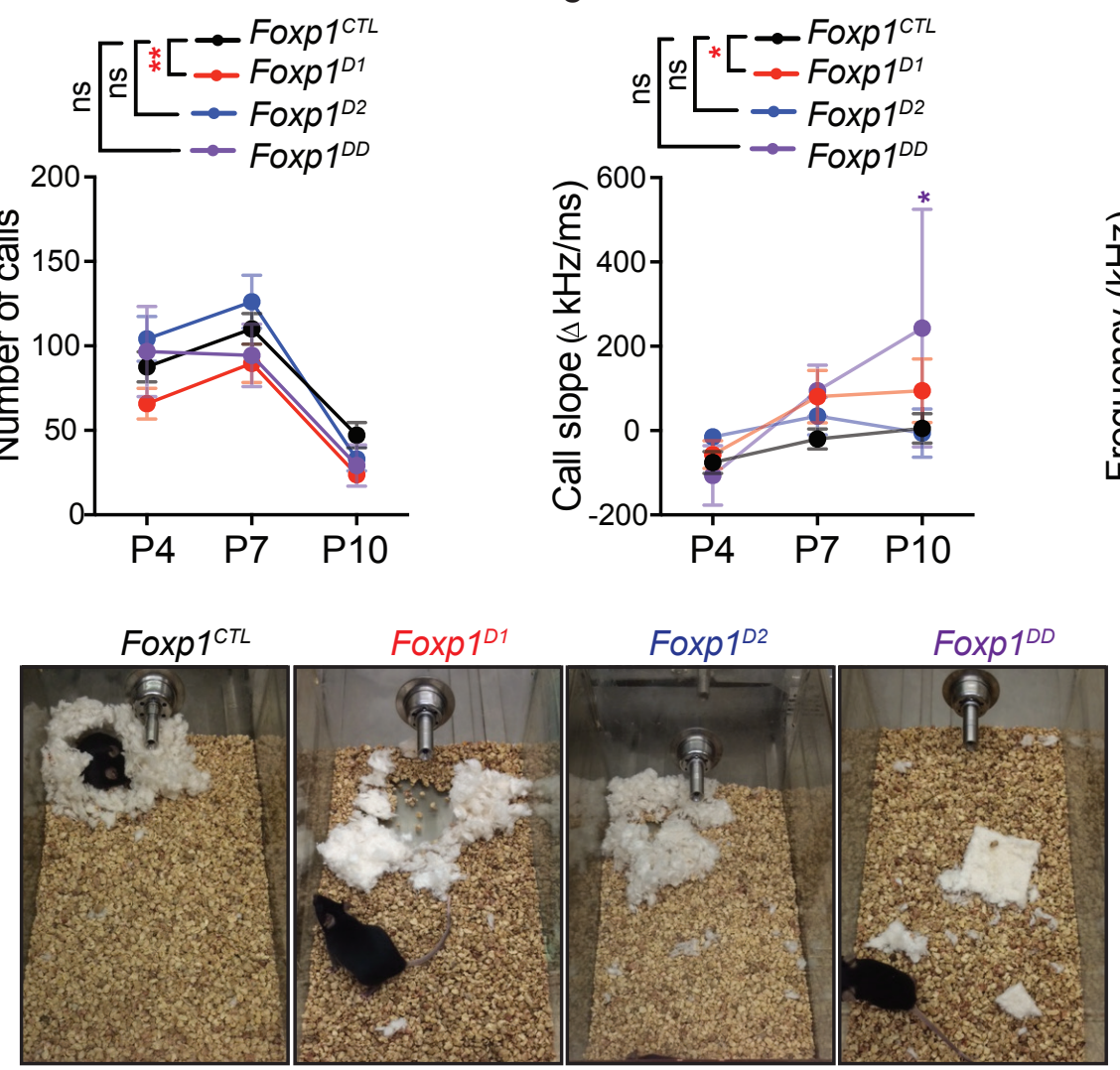

c
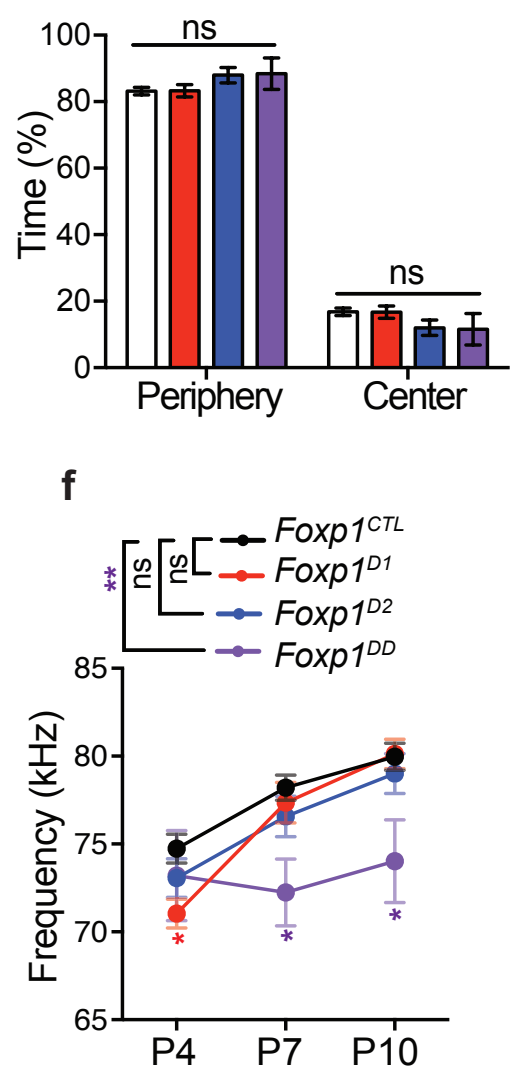

h

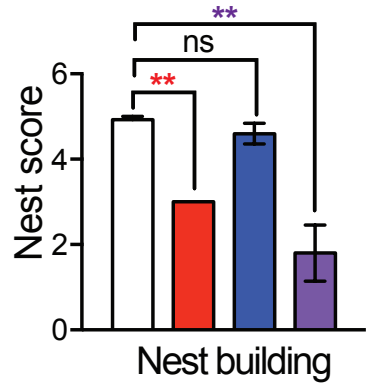

i

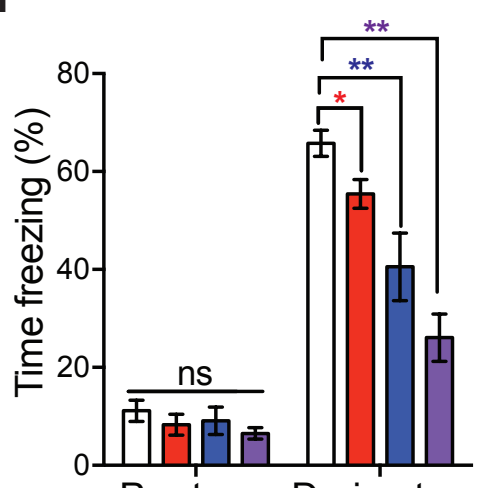

Pre-tone During tone Cued FC

j

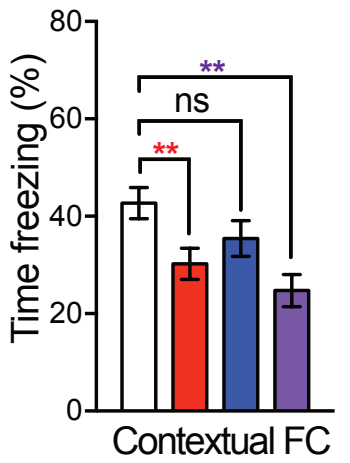


bioRxiv preprint doi: https://doi.org/10.1101/611780; this version posted April 18, 2019. The copyright holder for this preprint (which was not certified by peer review) is the author/funder, who has granted bioRxiv a license to display the preprint in perpetuity. It is made available under

\section{Foxp1 ${ }^{\text {CTL }}$} aCC-BY-ND 4.0 International license.

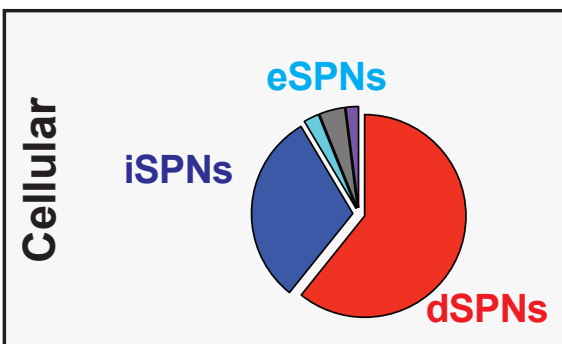

Foxp1D1

Foxp1 ${ }^{D 2}$
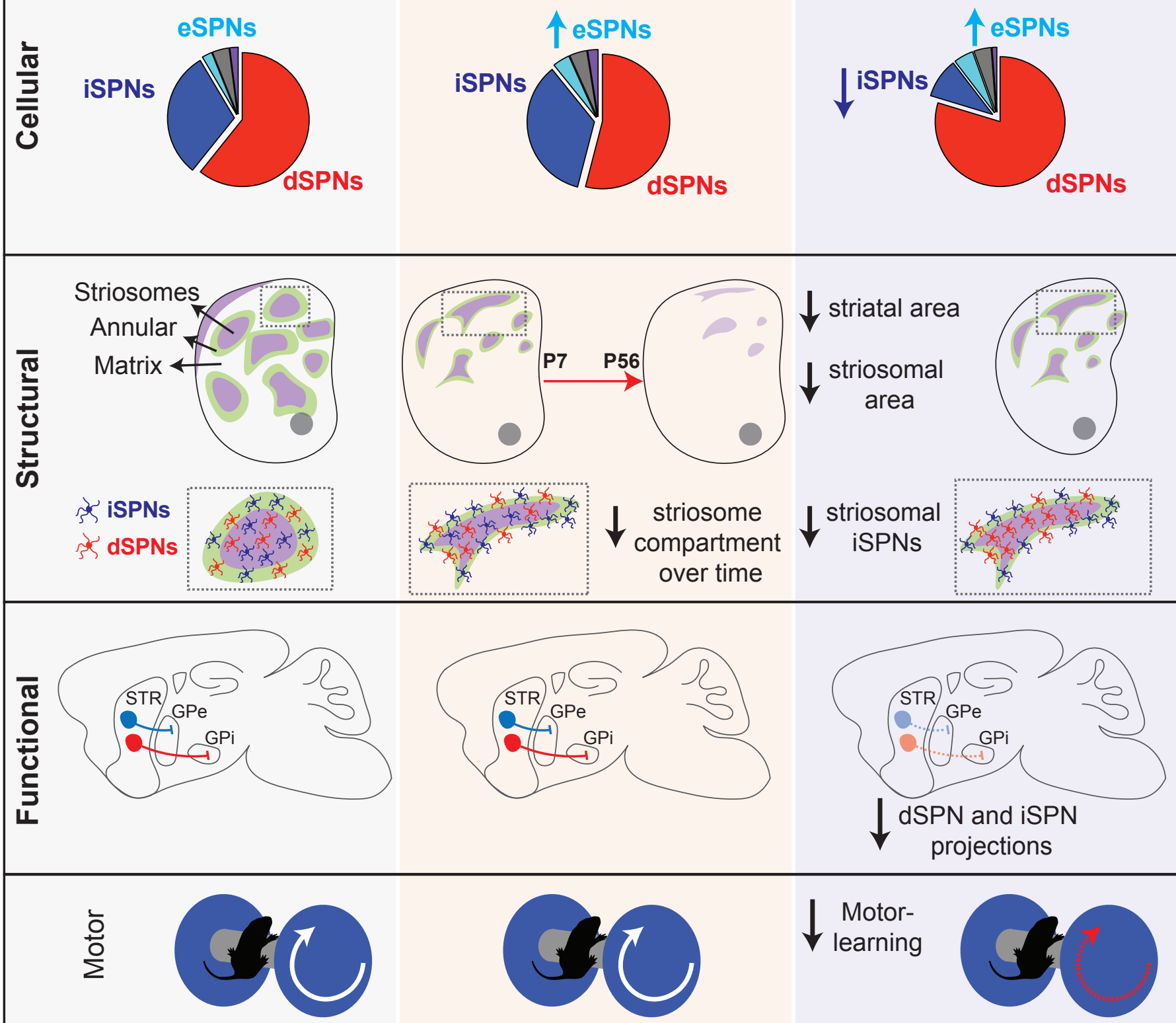

$\frac{\bar{\pi}}{\frac{\pi}{2}}$
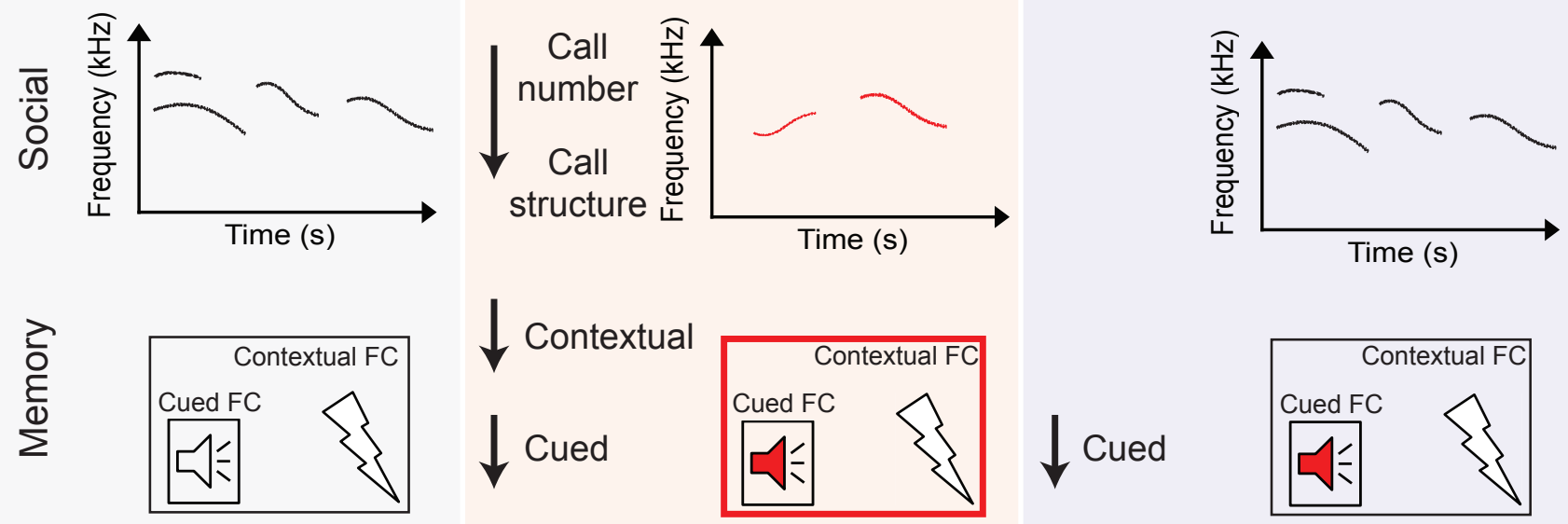